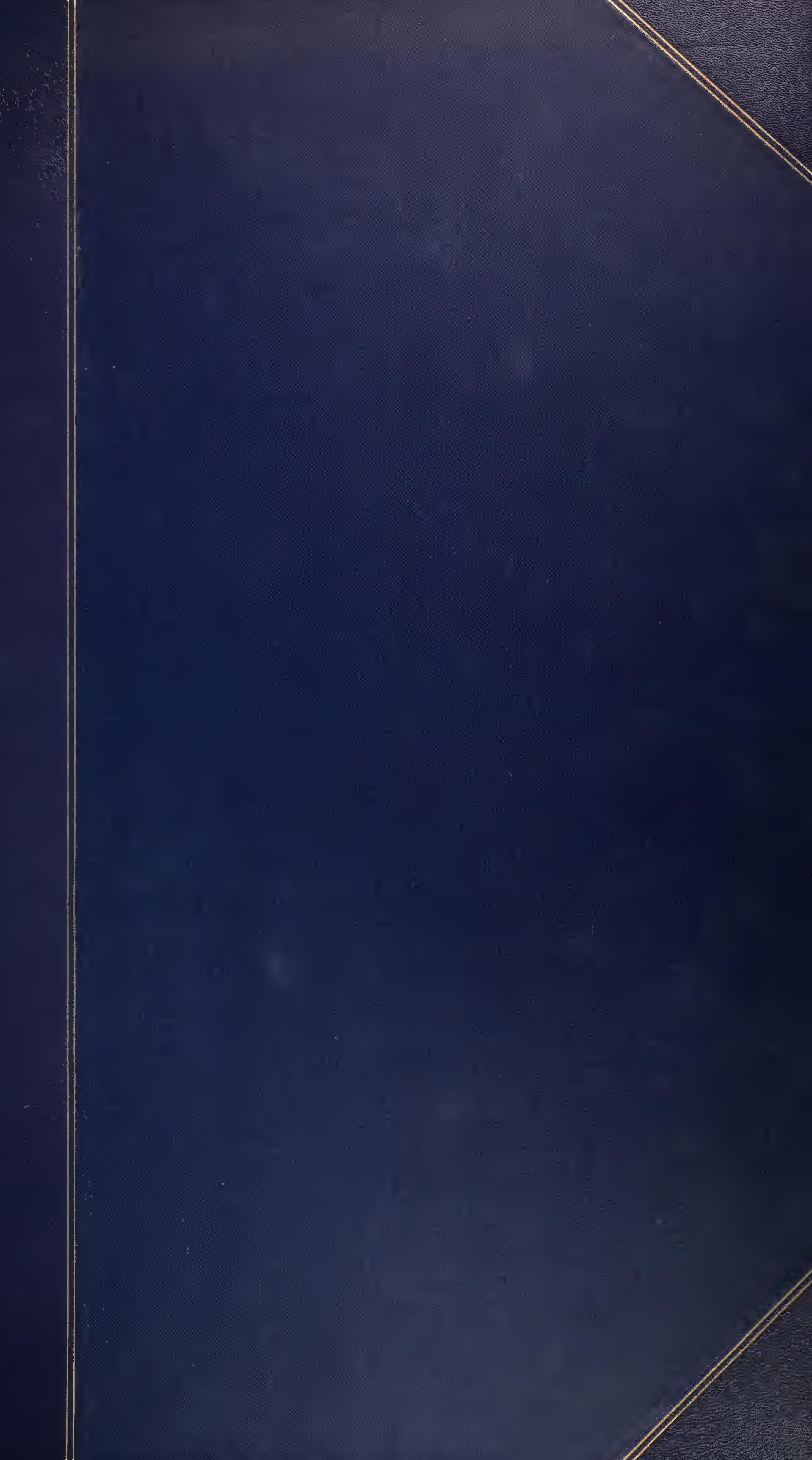








\title{
ORNITHORHYNCHI PARADOXI
}

\author{
DESCRIPTIO ANATOMICA
}

A U C 'T O R E

IOANNE FRIDERICO MECKELIO.

ACCEDUNT TABULAE AENEAE VIII.

$$
\text { L I P S I A E }
$$

A P U D G E R II A R D U II F L E I S C H E R U II .) 
SOCIETATI REGIAE LONDINENSI

I). I) (1)

$\begin{array}{llllll}\boldsymbol{A} & \mathbf{U} & \mathrm{C} & \mathrm{T} & \mathrm{O} & \mathrm{R} .\end{array}$ 



\section{E O R N I T H O R H Y N C H O.}

\section{$\begin{array}{lllllllllllllll}\text { I } & \mathbf{N} & \mathbf{T} & \mathbf{R} & \mathbf{O} & \mathbf{D} & \boldsymbol{U} & \mathbf{C} & \mathbf{T} & \mathbf{I} & \mathbf{O} .\end{array}$}

§. 1.

Ornihorhynchum et Eelidnen sen Tachyglossum ad animalia maxime memorabilia, praceipue altiorum classinm, pertinere, rel exinde clucet, quod Ornithorhynchum Zoologornm principes pro animali fictititio atcpe conposito habuerint. Hinc equidem ea semper in deliciis habui el jurenis, quatenus licnit, studii mei descriptione osscae cornm compagis testimonimm dedi ${ }^{1}$ ) gratissimo autem dono aestate anni MUDCCXXII. ab anicissimo Green, Chirurgo Londinensi, Ornithorhynchum marem, integerrimum, spiritu rini optime scrvatum accepi. Descriplionem completam alcue exactam miri animalis desiderari diu dolens, co lubentins looe opus suscepi, quod, hoe pracfixo scopo, melins in unirersum perscrutari atque scieutiae utilins solemus. Hieme anni MbCCCXXII. ad XXIII medio anatomian fere totam absolreram, quun perin, quatenus potnerim, perfectam publici juris facere cnpicus, anicis Londinensibus, ut feminam quoque milterent, literas dedi. Aestate anni Mn:CCCXXII. jam ad finem rergente, el. 1lomins ope el. Grecnii rolis satisfecit. Specimen loc, quamvis mimus et aperto abdomine ipsinsque risceribus exemtis mutilum, tanen tum ut supplerem atque emendarem, quac in primo observavi, tum ut memorabilissima nommulla inrenirem, nonnisi sexni feminino propria, simulque ad animalis natnram pertinentia, utilissimum milhi fuit. Thune statim descriptionem mean edidissem, nisi, minns bene ralens, et lnuic et aliis operibus vale diece coactus fuissem. Cum reliquis tamen et loc opusculum citius prelum reliquisset, nisi acstate practerlapsa, tum ut salutem coufirmarem, tum ut nomnulla ad anatomiam comparatam quac spectant instituerem experimenta, terlia vice in beatam me contulissem Italiam. Jan snperalis obstaculis omnibus, opusculum orbi literario trado. Diligentiam, ni fallor, haud quis negarerit; perfectins finturum fuisse opus, si major specimimun numerus, si recens occisorum praesto fuissel, lubeus concedo, atlimen rel sic me neque inutile suscepisse, neque noris inrenlis orbmn paginas sequentes probaturas esse spero.

\section{§. 2.}

Antequam opus ipsum aggrediar, breris notitia ordine chronologico componenda corum, quae hucusque doeti de Ornithorhyncho seripsere, hand inntilis videtur.

Descriptiones el fignrae primae totins aumalis vel nomunllarnm ipsins partium in Anglia el Germania, ni fallor, inde ab amo MbCCXCFIII. ab Shavvio ${ }^{2}$ ), el. Homio ${ }^{3}$ el Blumenbachio ${ }^{4}$ ) fuerunt exhibitac.

Mox amo IInCCCII. cl. Itomilus ${ }^{5}$ ) haud rituperindam miri animalis descriptionem anatomicam dedit, praceipne ad riscera spectantem.

1) Ostcologic der Echidna hystrix und des Ornithorhynehus paradoxns. In Beitr. zur vergl. Anatomie. Bd. 1. II. J. S. 64. Taf. 9 .

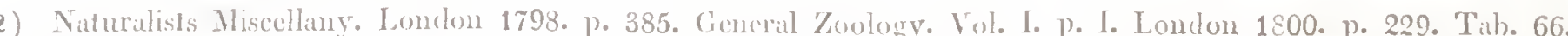

3) Some observations on the structure of the head of the Ornithorhynchus paratoxus. Philos. Transact. 1800. p. 432. T. 18.

4) a. Licher das Sclmabelthier (Ornithorhyuchus paractexus) ein nenentdeektes Geschlecht von Säugethicren aus dem fünften Welttheile. In J. A. Vo iggt s Magizin fur den neuesten Zustand der Naturkunde u. s. W. Bd. 2. 1800. S. 205 etc. — b. Abbildun-

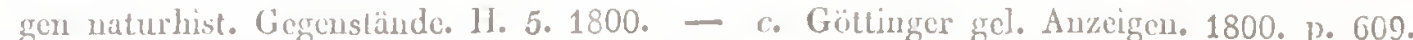

5) A Deseription of the anatomy of the Ornithorhyuchus paradoxus. Philosoph. 'I'ransact. 1802. p. 67. - Description of the Ornithorhynchus Hystrix. ibid. praecipue p. 356. 357. ubi nomulla prioribus addidit ct cmendavit. 


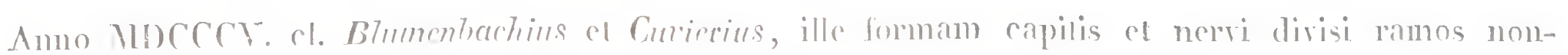

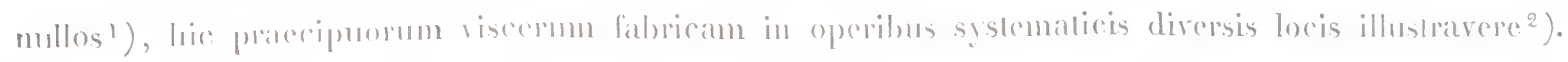
adicler $\left.0^{3}\right)$.

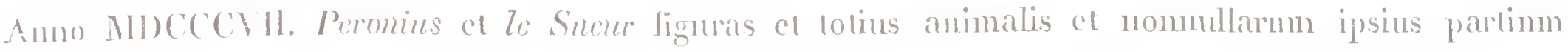

Sequente ammo a me descriphio sceleli Oruilhorhynoli et Folhidnes exhibita fuil ${ }^{4}$ ).

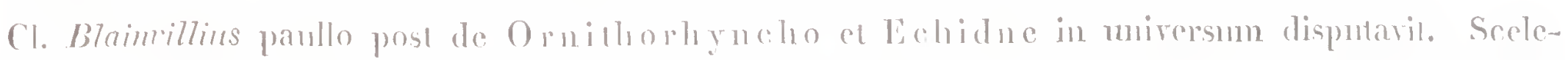

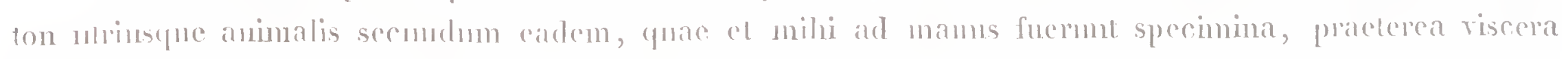
nommulla descripsil, el ex ipsius, reliquorumpuc dissechionibus ea ad mammalium classem pertinere (ronchisil ${ }^{5}$ ).

Cl. Lecelims paucos post ammos fisuram externam dedit $\left.{ }^{6}\right)$.

Mox cl. Genffroyus de ossihus trmeri, praceipue sterni el extremilatum anteriorum sermonem $\left.\operatorname{locil}^{7}\right)$.

Dein Blaimillits de ralcaris masculi struclura cogit $\left.{ }^{8}\right)$.

Eodem tempore el. Ilomius nommulla de modo gencrationis horum animalinu protulit ${ }^{9}$ ).

Mox cl. Risdolphius denuo ralcar illustravit ${ }^{10}$ ).

Dein Traillius et Mitlizs oriparam Ornithorhynchi naturam et calcaris perforationcm confirmare sluduere ${ }^{11}$ ).

Ammo MnCCCXXIIr. equidem, de Oruithorlignoho srripturns, primo summam corum, quac

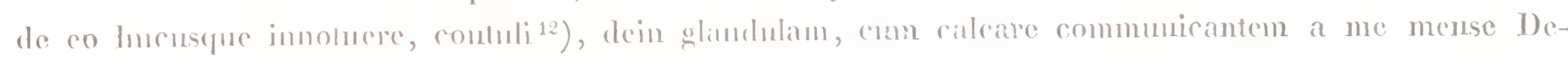

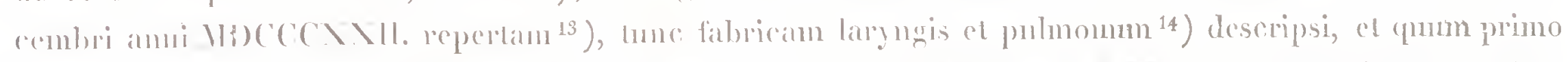
forcam in pede poslico feminino, doin mamman molimis ami libCCCXXII. diebus detexissem, utrius(jue descriplionem cum (l. Froriep, nlt eam publici juris fareret, communicari ${ }^{15}$ ).

Onamor fore menses, postynam a me glandula femoralis descripta fuerat, et cl. Rudolphius ejus-

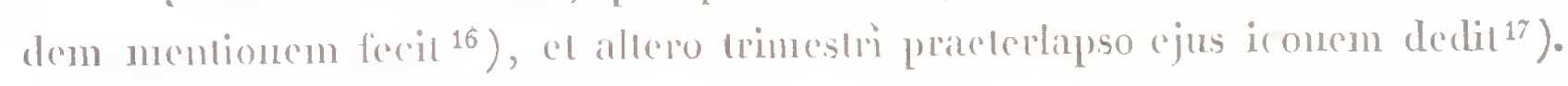

Dein cl. Knorii de Ornilhorhyncho mann scriptum in ol. Froriepii diariis breviter exceptum legimns ${ }^{18}$ ), de quo, antequam prelum reliquerat, jam dic 25 . mensis Julii luujus anni cl. Jameson milii literas dederat.

Novissimnm, quod nulit saltem innotuit, opusculım, cl. IIoerenii ${ }^{19}$ ) est, quo 1) praccipue differen-

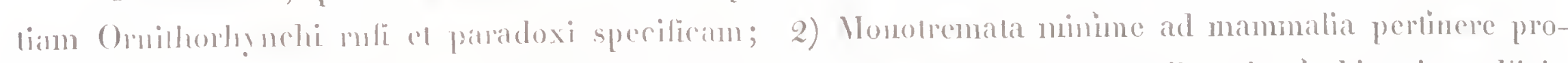

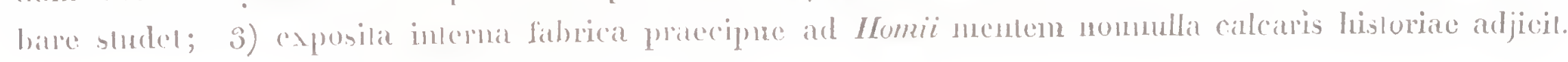

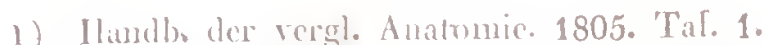

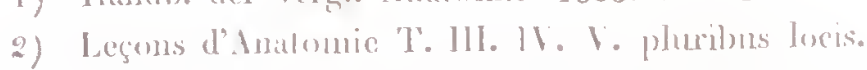

3) Voyage de diconverles anx terres utlstrates. 180\%. 'Tal). 34 .

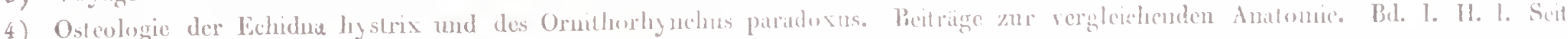
64. 'T'alb. 9.

5) Dissertation sur la place que la famille des Ornithorhynques at des Lechnidés dont ocenper daus.s les séries nalurelles. Paris 1812. 4.

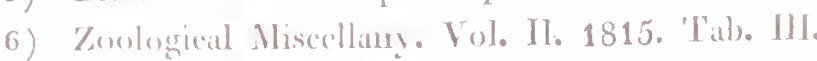

7) Philos, allat, 'T. I. Paris 1818. Tial). Il. Tiggo 19.

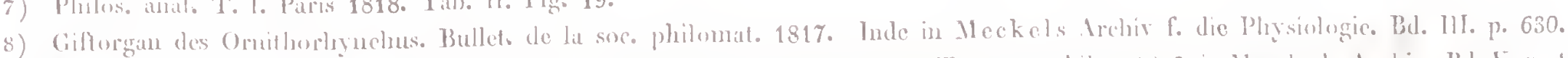

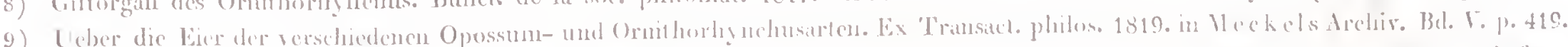

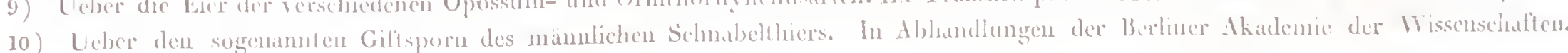
1820. 21. 1). $232-236$.

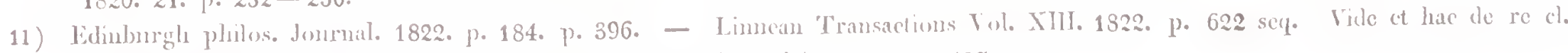

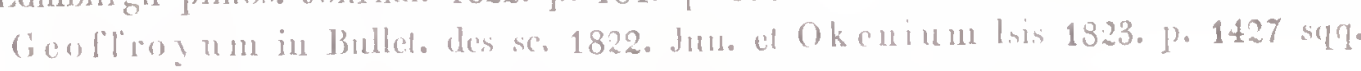

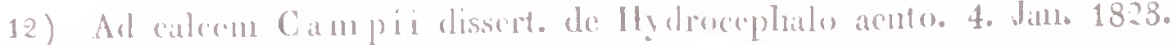

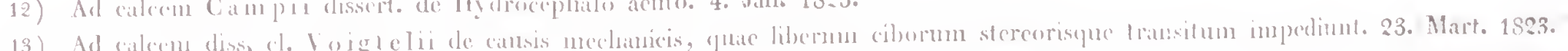

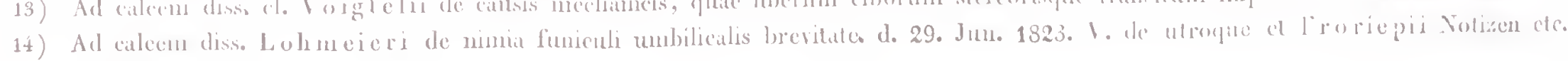
1824. p. 106.

15) Freit idem el. vir in ipsius Vulizen cle. Jan. 1824. p. 144.

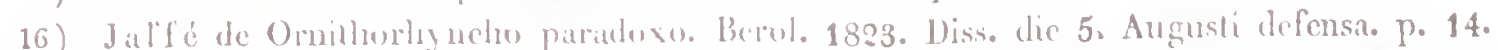

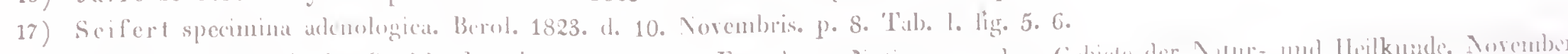

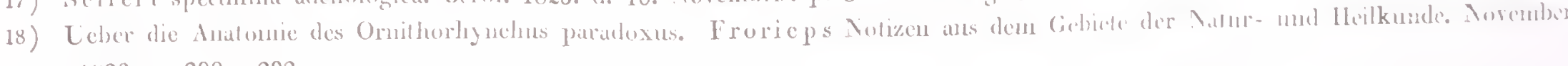
1<23. p. $290-292$.

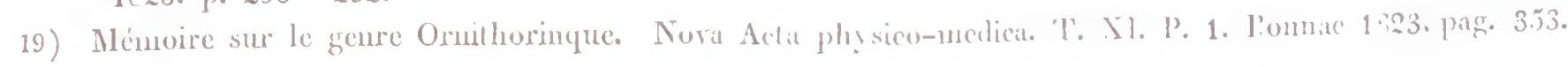


IIoc quamris jam ante scripta, et mammarum et glandulae femoralis pracsentian probantia edilum sit, Jaec saltem claro riro hand nola fuisse videantur, tancen ullimo loco addnxi, tum quia nomnisi ammo MIDCCCXXIII. appannit, tum quia mili nomnisi his ipsis diebus, jan prelo opusculum tradituro, innotuit.

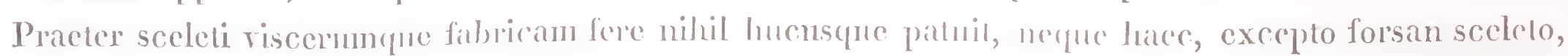
facillime scilicet parando, perfecte innohere, hine hand imutile duxi, animal diverso respectu mirum

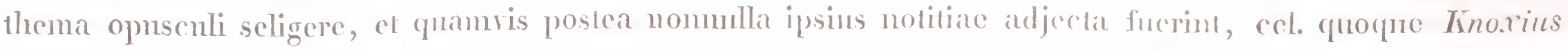
post IIomium proprium de codem publicareril in Actis societatis Wernerianis opusculum, tamen idco non destiti, quod, qui umum vel duo tautum specimina perscrutandi nactus est occasionem, necessario quacdam practervidet. Abunde hoc probatur listoria glandulae fenoralis in mare, magis etian mammae in femina, quae ab iis, qui vel abdomen apernere, rel sceleta confecere, quomodo practervideri pounerint, vix conceperim, nisi, multos anatomicos nimis aliormm oculis manilusque uti, nimis notum esset. Accedit (quod cl. Lino.rius feminam haud dissecuisse disertis rerbis dicilur ${ }^{1}$ ).

1) L. c. pag. 292 . 


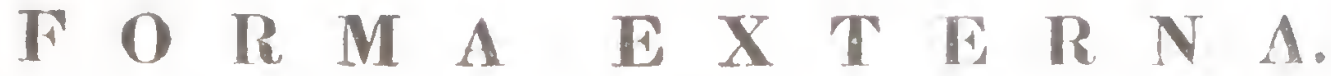

§. 3.

Miram animalis nostri formam externam, quamris jam a magni nominis viris, quos jam in antecedentibus adduxi, plus minusve accurate fuerit descripta, exactius adumbrare hand supervacunm duco. Hunc in fincm prinno descriptionem, dein, ut accuratius omnial pateant, dimensiones quoque addam.

Corpus totins animalis planum, a facie superiore ad iuferiorem compressum est. Caput et canda ejusdem fere longiludinis et dittitudinis, reliquo corpore longe planiora observantur, ratione longitudinis latissima, pedes respectu reliqui corporis breses.

Caput et quoad hahilum extermm fere luculculius quam in ullo alio animali in partem posteriorem, cranium, et anteriorem, faciem, dividitur, (pmun illud, ad modum totius corporis pilis, hace substantia coriacea, duriuscula, pilis omnino destituta, obtegatur.

Pars facialis posteriore vix angustior est, sed longe planior et lumnilior, antrorsum sensim paullulum latior et depressior, margine leniler convexo antice terminalur.

Substantia coriacea, qua tegitur, flexilis, ad margines anteriores et laterales lincarum 1, 5, in posteriore nomisi dimidiac crassiludine gaudet. Per tolam îsius extcusionem maximo mumero parrulorım foraminum, proxime sibi adstantium, instruilur. Dividitur in dimidium superius el inferins, per maximam longitudinis parten rich oris separata. Pone richum huns per guatuor linearum spatium ab utroque latere confluut, ita tamen, ut sulco, retrorsum et extrorsun versus marginem posteriorem ab angulo oris tendente, quodammodo separentur el margo posterior pone hujus sulci fincm posteriorem incisura triangulari, satis profunda et lata, notetur.

Adspectus maxime insolitus expansionc hujus massae extremi posterioris supra partem cranii anteriorem oritur. Revera quadrans ipsins circiler postremus partibus subjacentibus non unitur, sed omnino libere retrorsum ita protenditur, ut cutem subjectam pilosam tegat. Pars haec reliquna massa tenuior et mollior, sinnul et in facie inferiori lncidioris coloris est. Qunm enim relicqua, praesertim in dimidio superiore, fere nigra sit, facies hujns partis inferior, ilidem libcra, in dimidio postcriore, reliquae cuti pilosac, in quan retroflexa abil, proxima, flari coloris est, dimidiun anterius obscmins quidem, brunei coloris, sed facie opposita longe clanius. Sic zonae in lac ficie inferiori formantur omumo colore distinctae, nec sensim in sese transentes. Practerea facies hace interna ab externa et majori mollitic et totali punchulorum, quac jam indicavimus, defectu distinguilur.

Habilus hic valde mirus revera adspectum pracbet impositae reliquo capiti larrae, et, primis post repertum hoc animal temporibus nonnullis naturae scrutatoribus suspicionem movere potuit, animal, saltem quoad lianc ipsins structurae partem, arte mutalum esse.

Dimidium hujus massae superius inferiore crassius, durius et quinta circiter parte longins est, tota hac sectione anteriore ante inferiorem protensa. Pars postrema, libcra margine posteriore, Ieniter convexo, versus extrema lateralia paulhnlum inciso, circumscribilur.

In ipsius facie superiore, al initium quadrantis anterioris, nares poumulur, lincae medianac proximae, ut rix lineac spatio separentur, salis amplac, ellipticae, oblongae, retrorsum et extrorsum paullulum divergentes. Silum ad totins hujus superficici directionem tenent ommino horizontalem, nec nisi foramiua referunt, nulla elevalione vel ullo alio modo a reliqua superficie distincta. Deorsum ad foreas lineam circiter profundas ducunt, quae retrorsmun protendumtur.

Massae, quam jam describimus, dinnidium inferius superiore non tantum modo, quem diximus, brevius, sed magis etian, parte scilicet tertia angustius est, ut igilur, excepto fine postremo, ubique ab illo tegahtr. Celerum forma fere eadem est. Differt tamen 1) ideo quod versus latcra quam maxime, et suljito quidem, allenuetur; 2) margo posterior in medio incisura notetur; 3) pars posterior libera non 
ad rationem tolius longitudinis, sed etian absolnte, major sit; praeterea coloris paullo clarioris est. 4) facies ipsius superior, oris caro obversa, in quadrantibus tribus posterioribus per dimidium ipsius externum emineutiis transversis, maxime approximalis circiter viginti pracdita sit, quarmm in dimidio supramaxillari ne vestigium quidem perspicere polui, quamris hace struchma et luic adscribi videatur.

Sic v. g. cl. Cuvierius os Oruithorynchorum cum anatino eo magis couvenire monet, quod margines ipsius eodem modo lamiuulis transversis gaudeant. ')

Ceterun laninae hae duriusculac, retrorsum angnstiores, eodemqne modo longiores, magis versus lineam medianam protenduntur atque mitus ab se invicem distant.

Rictus oris inter massac dimidium superius et inferins positus, parabolicus, exacte marginibus externis et lateralibus dimidii inferioris definitur, apparet igilur minime, ubi animal a dorso conspicitnr, ubique a circunferentia massac superioris certe quatuor lineas distans.

Cranium globosum est, collo haud latius, imo arctius, ab extremo anteriori versus posterius latescens.

In facic ipsius superiore oculus et auris externa poumtur, propter parvitatem et elevationem, quam exhibent, nullam et summam pilorum, quibus circumdantur, spissitudinem, aegre invenienda.

Oculi medimm fere spatium inter personae facialis marginem posteriorem atpue aperturam meatus andilorii externi tenent, ab lıoc dnabus lincis, ab illo duabus cum dimidia, a facie capitis laterali septem, a linea mediana quinque lineis distantes.

Apertura palpebrarum rotunduscula, vix muius lineae diametrum metitur.

Meatus anditorii exterui ostium exterumm lincac circiter spatio magis extrorsum ponitur ocnlo. Foramen refert rotmolusculnu mins cum dimidia lineac diametri, ad meatum conducens, pollicis longitudine deorsuu, iutrorsum atque retrorsum desceudenten.

Collum breve, seusim versus thoracem dilatatur. Thorax et abdomen eadem fere latitudine gaudent, abdomine tamen paullulum latiore.

Cauda plana, latissima, sursum convexa, deorsum concava, abdomine hand dimidio angustior.

Extremitates nonnisi quoad pedes prominent, breviores igitur videntur. Anteriores iu extremo libero posterioribus plus dimidio latiores sunt, et longe magis illis extendi possunt, propter amplitudinem et laxitatem membranarum interdigitalinm longe majorem.

In anterioribus membrana natatoria a basi inde nnguinum secedit, ut omnino liberi, nec facie inferiore tecti promincant, sed plus quam lougitudine digitormm ipsormu ante corum apices protenditur. Itidem ad marginem maums extermu et interdum duarum ac trimm linearum decurit, versus digitorum basin scusim eranescens. Hinc maxina manus amplitudlo pendet. Meubrana natatoria practerea in tractibus, digitis respoudentibns inde a phalangis mugicularis basi usque ad marginem libernun versus facien superioren scnsim valde inflatur, incrassatur, ut lincac fere longibudine ante reliqumm marginem promineat. Sic margo hic leviter nudulatus apparet. Praeterea denuo satis luculente ex radiis lis inflatis, digitis respondentibus, secundus, terlius et quartus in medio incidıntur.

Ungues lougiusculi, fere plani, crissi, obtusi. Longissinums nedins, panllo minor quartus, minor etiam sccundhs, sequente primo, minino quinto. Jiscrimina non nisi parra.

Extremitas posterior anteriore vix brevior. Talipedem refert, facie dursali extrorsum, plantari introrsum versa.

Membrana interdigitalis longe maxima ex parte ad unguium radicem terminatur, excepto tamen halluce. Hic enim infra mugnem usque ad ipsins apicem producitur, libero, ut in extremitate anteriore, undequaque ungue.

Qumm non ante hallncem, nt in extremilate anteriori, protendatme, haec forma nonnisi vestiginum illius est, quac omni respectu longe magis evoluta in anteriore occurrit. Cetermm et incrassatio, cujus in hoc artu mentionem fecimus, in hac expansione pedis posterioris optime perspiciur.

Cuviertus, nomnisi membranan interdigitalem anteriorem ante migues protendi, adnotaverat ${ }^{2}$ ).

Memorabile est, qunm ungues quatuor externi arcuati, a lateribus compressi, aculi sint, unice internum, membrana lac protensa pracditum, forma auteriorum gaudere. Ex his secundus et medius acquales, tertius paullulum minor, minor ctiam hallucis, puintus minimus.

1) Règne animial. 'T. I. p. 227.

2) Ibid. I. 22\%. 
Culis totius corporis, excepta cauda, tegitur pilis rectis, mollissimis, tenuissimis et spississimis, ut iu linea quadrata ad ter centum numeraverimus. In dorso cum Ilomio ${ }^{1}$ ) nonuhil breviores quam in abdomine inveni, ut isthic sex, hic novem circiter lineas aequarent. Simul dorsales obscuriores abdominalibus, nou anten in femina lit quam in mare lucidiores ${ }^{2}$ ).

In caulà et extremitatibus subito longe crassiores sunt, durissimi, in his eliam multo breviores, ut vix duas lincas habeaut. In extremitate anteriore dorsum digitorum et tola palma omuino nuda sunt, iu posteriore pili ad medium usque unguhum dauntur unudà plantâ. Altameu in margiue ulıari manns et peroneali pedis pili usque ad extremum iuferins adsunt, praecipue in pede longi, qunn ad quatuor lineas accedant, et fere ad unguis ipsius extremum protrahantur, extrorsum directi, spissisśsimi praecipue pedis latitudinem valdopere augeant.

Candae facies inferior in ntroque individuo pilis instructa est longe berioribus et rarioribus. In mare line fere uuda est, in femima nomisi plaga ovalis adest, pollicem vix longa, dimidium lata, pilis omniuo destituta, quod ntrum a sexu, au actate, an casn pendeat, in medio relincpuam. Cl. Homius calvilicm hanc apparentem adnotans, de discrinume, quod diximus, nilit monet.

1) plicem pilım, ab IIomio memorilum, praeter discrimina modo dicta, exactissine omne totum corpus perquirens, nullo modo invenire potui, ut hauc fabriciun certe nonuisi Oruilhorhyncho rufo conveuire putem. Neque cl. Iloevenius hanc fahtrican invenisse vidctur ${ }^{3}$ ). Quanvis igihur cl. Peronius et diffcrentian pilorum specilican, quam iuvenisse sibi visus est, figuris expresserit, equidem adluuc de fabrica ipsa vehementer dubito, specie forsan nomisi conghtunatione pilorum nata.

א. 4.

Ad complendam descriptionem exteruam inensurne distantiarum praecipuarum perlinent neque descriptionibus, neque figuris, quanvis accuratis, omnino sistendae, et has igitur sequentibus ita damus, ut pedem Parisinum adoptenus.

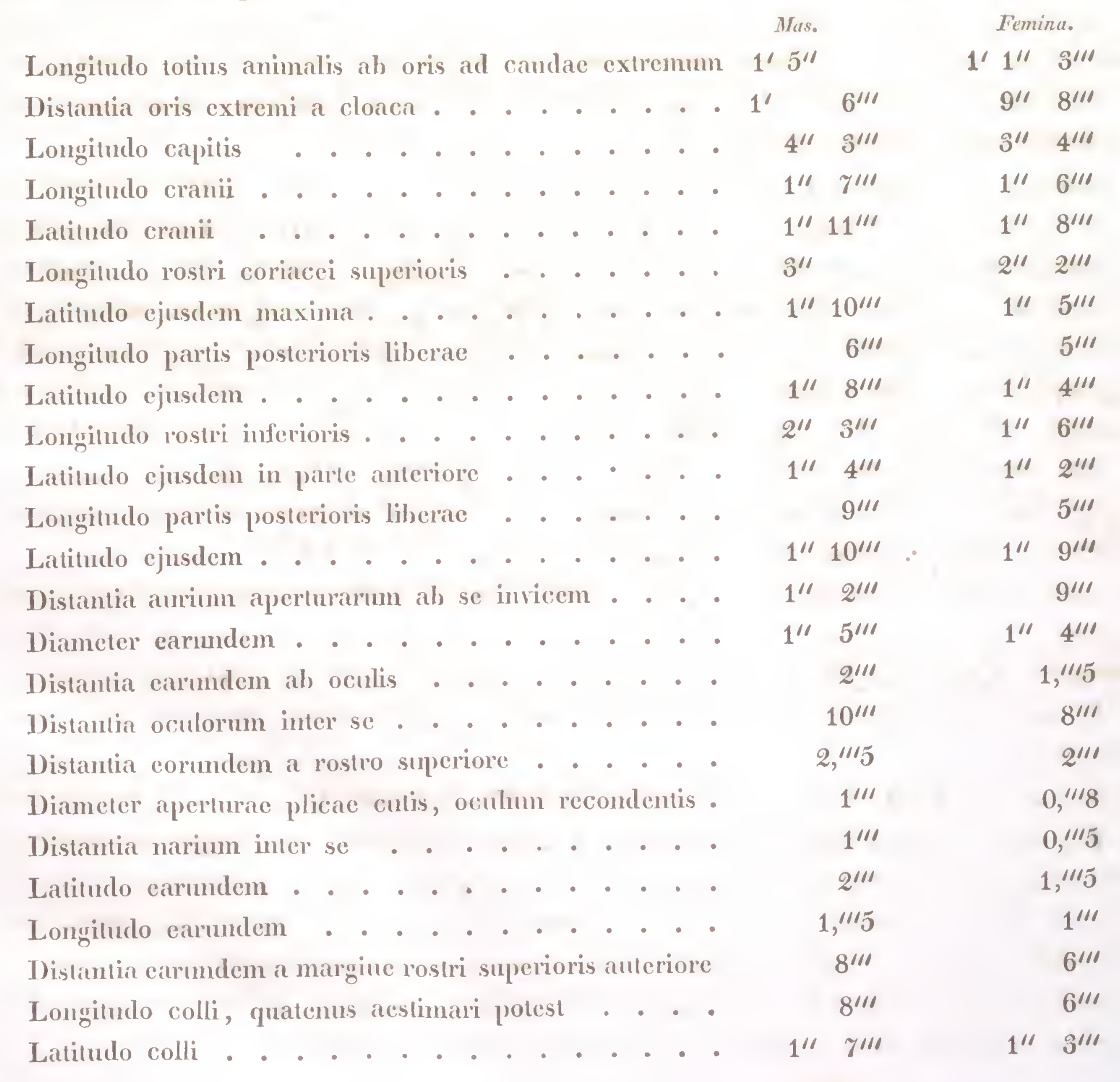

1) 11 o me Phil. Tr. 1802

2) $110 \mathrm{me} \mathrm{l.}$

s) I.. c. p. 372 


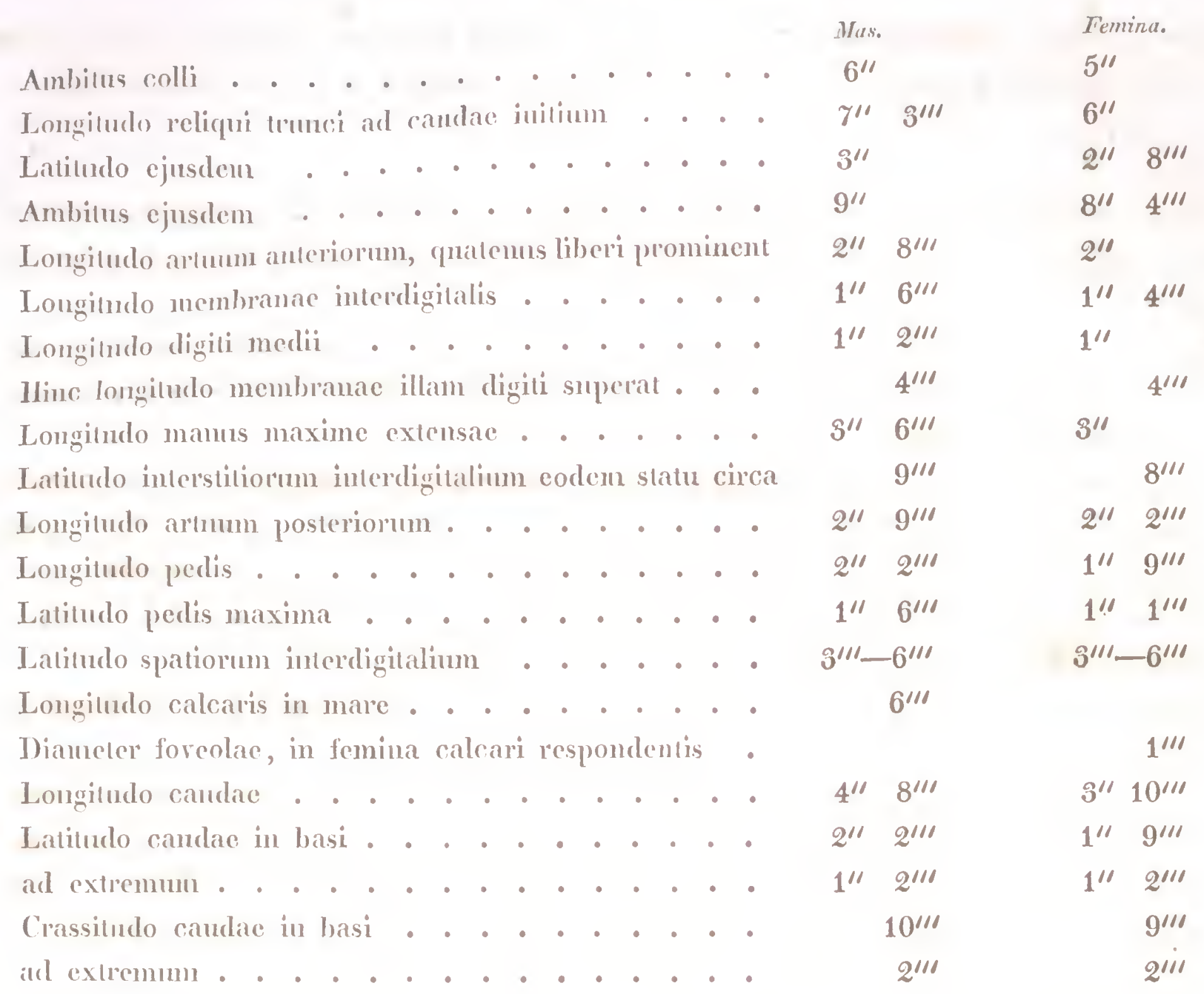

\section{\}. 4.}

Pondus Ornithorhynchi maris cxacte libras civiles duas, vel uncias triginta duas, fomiuae unam cun dimidia aequabat, ut, si circiter dnas uncias visceribus abdomimalibus, mixima ex parte deficientibus, tribueris, pondus feminae libram unan cum decem unciis explevisset. Discrimen pro magnitudine minus, sine dubio ob summam manmarum in femina crolutionem.

\section{§. 5.}

Extremilate posteriore differentiam sexualem, valde miram, offerri, vel minus perilos haud fugere puto. In mare, hane ipsam ob rationem at) omutibus salten quadrmpedibns distincto, calcar, vel aculeus, satis miaguus, apparet, primo pro digito sexto habitus, cujns cum pirtibus internis aretissime cohaerentis, earunque nonnisi extremunn extermmm sistentis descriptione fusiori hic supersedeo, accurate cunn gencrationis organis de eo acturus.

Loco acnlei in mare inveniundi, si anctoribus, qui adlunc de Ornithorluyncho scripsere, fidem habemus, in femina omniuo uiluil observitur; rem autem in specimine meo accuratius perscrntatus, utique fabricam hic peculiarem observari, cujns habitmm saltem extermum hic deseribendum puto.

Foramen scilicet invenitur, in ipso plantac initio, uhi pili desinunt, fere in ipsins medio, pantlnhum tamen vicinius a mingine peroneali distans, lineac dianetrum vix aequans, lineas circiter duas profindum, vallo parum elevalo circumdatum. Culis, iu planta fusca, circa idem el in ipso laetiore albido colore gandet. Cavmm pilis destituitur. Fablicam internan infra fusins dicam.

Hace pars num nsum alifuem habeat, an rudimentum tantum inutile calcaris sistat, cujus locum tenet, ommino nescio. Neque structura glandulosa, neque meatu exripitur, sed mera cutis impressio videtur. Vasa et nervi hand insolita copia hanc partem adenut. Attamen sensus exquisitioris sedes esse potest, forsan inmisso calcaris succo sollicitati. Relationem inter calcar et hanc foveam adesse, jam locus aequalis suadet. Sic igitur glandula femoralis marium, in vuluus venenum fortius instillans, aphrodisiacun forsan generat, cui sententiac aretnn, quod inter secretiones complures et affectus in universum, aphrodisiacos in specie obscrvatur conumbiun, haud parmu favere videtur.

\section{§. 6.}

Practera differentiam scxualem nullam, salten certam, invenire potui. Pilum in ablomine minus obscurum huc nihil facere, jam monui, et de candac discrimine dubio egi. Num magnitudo con- 
stans pracheat, nescio. Ilomius certe marem quoque femina majorem invenit, quamris ratio minor

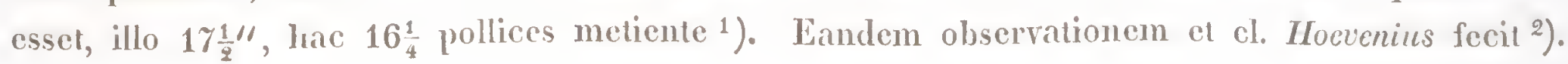

\section{ค. 7.}

Peracta externa animalis nostri descriptione priusquam ad stucturam internam transeamus, haud inutile fuerit, adjecisse criticam brevem ligurarum habitus externi, hucusque publici juris quae fuerunt fartac.

Omues hoc vel illo modo plus minusve graviter peccant, praccipue quidem, quia fere omnino ad exemplaria siccata adumbratae fuerunt. Alter enim fous nimia omnium mensurae reductio esse potuit, quamris facilins evitandus.

Shavii vario modo errat. In universum ommia minus bene distincta sunt, forma nullius partis satis accurate indicata. Si, a rostri fine autico iucipientes, corpus totum perhustrando ad caudam externam progredimur, primo nares invenimus forsan nimis parras, certo falsissine rotundas, quum luculenter ellipticae sint, dein longe nimis inter se distantes, yuum in figura, ad tertiam normalis magnitudiuis partem reducta idem ac in adulto, quen coram liabeo, intercedat spatium.

Rostrum superius tum contractione nimia in parte media, tum minime exacta expressione marginis posterioris peccat.

Rostrum inferius munquam ita, ut liac exprimitur figura, a superiore remoreri potest, quum, quavis dati opera, vix ita id deprinere possim, ut duarum utrumque linearmm spatinm intercedat. Quamvis practer elevationem et depressionem motns quoqne detur lateralis, hic quoque minimus est. In figura Shaviana autem rostrum iuferius tota ipsius latitudiue superins transgressum est, ut utercpue ipsius margo lateralis, quamris hatud remoto superiore, spectetur.

Peccant praeterea dentationes nimia altitudine, contra munero justo minore. Et spatimun nimis breve occupant, quum revera magis antrorsun protendantur.

In capite, saltem si mea exemplaria contulcrim, circulus albus, oculum cingens, latior naturali est, oculus longe nimis a rostri superioris margine posteriori distat.

Eodem modo et punctum nigrum, auris, ni fallor, indicium, nimis ab oculo distat.

Collum nimis longum et angustum est, ut extremitates anteriores nimis magno spatio interjecto caput sequantur.

Reliquum corpus nimis rotundum, cauda justo nomuihil brevior est. Falso in extremo posteriore bifida apparet, sulco medio satis profundo, quum revera margine obuso, couvexo terminetur.

Mirandum quoque, magnam pilorum caudalium et reliqui corporis differentiam ne quidem fuisse indicatam.

Extremitates anteriores primo nimis longae sunt, dein nunquam talem situm maxime coactunn offere possunt, ut, modo hic indicato, facies manus exterua omnino sursum rertatur, autrorsumn tantum et extrorsum flectenda.

Praeterea, quae ungues esse in figura videntur, revera toti digiti snut, ungues ipsi justo lourge acutiores.

Minus etiam recte digiti omnes, practer quintum, ejusdem longitudinis picti sunt, quum et primns et secundus terlio quartoque minores sint.

Spatium extremum digitorum atque marginem liberum membranae natatoriae intercedens itidem justo lougius est

Forma membranae natatoriac et in universum minus bene fuit exhibita, nec ullum adest indicium elevationum illarum longitudiualium, satis notabilium, digitis respondentium.

Extremitatum posteriorum digiti justo longe unajores sunt. Pessime praccipue calcar retrorsum vertitur, eâdem omnino cum digitis directione, sic fignra cum Shaucii verbis: „On the hind feet are "six claws, longer and more inclined to the curved form than those on the fore feet etc." consentiente.

Ungues posteriores anterioribus quidem curviores et acutiores, nimime autem longiores, contra breviores sunt.

Nonnulla ex his cum, si benignior esse relis, magnac mensurarum reductioni tribuere possis, dolendum, vitia eadem et in tabula adjecta, capnt, pedesque in magnitudine naturali offerente occurrere.

1) Ph. Tr. 1802. p. 68.
2) L. c. p. 365 . 
Qnamvis enim pes uterque nomihil accuratins fucrit redditus, tamen pessime hic quoque calcar omniuo eanden rum digitis servat directionem. Onac de capite antea censuimus, hic quoque offenduntur.

Cl. Blumenbachii figura, quanvis exemplaris sicci nounisi dinidian sistat magnitudinem, tamen Shanciana et Peronianis longe melior est.

In Peronii tabula, quanvis ralde reducta, optine magnum discrimen inter caudae et reliqui corporis pilos, illos rudissimos, hosec scriceos, expressum est. Bona quoque cranii candaeque forma. Contra corpus nimis altum el rotundum videtur.

Practerea multa alia rel maxime offendunt. Hortum primum est ratio longe nimis magna inter rostrun et reliqunm corpus. Qunu cuim revera partes duas metiatur decimas tertias, in Peronii fignra nomisi decimam, imo in Ornithorlyucho fusco ne undeciman quidem aequat. Hoc mde peudeat, nescio, qunn ne maxima quidem exsiccatione talis fabrica oriri possit.

Dein caudam nonuihil quoque justo breviorem putaverim.

In capite practerea narium et forma et situs omuino codem modo, quo apud Sharium, peccant.

Gravissimmm vitium in ocnlis notandum. Sou tantum nimis retrorsmun ponuntur, sed decnplo saltem naturalibus majores snut.

Aurinm quoque aperturas indicari potuisse putarerim.

Extremitates anteriores nunquam, uti hic expressum est, omuino pedum anteriorum crustaceorum modo angulo antrorsum acuto ita antrorsnum flecti possmit, ut collo parallelac ponantur, et apices monginm omuino extremum aulicum spectet.

Forna circnitus menbranae natatoriac minime exacta, quum omnino integra sit. Neque rectins ungues et arulie et lougitudine naturan relinquunt.

In figura Oruithorhyehi ruli maris pes posterior calcar ostendit si nou retrorsnm, tamen transrerso positum, quod, quantunvis pedem hunc torseris, nuuquam obrenire potest. Ungues justo longiores, cum auterioribus aequales sint depicti.

Homius liguras non totins quidem animalis, sed rapitis pedumque dedit satis aptas et jam citatis raldopere anteponcmdas.

Satis bene indicavit oculorum et annium magnitudinem, in prima quoque fignra, capitis fariem superiorem sistente, situm utrinsque organi, quod in tertia nimis retrorsmun posnit. Forma nariun arcu. ratior est, distautia tamen et hic justo longe major. Rostrum superius in medio marginis antici acutum, inferins excavatmm pinxit, quod num a desiccatione quadam, quanvis aninal spiritu vini condiumn fuit, an a fabrica individua pendeat, nescio. Rostrum superius extremo autico sursum convexum est, in uneis exemplaribus subito concarum. Figura pedis postici maris dextri, quan in altero volumine exhibnit, optime totius organi fabrican in muirersmu, practerea processmun membranae natatoriate inter hallucem et digitum secundum, dein calcaris forman atque situm normalem sistit, ab omnibns inlecessoribus mutatum.

Leachii Ornithorhynchi fusci figura, nisi quod ligidlior, et quoad colorem satis bona est. Lncisnrae plantarum bene expressac. Ocnllus nimis magnus videtur.

Hoevenii Ornithorhyuchi finsci et ruli icones, ad exemplaria gossypio expleta factae, partes pilis tectos satis bene exhibent, nisi forsan discrimen inter caudae et reliqui corporis prilos haud satis expressum sit, minime auteu naturae conrenit cutis facialis pars postrena hic quoque recta, ad perpendiculum posita. Facies pedesque, haud secundun naturam, macilenti et exsiccati sunt, membranac interdigitalis pahmaris incismris mullis notatae, calcar jessime retrorsm rersmun. 


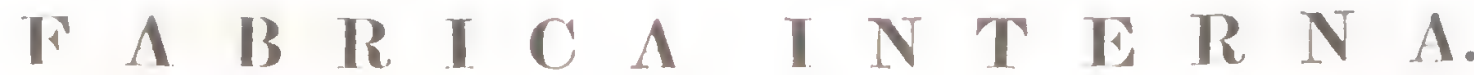

\author{
0 s s $A$ \\ 1. 8.
}

Scelcon Oruiluorly yuchi relicuis partibus melins illustratum esse, nemo mirahilur.

Praccipue in lac miri auinalis parte phurima occurrut, nexum cum mammalibus et ampluibiis clarissime iudicantia.

Columua spimalis quadragiuta novem componitur vertebris, e quibus septem regiouem cervicalem, septendecim dorsalem, duac lunbalem, tolidem os sacrumn, viginti el uma candiun couslitumut.

Numserum hutc et distrihutionem et ego ${ }^{1}$ ) et cl. Blaincillius ${ }^{2}$ ) (quum jan pridem dederimus, unper cl. Rudolphius, rim rertebrarum posteriorum ita mulandam censuil, ut ossi sacro quatuor, candae nomnisi noremdecim tribueret ${ }^{3}$ ). Huic sententiac pace cl. viri vix accedere possum. Absentiam rel praescutiam processumm spinosorum inferiorum in vertehrarum uaturam vix ullo modo influere puto, quun in aliis columnac spinalibus, r. g. cervicali, dorsali, lumbali et in mammalibus iuveniantur. Edemtala quidem, ob ossis sacri magnitudinem, pro cl. Rudolphii sententia stant, contra antem Amplibia militant, Ornithorhyncho proxima, nomnisi parro osse sacro et duabus vertebris composito gaudentia. Neque ani situm multum huc facere, auatomia comparata multis exemplis probat.

Vertebrac rariarum regiomm phuimum inter se differmut, ut in universum tantum satis magnac et forles dici queant.

Thoracicae et lumbales satis compressac sumt, et ejusdem fere magnitudinis, ut tamen ab anterioribus rersus posteriora sensim nomilnil crescant.

In basi arcus foraminc rotundo, satis magno instrumutur, nervis spinalibus et rasis exitum pracbente.

Processus spinosi parvi, in omnibus thoracicis, excepta ultima, retrorsum, in hac et humbalibus subito antrorsum inclinati. Obli(pni anteriores, praecipue in posterioribus, salis longi, transrersi nulli. Ouatuor anteriormm corpora in facic inferiore processu gaudent spinoso inferiore, conpresso, satis magno, cujus vestiginn, quamvis parvun, et in tribus sequentibus, denuo in quatuor thoracicis posterioribus, minus in utraque lumbali invenis. Ex processibus anterioribus primus relipuis longe magis compositus est, rassior, latior, in spinau median duasque laterales, longiores antrorsum excurrens, fabrica ideo praecipue memoramda, quod in avibus haud raro occurrit.

Vertebrac sacrales, aequales, minime concretae, subito dilatantur, valde auctis processibus transrersis, ossibus ilium unitis, haud latis, sed longis. Primae corporis facies inferior leniler excavatur, secuudae ad antecedentium typum eminentiolan longitudinalem medianam offert. Unaque processu spinoso, autecedentibus majore instituitur. Ex candilibus prima nomihil augnstior, secunda sacrales aequat. Utrique corpus magis cliam compiressum, processus spinosus latior. A prima ad seplimam dilatantur, ancta latiludine processumm trausversorum, momisi parro spatio disjunctormm, dein sensim rontrahuntur et extrorsum acuminantur, ut ex quadratis triquetri exsistant, nonnisi in ultima, triquelıa, onunino eranescentes. Vertebrae, exceptis ullimis sex rel septem, vix longitudine, sed maxime altitudine decrescunt, ut planissimac evadant, sensim et corporihns et processibus spinosis hac directione maxime diminutis. Processuss spinosus superior in decima ommino disparet, jam a secnnda diminulus. Ohliqui posteriores nonnisi ad sextan, anteriores ad deciman nonam usque adsmut, inde a decima sexta nomnisi tubereulum simplex, medimm referentes. A tertia ad decimam septimam processus spinosi inferiores, saltis magni, ad septimam crescentes, dein decrescentes, omnino cum corporibns concreti, aldsunt.

1) L. c. p. 82. 83.

2) I. c. p. $\%$.

3) Jaflé p. 12. 
Vertebac ecricales ex latissimis et altissimis sumt, reliquis tamen in universmm, exceptis caudalihus postremis, breviores. Omnes, practer Allutem, processibns spinosis superioribus el inferioribus instrunutur, quorum illi majores, ad septimam usque maxime decresennt, lii, minores, ratione onmino conIraria, longitudine, sed mulıo mimus, angentur. Processus adsunt transversi, alti, lati, radice superiore "t inferiore et foramine restchrali maximo, excepto Allante, instructi. Magnitudo a tertia sensim valdopere diminnitur.

Prima el secunda omnium maximae, latiludine inter se conveninut, Epistroplaco tamen magnitudine Allantem multum vincente.

Ille practerea a vertebris ommibus distinguitur 1) processn spinoso smperiore allissimo et longissimo, maxime conpresso; 2) fabrica dimidii corporis inferioris. Antrorsm scilicet convexum, parte smperiore dente salis magno sed temui terminatur, e cujus extremi antici lateribus ligancula fortissima ad partem circiter mediam faciei internac condylorm occipitalimm abeunt. Dens tohs non tautum, sed corporis quoque facies anterior tota cartilagine obducitur et, deficiente quovis processumm articularimm anteriorum restigio, clarissinc cormu confluxu in faciem uman, simplicem, nata apparet. Ceterum deus minime cum Allame, sed ante ipsum cum occipite modo dicto unitur, liganento tantum transverso, fortissimo, pouc ipsun decurente, in situ retentus.

Tertio numcro partinn a reliquis vertebris differt. Pum enim reliquae omnes, hand exceptis caudalibus, nonnisi unicum sistant os, hace quatuor componilur, corpore iu dimidimm anterins et posterius secedente, processus trausversi parte externa, simplici a radiec duplici, quacum foramen rertebrale format, scjincla.

Epistrophacum reliquas magnitudine rincere diximus, cni sententiae quonodo oppugnet cl. Blainrillius, tertian corvicalem ipso Epistroplnaco majorem pracdicans ${ }^{1}$ ), equidem ommino non intelligo, qunm dimidio minor sit, neque Allantem acquet.

In Allute, practer ea, quae jam diximns, 1) solito more, foramen pro medulla spinali maximum, in partem inferiorem et snperiorem, majorem divisum, adest. Hla denten Epistrophaci adnittit, minime antem cum co articulatm; 2) corporis facies posterior, ad Epistrophaci partem anteriorem, excepto dente, forea simplici circumdandam, excavatur; 3) processus trausrersi duplices adsmut, superiores, anteriores, lati et longi, inferiores, longe posteriores, minores, retrorsum et extrorsum directi, revera nomnisi radienm solemnimn disjunctione completa formati. Radices singulae inter se maxime distant, nullo modo convergentes. Jine ralde memorahile, et in Epistrophaeo radicem utrampue nounisi osse accessorio exteruo vera costa, uniri.

Processus articulares anteriores Inmiles, sed facies articulares longissimae, profundae, deorsum couvergentes, capsula unica, valde laxa, cum utroque condylo occipitis simul unimutur.

\section{§. 9.}

Costac satis fortes, rotundusculae, nomnisi modice incurvalae sunt, prima reliquis nomnihil latior et planior, ultima omninn minima. Ab ntraque magnitudine ita angentur, nt tamen practer priores et posteriores fere inter se arequales siut. Thberculum adest nullum, et nomisi capitulo rotundo crum corporibus rertebrarm jungnutur. 'Tredecim anteriores hinis, relipnae tres singnlis tantum vertebris respondent. Sex verae, relipnae spuriate. Modns mionis vermum com stemo non onnibus idem, prima enim rartiligine nomuihil angustiore ot breviore sterni manubrio muitur, quincue reliquae cartilaginibus quidem iisdem intereostilibus instrututur, sensin magnitudine anclis, non anten ad sternun usque extensis, sed extremo autico ossibus rectis, crassis, longis nectuntur, quornm cxtrema anteriora nexu mobili cum sterno jungmultur.

Sic igilur costa prima omnino ad mammalium typum cum sterno nuilur, in relipnis arimm et mammalium fabricae junctionem observas, qumm adsint ef costae illarm stemales, et cartilagines mammalium, illas et rertebrates intereclentes.

Costac spmriate solito more cartilaginibus iustruntur antrorsum et introrsun directis, omnibus, excepta nitima cum pemultima, in parte interna et antropiore maxime applinalis et attenuatis, ul, posterioribus anteriores tegentibus, scutum abdominile fiat. Practer yuatuor posteriores simul longissinae sumt.

Cartilagines omnes duriores smmt, et ussei aliquid luabere videntur, nomnisi antem a secumdat ad 
scxtam nsque rerae adsunt costae sternales, carlilagine rerî junclae. Cartilago prima manubrio synchoudrosi, costac sternates secundia ad scxlam capsulis sterno, spmriarum cartilagines musculis intercostalibus tantum laxîfue cellulosî inter se, jungnutur, minime antem, ut IIonius dicit '), substantia liganuculosa valde clastica.

(Guam dedi, descriptio haud ommino cum reliquis convenit. Sic Blaimvillius ${ }^{2}$ ) et Rudolphius ${ }^{3}$ ) costis reris o muibus cartilagines, interno extremo osscfactas tribumut, quod uescio an nuquan accidat. Equiden saltem nou in meo taulum sceleto, sed IFomiano quoque carlilagiuem primam nuspuam osscam invernio, (quamris in Echidne Parisiensi rerera et hace omnino osscfacta sit ${ }^{4}$ ).

Partem dilatatam carlilagimm costarum spuriarum Carus pro lamiua propria ossea habnit ${ }^{5}$ ), hand bene mentem Homii asseculus, cartilagines costarum spuriarum, in lanninas osscas plauas latasque abire dicentis ${ }^{6}$ ). Celernm discrimina lic adsunt, forsan ab actate pendentia, qunm in meis exemplaribus durities quidem adsit cartilaginibus costarnm, nimine antem rera natura ossea, Parisicuse codem modo se lrabeat, contra in Londincusi etian in spuriis, excepta nllima et peunltina, maxima cartilaginis pars osscaun exhibeat naturam. Qnamvis autem latec mulatio locum lrabeat, minime tanen laninae illate pro ossibns proproiis sunt habendac, aretis limitibus a reliqua cartilagine lrand distinctac. Contra coslac in secunda ad sextam sternales, reris vertebralibus respondentes, osseac omnino natmrae adsunt, a cartilaginibus costalibus discretae.

Hanc fabricam Ornitlorliyncho tantum inter mammalia adscribens, cl. Carus ${ }^{7}$ ) lıand $11 n o$ modo erravit, ynum non tantmm inter Monotremata et in Lethidnis idem inveuiri jam et cl. IIomius ${ }^{8}$ ), et $\operatorname{los}^{9}$ ) et cl. Blaincillius ${ }^{10}$ ) discrtis romis docnerimus, sed Myruecopluagis, Dasypodibus alque Celaceis quoque cartilagines costales in ossa mutatae adsint, quod de Delplino plio(a cena jam Tysonus ${ }^{11}$ ) et post emm Bonnalerrius ${ }^{12}$ ), de Bradypode didactylo Cuvierius ${ }^{13}$ ) adnolarelant.

Numerum costarum et ego iu prima sceleti descriplione, et post me cl. Blainvillius, quem tamen parum sibi conrenire miror, primo ${ }^{14}$ ) recte de septemdecim, dein falso de sedecim tantum loquentem, et Rudolphius ${ }^{15}$ ) ad septemdecim accedere diximus. Prorsus idem in ntroppe, quod coram est, invenio sceleto, ut igilur levem lic cl. Homius erroren commisisse videalur, nonnisi de sedecim loquens, artilice in dextro latere, ut videtur, nomisi quindecim, in sinistro seplendecim pingente.

\section{§. 10.}

De sterno sententiae auctormm differme, qunm partes, ab alijs ad hoc os rel hanc ossimm compagem relatae, ab aliis vario modo ad alias sceleti regioues referautur. Hinc, ut clarior res pateat, faciliuscue judicium ferri possil, anbignae partes omnes simul ita describendac videntur, ut primo tamen cerlas sistan. Hae costis reris interpommun et columnan sistınt maxima ex parte satis compressam, quatuor ossibus, synchondrosi nuitis compositam.

Hormm primum, maximum, sed teune, triangnlate, antrorsnm valde dilatatur, ut hic reliqnam colnmuam sexies saltem viucat. In utrayue facie spimulam longitudiualem gerit.

Primum hoc os e duobus dimidiis lateralibus componi, cl. Geoffroyus ${ }^{16}$ ) et Rudolphius ${ }^{17}$ ) nonnere, lıoc, si passum, sphalmate typographico defiguratum recte interpretor, addente, in adultiore specinnine

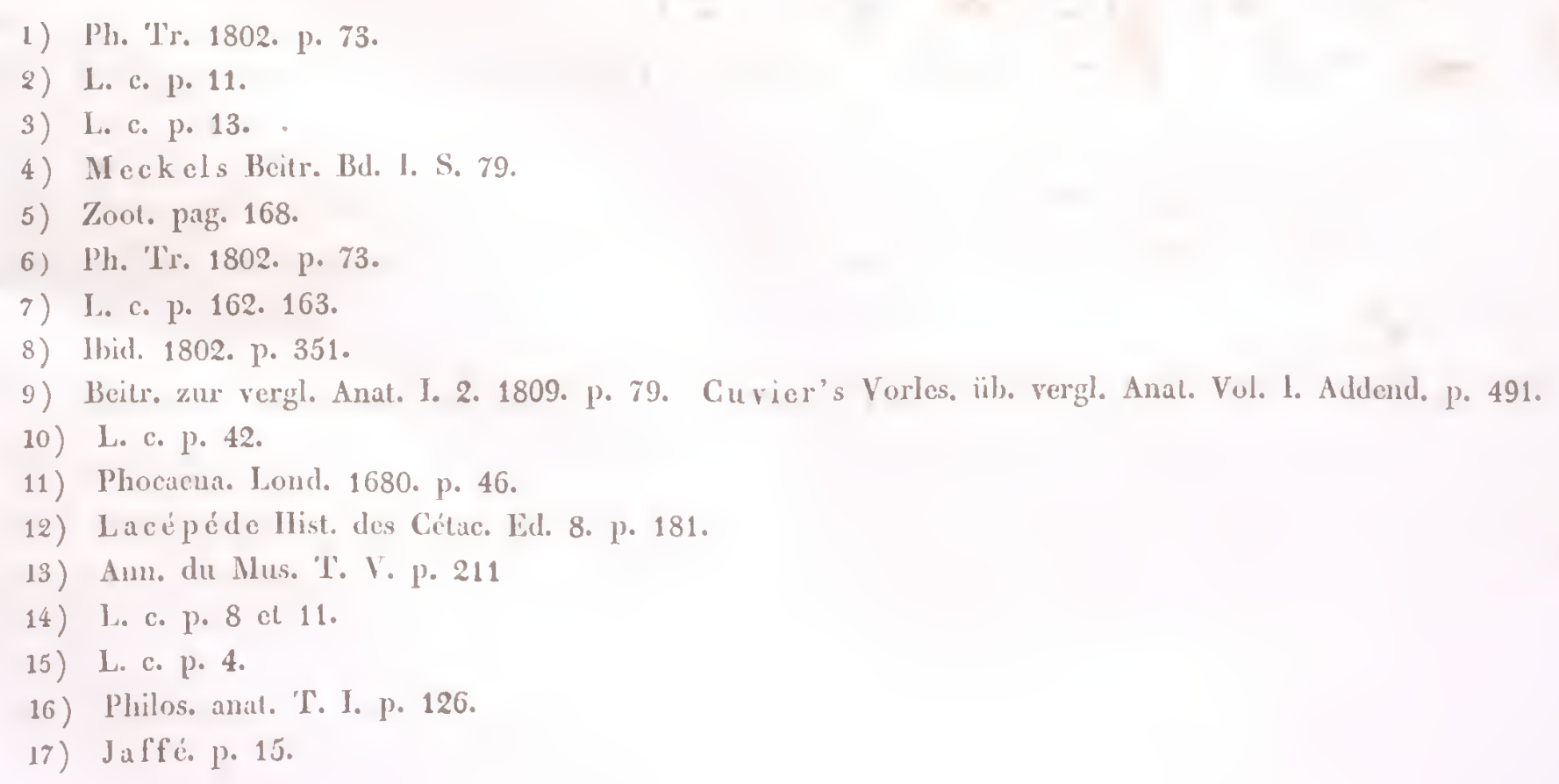


fissura disparente duo dimidia in umm os confluere. Equidem neque in scelelis, quac Parisiis et Londini vidi, neque in meis fissuram video, pated igilur, os hoc, noc, forsan semper, ex duobns nucleis fieri! Cl. Geoffroyus suo modo hanc separationem pro re inaudita et maximi momenti habet, ita exclamans: „De plus, cette même moitió est parlagée en denx portions par nue séparation longitudinale; seconde, mais bien plus esscnticlle différence. Car la large plaque est parloul un os impar, absolument partout, excepté les nonotrêmes, on sa division derient anssi m caractère étrange, exclusif, et bien propre ì marcher de frout arec tontes les autres de ees ílres éminemnent paradoxinx." 1 )

Dolendun, et hic rem nullius momenti pro maxima vendi! Utrumque dimidium concrescere jam cl. Rudolphii et nostra experieutia docuit. Dein falsissimum est, Inunc formationis modum nounisi Monotrematihus competere; nam in Monodonte monocerote, cl. Rudolphio in Berolinensi ${ }^{2}$ ), me in meo sceleto observantibus, res omnino codem sese habuit modo. Forsan idem toti Cetaceormm tribui convenit, qumm, et Tysono ${ }^{3}$ ) et me ipso sacpissime observantihus, foramen in manubrio adsit, quod in Plhocaenae sceleto semel fissma longitudinali ad marginem anticum usque extensum ridi.

Qıid, quod in homine interdum exaclissine ad enulem typum primam sterni vertebram formari, jam din et literis et fïgura docni ${ }^{4}$ ), cujns rei praeclarum exemplum postea vidi, observante idem cl. Ollone $\left.{ }^{5}\right)$.

Ex reliquis tribus, brevioribus, oblongis, longe angustiorihus medium nonnilil minus est. Primum, jam dictnn, cartilagini costae primac et secundac interponilur, secundum lianc el terliam, tertium quartam, quartum quintam el sextam excipit. Costae tertiae, quartac et quiulae emineutio insidens. Ossa haec ommia sine ullo dubio pro sterni reptebris habenda suml; de reliquis, imo scapulae parte, ambigilul.

Primum, vertebram ex descriptis sternalibus anticam margine postico presse sequens, ejus margini antico capsula synoviali junctum, ounibus maximum, parte postica, impari, latiore sed breviore, et transrersa, longiore sed angustiore, ipsius fini antico imposila componitur, figuram ' $\Gamma$ referens. Et lujus facies inferior spimula notatur.

Sequitur par ossiculorum, omnibus hujus regionis longe minorum, longitudiualium, longorum, sed valde tenuium, parti ossis modo descripti transrersae archissime agglutinatorum, extremis internis sese fere tangentium, externis, crassioribns scapulac marginis anterioris eminentiac, acromio, capsnla satis laxa unilorum.

Par alterum, fere quadratum, longe brevius, at latius, inter rami transrersi ossis primi partem internam, ranum ejusdem lougitudinalem, cujus marginem, nomnihil eliam, praccipue in parte inferiore, faciem postcriorem legit, et scapulac marginis auterioris partem inlimam pouilur.

Margini ejus interno toti insidet lanima cartilaginea satis lata, (1umn lineam nbicue in majore lnabeat, candem oppositi lateris altingens. Scapnlac capsula synoviali longa, sed angusta, reliquis ossibus tela cellılosa laxa mmilıur.

Ut melius de ossium lnujns regionis natura dispntari possit, scapula qnoque describenda est. Oblonga est, antrorsum conrexa, retrorsum concara el mangine superiore, minore, anteriore el posteriore circumdatur. Pars longe milor, superior facie internat ex exterua, inferior anteriore et posteriore gaudet. Ubi junguntur, cavitas adest glenoidea, antrorsmm et retrorsm aperta. Margo auterior parlis superioris versus inferiora extrorsum primo flexus, dein eminculiann, acromion, antrorsm et introrsum rersam, emiltit. Ossis secundi extremmon exlenum excipit, extremo inferiore sterni reptebrac, quam diximus, primac mobili nexı, partis infra carmm glenoides posilae margine interno ossi terlio jungitur.

Marginis superioris extremo postico frustulum osseum, medio cartiligo plana, rotunduscula insidet.

In utroque scelelo, quod coram habeo, scapula, modo descripto, turicum est os, sed in regione cavi articnlaris restigia disjunctionis in actate minus provecta observandac saltem in masculo adsunt.

Jam, ad explicationem ossium descriptorum accedens, primo, me jam diu, et praecipue anno MIDCCCXXI, ubi ultima rice Parisiis degeban, adspectu sceletorum ad scntentian meam compulsum fuisse, dein me, quac censeo, ea modestia proferre, ut facilline argmmentis cedere contrariis paratus sim, moneo, quod lıoc in casu praccipue summus scriptorum dissensus suadet.

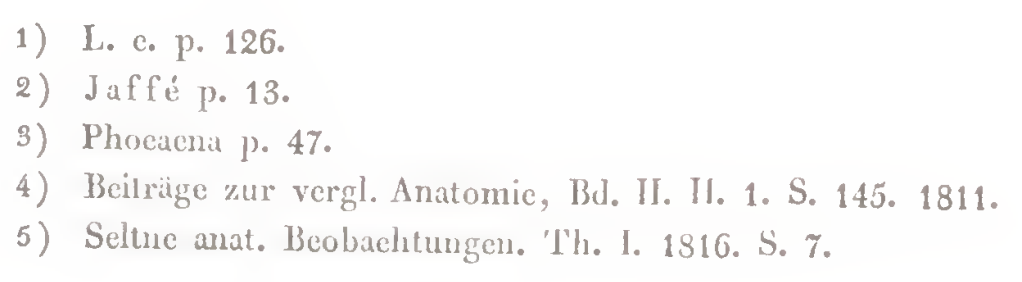


De scapulae significatione in unirersum minime dubilari potest, de parte ipsius inferiore vario modo judicatur.

Me judice 1) pars haec inferior processum coracoideum manmalinm, claviculam posteriorem s. coracoidcam arium atque amphibiorum; 2) os magnum, T forme, sterni partem supremam, minime autem, ut postca probabo, mambriun, sed os accessorium, uti in Phocis, manubrio appositum; 3) lamina ipsi imposita claviculam auteriorem, veram, s. acrounialem, sen furculan sistit. De pare secundo, quadrato dubilari potest, num ad scapulam, an ad stermm trahi debeat, equidem jam id 4) pro scapulae parte habco idque claviculae coracoideae anticae, partem scapulae inferiorem claviculac coracoideac posterioris nonine insigniendum esse censeo.

Et Mammalium et Avium, et Amphibiornm, presertim Sauriorum fabrica fortissime pro hac, ni fallor, stat sententia.

In Mammalibıs, ni fallor, omuibus 1) processus coracoideus nucleo proprio oritur, din a reliqua scapula distincto; 2) in Vespertilionibus maximus est et dcorsum atque introrsum flexus; 3) clavicula acronialis, ab acromio orla, nuncuan in linca nuclia cum opposita confluil; 4) in complumibus, pracipuc Celaceis nomullis, Phocis, Talpa, Myrmecophagis, Vespertilionibus adest cartilago vel os satis longum, ante costam primam a stcrui manubrio versus caput protensum, ossi nostro impari, primo loco descripto, quamr is longe mimıs sil, onuino comparandum; 5) ossis quadrati analoga exlibentur laminis osseis, faciei superiori processus coracoidei in homine juniore insidentibns; 6) os, quod milui clavicula est, cmm acromio et osse T forni jungitur, hoc autem scapulam non allingit, eodem prorsss modo clavicula mammalium acromio et sterno interponitur; 7) in Bradypo torquato observari fabricam, pulcherrime rem illustrantem. Duac revera adsunt clariculae, acrominalis et coracoidea, ejusdem fere magnitudiuis et satis longac, qunm in animali, pedem unum chun dimidio longo prima sex, haec octo lineas habeat. Acromialis ad processum coracoideum tendit, quen tangit, mininue extremun coracoideac posterius altingens; haec a processil coracoideo antrorsium et extrorsim usque ad steruun progreditur, miulune coun codem juncta.

Ares 1) claricula coracoidea gaudent, a scapula in unircrsum disjuncta quidem, sed synchondrosi firmissime cum eadem unita, stermun mobili nexu tangente. Struthiones omuino ad Ornithorhynchum et Monotremata in muiversum accedunt concrelione hujus ossis cmm relicpua scapula. 2) Stermum fere semper gaudel processu medio superiore, versus furculam, sire claviculam acromialem tendente, imo cum eadem confluente, in compluribus omnino eodem ac os $\mathrm{T}$ forme extremo superiore in ramos duos laterales abennte. 3) Furculae aviun dimidia fere semper quidem in linea mediana conflunnt, sed dantur exempla ejusdeu, quam lic descripsimus, et mammalia nec non amphibia semper exhibent, divisionis. Sic in Strige ulula et Ramphasto levirostri utrumque dimidimn nomnisi cartilagine unitum inveni. Semper etian furculac diutidia lateralia primo laterantur.

Naxiunum robur Sauriorum affert fabrica. In his 1) sternum omnino codem modo in dimidium anterius et posterius dehiscit, e quibms hoc os nostrum impar omnino refert, nisi quod ramms transversus brevior sit; 2) adest clavicula acromialis, ab opposita disjuncta, et longe major; 3) coracoidea codem modo cum sterno articulo juncta; 4) lamina cartilaginea quovis respectu cum osse nostro tertio comparanda, nec ad stermum pertinens, cui laxissime unitur, arcte claviculae coracoideac juncta.

Accedunt rationes, ex myologia petendac. Nutaloris capitis venter internus soli ossi T formi, (sterno) externus et pars cucullaris anterior soli claviculac adhacret. $\Lambda \mathrm{b}$ osse quadrato, pro clariculac coracoideac parte a me habito non solum musculus, deltoidis partem anticam referens, sed capnt longum, anterius bicipilis, musculus pectorali minori analogus, orilur. Processui coracoideo capnt bicipitis breve et coracobrachialis inliguntur.

Sic, ni fallor, argumentis satis firmis sententia mea fulcilur, co fortior, si, reliqua mínus cun natura conrenire, probare datum fuerit.

Cl. Ilomius, primus, qui de Monotrematum ostcologia egit, verbis nonnisi scapulam et os primum, impar indicarit, quamvis in sinistro saltem latere os tertium aduexum sit, scapulac vim necessario perspexil, os autem tertimn nonnisi pro ipsins parte habet, de impari quid sentiat, in dubio relinquens, sterno se idem haud admumerare clare iudicat, quum, sterno insidere, et processus ipsius transversos clavicularum oflicio fumgi, dicat ${ }^{1}$ ). Imo in figurarum explicatione totum omnino os claviculas

1) Ph. Tr. 1802. p. 75. 
aliorum animalium referre diserte dicil $\left.{ }^{1}\right)$. Nusquam autem Homium, uli cl. IIoevenius ${ }^{2}$ ) vult, scapulac processum inferiorem pro furcula Jabuisse invenio.

Ego, in opusculo, jam auno MI)CCCV. Parisiis elaborato, primus post cum, rerbis claviculam acromialem et partem coracoideac, seu par primum et secundum, linea quoque divisionis claviculam coracoideam ipsam iudicans, lanc pro avium clavicula coracoidea, primam cum osse impari pro clavicula mammalium, seu arium furcula habıi.

Cl. Tiedemannus, praccipuc Homianis fignris atque descriptionibus, forsan et propria, quum Parisiis degeret, inspectionc usus, os impar pro prima sterui parte se habere, ramos trausversos clavicularum vices gerere, et medio margini scapulac affigi, scapulan ranis duobns, anteriore, ad unionem crum osse sterni prino latescente, posteriore, sueto more versuss spinam dorsi adscendente, componi dicit. Hace cl. rini verba propria qumm sint ${ }^{3}$ ), miror, cl. Hoevenimm, em apophysin ossis imparis transtersan pro clavicula, apophysin scapulac pro arimm furcula habuisse dicere ${ }^{4}$ ).

Blainvillitus os impar ad stermum trahit, par primum claviculam acromialem, sccmodum costam primam, partem scapulac inferiorem processum coracoidcum rocat, recte ejusclem cum scapula reliqua confluxim docens.

Geoffroyus os impar firculam, par primum acromion, sccmudum partem stcmi, s. os episternale, partem scapulac inferiorem claviculam coracoileam autumat.

Catus os inpar el par primum, quod cum IIomio ignorat, pro parte sterni suprema, reliqua, e quibus nomnisi scapulam nosse videtur, simnl pro lac et (lavicula mammalium propria luabet ${ }^{5}$ ).

Okenius os impar maubbrim sterni, par primum (lavicnlam acromialem, secundum partem sterni, partem scapulae inferiorem claviculam coracoideam esse judicat ${ }^{6}$ ).

Rulo/phius, ultimo loco de hisce ossibus disscrens, uli ego dixeram, os impar pro furculae corpore, par primum pro jpsius epriphsi, contra par secundun et partem scapulac inferioren pro clavicula vera mammalium, in dıo dimidia disrupta Jabel, quorum illud claviculam anteriorem, hoc posteriorem vocat.

Quot capita, tot scnsus! Sic quum singuli, quanvis rarie, ossa hace cum aliis mimmalium coutulecint ossibus, cl. Iñoxium, ,in hodicrno scientiac statu se in manmalibus cormu analoga hand invenisse, fateri, ${ }^{6}$ miror ${ }^{7}$ ).

Equidem cum Blainvillio fere omnino couscntiens, nno praceipue puncto disscntio, nulla, par secundum pro prima costa esse Jabendum suadeute analogia et dissuadentibns, quac ex Mammalium et Sauriorum structura, nee non ex myologia exhibui, aronmentis.

Cl. Blainvillius variis quidem rationibus suam fulciri studet scntentiam, sed ni fallor, ex parte contra ipsum pugnantibus.

Primo, quum sccundum cl. Ilomium vasa axillaria per foramen inter scapulam, os quadrilaterum et os T forme excant, semper autem rasa axillaria circa costam priman flectantur, infert, jann lanc ob causam os quadrilaterum pro prima costa esse habendum. J)olendum autem, visa minime via, quam cl. anctor dicit, sed cum nervis sueto more inter scapulam el reram costam prinam incedere, ut sic hoc ipso argumento sententia refellatur.

Hunc autem errorem minime cl. Iomio, sed Gallo tantum interpreti esse tribueudum, facile ex utriusque vocium comparatione ingenua patet. Cl. enim Homius niluil de illa, quam cl. Blainvillius dicit, per foramen steruo-scapulare memorat via, sed os T forme cum scapula tegumentum osseum satis forte sistere asseril, sub quo colli vasa magna, compressioni sic laud obnoxia, progrediantur. Scnsum loci adducti ad stabiliendam opinionem propriam tam aperte corruptum esse patet, ut cl. virum famosi scriptoris de psychica organorum dignitate discipulum putes!

Cl. Tiedemanni voces: Scapulac processum anteriorem ita primi ossis stemalis margini laterali affigi, ut in tota hac thoracis parte adsit nomisi foramen pro rasorum transitu ${ }^{8}$ ) robur sententiae quam refutari dare possint, sed haud uno vilio laborant. Foramen enim hoc in sceleto quamris

\footnotetext{
1) Pl. Tr. 1802. p. 83.

2) L. c. P. 356.

3) Zool. B3. I. S. 586. 587.

4) L. c. p. 356.

5) Zootomic p. 164. 186.

6) Isis 1823. p. 445. 446.

7) Frorieps Nolizen 1823. S. 292

8) L. c. p. $58 \%$.
} 
adsit, minime tamen vasa, nisi inferioris ordinis, transmitlit et fere omnino, excepta parte superiore, minima, clandilur musculo, fuen pro deloidis parte anteriore me habere, jum supera dixi. Dein, ramus, quem dicil, el qui perera os proprium est, minime margini ossis sterni laterali affigitur, sed, eum transscenclens facici superiori incumbit, louge rectius jam antea disertis verbis cl. Homio monente, hanc parlen altero margine supponi ossi sterum pracedente ${ }^{1}$ ).

Alterum argumentum, inscrtio sc. primae carlilaginis costalis in manubrium solum, secundac inter idem et secundam sterui vertebram, hand magis probat. Nam primo equidem minime os T forme pro unaunbrio, sed pro parte accessoria habendun censeo. Angetur enim duplici modo sterni longitudo anteriora versuts, ant crescente manubrio, aut adjecta nova vertebra. Illud in Vespertilionibus, Talpis, Myrmecophagis, hoc in Phocis et Mouotrematibus obtiuet. Discrimen facile noscitur. Iu illo casu costa prima antico, in hoc secundo ossi inseritur.

Hoc el iu Phocis et iu Mouotrematibus locum habere, cl. Blainvillius quamvis contrarium vidisse videatur, quivis facile viderit. Sic igitur hoc quocpne argumentum coutra ipsius sententiam pugnat. Celcrim os quadrilaterum cum osse ' $\mathrm{T}$ formi nonnisi tela cellulosa laxissima unitur.

Contra sententiam, os impar pro furcula habentem, argumenta, quibus idem pro prima sterni parte esse habendum eviucere studui, pugnant. Quun autem clavicula acromialis furculam avium referat, argnmentis quoqne, pro primi paris significatione adductis, Jaec sententia corruit.

Jam, si os impar ad sterum pertiuct, nec furculam s. claviculam acromialem sistit, argumentorum, quae pro paris primi signilicatione adduxi, robur augetur. Accedit, quod et Geoffroyi et Rudolphii sententia aualogia omnino careat, quum neque acromion tantopere in ullo animali extendatur, neque furculac epipleyses dentur, contra Rudolphii igitur iuprimis sententiam pugnet inutilis plane nororum objectorum introdnctio, nomisi ubi omuino desunt, cum quibus comparentur, tentanda.

Par secundum quo miuus cum Geoffroyo pro sterni parte labean, dissuadet aretus ejusdem cum processu coracoideo et in Monotrematibus et in Sauries nexus, laxissimus in ultisque cum steruo anteriore s. osse nostro impari.

Cl. Rudolphius ,clariculam mammalium communem in dimidium posterius et anterius divisam esse prutat, quorum loc avium more cum scapula ad formaudum carum articulare pro lnumero coit, illud, accessorium ,claviculam dilatat amphibiorum more.“

Haec antem, ni fallor, facile refelli possunt. Mammalium cnim clavicula, quum nnnquam ad formandum carum hoc articulare couferat, hic parti minime conferri potest. Avium exemplum nihil probat, quum nostris dichus fere inter omues coustet, claviculam, quau dicunt, avium non mammalium claviculac, sed processui coracoideo respondere. Intic antem avinm claviculae, s. coracoideae, omnino convenit illud Oruithorlynchi os, quod cl. Rudolphins pro claviculae dimidio posteriore liabet. Hujus cum scapula et sterno unio mininte, quicquid dicat ct. vir, probat, cam non pro scapulae parte esse liabendam, nam scipulie ad avium typum producitur ad stermun usque uucrs, ad mam malium unitur cum relipua scapula.

Os impar, (nam de lıo sine dubio nomine claviculac, sc. furcularis, loquitur) dilatatur quidem osse quadrilatero, sed hine minime efficilur, os hoc pro claviculae communis parte antica esse habendum, quum practer argumenta jam supra adducta ejus situs, conjunctio et forma senteutiam liauc fortiter impugnent. Neutrum igitur horum ossium cum clavicula acromiali s. communi mammalinm quid commune habet, quamris os anlicum, contra cl. viri sententiam, cum sterro, i. e. osse $T$ formi et scapula, i. e. parte coracoidea, uniatur.

Accelit, quod detur vera clavicula acromialis, quod arguncntis probare studuimus, mera negationc haud refulsis.

Sic igitur Blainvillio, Caro et Olenio, respectu ossis imparis in universum, illis quoad sententiam de clavicula acromiali, accedo, cum Blainvillio, Geoffroyo et Okenio de clavicula s. processu coracoideo omuino conveuiens, ab omuibus respectu ossis tertii recedo.

In universum me cum cl. antecessoribus de osse impari convenire dixi, minime antem, cum cl. Caro id pro sterui manubrio, sed parte adscititia essc labendum, quum hoc os omnimo a sequente sit separatım, pro rero manubrio ob uniouem cum clavicula coracoidea el costa prima necessario habendo.

Quac protuli argumenta, praceipue accurata partiun descriptioue firmantur. Homius, Tiedemannus

1) Phil. 'Tr. 1802. p. 74. 
et Carus, hi nomisi Homii fignras sequentes, Carus meas, earmunne descriplionem jam diu anlecpnam scripsit, publici juris factas negligeus, claviculam acromialem et partem anticam coracoileac, quamvis separationis ipsius lineam el. Ilomii artifex depinxerit, practervidere. Sic vix rectum judiciun ferri el praecipne el. Carus facile in errores incidere potnil, quos, ni fallor, commisil. Primo claviculan distinctam desiderari (licit ${ }^{1}$ ), qumn et acromialis tola et coracoidene saltem pars ipsins omnino separata arlsit, dein el. Momium, qui, quanvis claviculan acromialem veram practerviderit, tamen saltem ossis 'T formis rammm transversumn pro ea hahnit, ila corrigit ${ }^{2}$ ), $u 1$ omuino eandem substituat sententiam, clavicula chim accessoria ranarmun, quacun cl. vir potius rammm illum conferendum censet, ommino clavicula manmalinm rema, s. acromialis est. Cl. Itoctenio anctore cl. Carus rammm transversum ossis 'T formis pro avium fincenla hahet, quod, quamvis haud disertis verbis el. vir dixeril, implicile tamen asseril, qumm fiurculam el clavicnlam ipsi dictam accessoriam recte pro eodem habeat $\left.{ }^{3}\right)$. Equidem claviculam acromialem, coracoideam, et os quadrilatermm, ipsi accedens descripsi et depinxi, et primi ossis sejunctionem adnolavi ${ }^{4}$ ), sed ossis imparis rammm transversum rlavicula acromiali a scapnla sejungi tunc temporis haud perspexi. Blainvillius emm nomisi fere ad scapulan usque perlingere, rectius momuit ${ }^{5}$ ). Limelolphius longe minus recte ossis imparis ramm transversum scapulae afligi disertis rerbis docel, qnod ommino naturae repugnat. Clariculam acromialem habet quidem, sed utrissque in medio disjunctionem quum repbis haud indicet, figura ommino negat, nnam tautum laminam exhibente ${ }^{6}$ ).

Nim actate provectione duo clariculac acromialis dimidia inter se et cum ramo ossis stemi primi

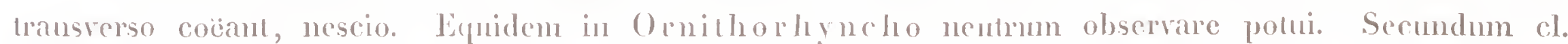
lindolphium in adultiore fere onmino cum hoc osse concrevil, sed maceralione forsan hic coalitus solvi potnisset. Nec, si hor: non successeril, quod eo facilins dederim, qunm ipse, quanvis in Onnithothyncho et Echidna selosa utrumpue os riderim separatum, in Echidna lamen loystrici concretionem ommino absolutan invencrin, inde patel, quid de hujns ossis vi eflici possit. Imo optime ad rem nostram facil concretio perfecta inter furenlan avium s. claviculam acromialem cum sterno, Grallis et Pahnipedibus nommullis solemnis. Clavicula acronialis fumm avimm furenla sit, ex muione dimidii utrinsque contra hujus ossis natmam eo mimus aliquid deduci possit, qumm in avibus furculae dimidia prima vitac periodo semper separata sint.

\section{ก. 11.}

Jam ad reliqua extremitatis anterioris ossa transimns.

Os humeri magnmm, praecipue litlissimum.

Capul, minime rotundum, partenn cjus altissimam sistil, conrexam, sed minime rotundam, valde oblongam, curvatam, collo nullo a reliquo osse scparatam. Excipilun retrorsum ab cminentia brevi, niquetra, epiplysin tantun referente, antrorsum a spina, ad medium usque os descendente. Dimidimn ossis superius extrorsmm convexum, introtsum ralde concarmm ab utroque latere, inferins sensu contrario compressum directione transversa dilatatum est.

In extremo inferiore caput rotnudum, ab ossibus anlibrachii simul exceptum, extrorsum et sur-

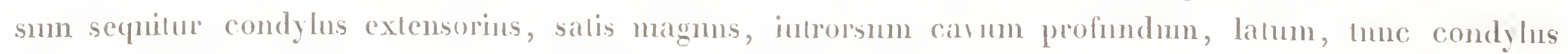
flexorins, major, cujus basi foramen adslat anplissimnm, non tantum anmalibns mansupialibus, quod montuil cl. Blaincillius ${ }^{7}$ ), sed multis aliis, praecipue Feris et Gliribus, nec non (Ouadrumanis, commme, munquan autem, quatenus equidem meis celoctns fini indagationibus, extra mammalimm classem inreniundum.

Ossa antibrachii, in codem plano posita, proxime distantia immobili nexu ita jumgmmtur, ut radius introrsum, ulna extrorsum spectet. Lurumque crassum, prismaticum, in extremis valde inflatum. Radins rectus, fere terlia parte ulnâ minor. Hujus olecranon longissimum, extremo latissimo, in uncum superiotem et inferiorem protracto, terminatnr. Os ntrumpue cavilate glenoidea communi, profmnda tmmeri caput inferins ila excipit, nt antibrachinm nomnisi extrorsum extendi, introrsum flecti

\footnotetext{
1) Zow.t. p. 185.

2) Hlid. p. 180 .

3) L. c. p. 151

4) I.. c. p. 80.

5) L. (. p. ? 9

6) L. c. p. 13. 'Tul. II. I. 3.

7) L. c. p. 13.
} 
queat. Facies radii inferior excarationes duas profundas, eminentia alta divisas, contra ulnae trochleam sistit, ommes in codem plino sitas.

Cappus brevissiuns duohns ossium parrorum ordinibus compouitur, quorum quivis quatuor coutiuct. In superiore ossiculum longinsculum, tripuetrum, musculare, nihil ad articulationem cum ossibus antibrachii faciens, maii extremo inferiore supponitur; sequitur maximum scaphoides, cum lnnari radio respondens; dein minus, planum, cum quarto, perpendiculari, pisiformi, satis magno uluae extremun ita recipiens, ul etiam pisiforme facie cartilaginea inferiorem, minorem articuli partem efficial, ulnam fulciens.

Ex Ordinis inferioris ossibus, scaptoide et triquetro minorihus, non autem parvis, tria interua tribus metacarpi ossibus, et parti radiali quati, quartum huic, minima ex parte et quinto uniuntur.

Os metacarpi quintmu uberculo retrorsum protracto, cartilagine tecto cavitate glenoidea ossis triquetri recipitur, (nod Blaincillius practervidil, nonnisi ejus uninis cam unciformi mentionem faciens ${ }^{2}$ ).

Ossa metacarpi et digitorum brevia, crassa, phatanx terlia plana, obtuse acuminata, longior reliquis, secunda brevissima. Ossa melacappi plualangibus longiora. Onartum et quintum longissima, sequitur primum, dein tertium, ultimo loco secundum.

\section{§. 12.}

Pelvis satis magua et ampla, praccipue in parte inferiore. Os ilium angustum, crassum, prismaticum, antrorsum convexum, retrorsun concarum, sursum dilatatur. Os ischii retrorsum in processum longum epiphysi parva terminatum extrahitur. In loco unionis ossis ilimm cum osse pubis eminentia ponitur ileo-pectinea louga et crassa, quan tubercuhun sequitur minus, sed idem crassum. Symplysis pubis longissima et latissima ossibus lit omnino in unum roncretis. Hoc in ulroque animali omnino codem modo sese hahet, neque ulhm pelvis utriusque invenire polui discrimen.

Ramo fere toli horizontali ossis pubis imponitur os, quod rocaut, marsupiale, longmm, praccipue in externo inferiore latissimum, extrorsum et antrorsum versum.

Acetabulum circa in media pelvis facie externa positum, salis profumdum perfecte osseum est, quum iu Echidna, avium more, fimdo osseo careat.

Forameu obturatorium maximum.

Os femoris humero minus, praesertim teunius et angustius. Caput rotundum, prominens, rectum, medinm inter trochanteres, externum et interumm ponitur, in lineam asperam exteruam, breviorem, et internam, ad medium usque femur excurreutes.

Recte cl. Rudolphinm ligamenti teretis defectum adnotasse video, hine caput omnino laeve, nusquam impressum.

Crus femore multo longius est. Ossa, praceipue in parte superiore mulum distant.

Tibia fere triplo crassior fibulì, sed quarti parte brevior, extrorsum ralde couvexa, introrsum concava. Fibula, recta, capitulo superiore facici externae condyli ossis femoris externi et tibiac simul communi capsula unitur, tunc uhac modo in processum altum, planm, extremo superiore dilatatum abit.

Patella magna, crassa, lata. In ligamentis genu, practer coufluxum capsulac magnae genu cum latcrali tibio-libulari in unum cavum, omnino nilhil insoliti vidi.

'Tarsus carpo duplo longior, sed augustior est, et octo ossibus componitur. Ex his tria primum ordinem ita constituunt, ut duo longe majoria, calcaneus et astragalus, fere ommem efficiant, tertium, ex olunibus minimum, nounisi accessorium sit.

Calcancus, maximus, fere quadratns, non pone et infra astragalum positus, parte externa, longe maxime et fibulaun, cum cujus parte externa articulatur, et ordinem secundum extrorsun transcendit. Astragalus, nounihil minor, planns, facie articulari globosa, exterua majore, cum calcauco fibulae, interna convexa, angustiore, tibiac faciei articulari inferiori respondel. Facies ipsins interna, excavata, capitulum tibiac extrorsum prominens recipit. Utriusque ossis cruralis extrema capsula propria includumitur.

Ossiculum primi ordinis tertium, in planta tantum conspicuum, rolnudum extremo anteriore facici astragali inferioris insidet.

1) L. c. p. 14. 
Ordo secundus, primo praesertim, modo jan dicto, longe angustior, sneto more quiuque ossibus, nihil insoliti offereutibus, componitur.

Ossium, quem descripsi, munerms et habitns nomuilil ab iis, quos Blaincillines et ego descripsere, differt. Equiden ${ }^{1}$ ) pridem in Echidna septem invenisse milhi visns, de Ornithorhyncho niltil momi. Contra Blaincillius octo, non antem meo modo, descripsil. Tertium ordinis primi, revera praesens, practervidit, contra sextmm in ordine secundo addil, ossi cuboidco atque metalarsi quinto ita interpositnu, ut hoc non, sueto nore cum cuboideo uniatnr ${ }^{2}$ ). Facilline ossiculum tertium primi ordinis practervideri potnisse fateor, quomodo autem os ultimo loco descriplum invenini potuerit, nisi falbrica ab eal, quam coram habeo, adfuerit omnino diversa, nescio. In mei salten sceleti utroque pede Inetatarsi quintum, nomnisi cum cuboide, satis magno articnlatur, nee, quamvis accuratissine dissecans, ullum ossiculi interpositi indicium invenire potui.

Ossa metatitrsi et phatanges exceptis pollicaribus, crassioribus, tenniora ossibus manus et magis directione trausversa compressa. Netatarsi ossa a pollice ad digitum quintum longitudine angentur, phalangibns nomilail majora. Hae ommes et per omnes digitos fere aequales, exceptis pollicaribus, brevioribus.

\section{§. 13.}

In capite cranium rotundusculum, antrorsun nonnihil angustatum, facies valde plana, antrorsum sensim magis depressa, simul valde dikatata. Latitudo lujjus pirtis valde angetur cartilagine, marginibus lateralibus fere tolis, et antico appositi, ad quatnor lineas lata, in ntroque litere ad duos pollices longa, de qua in describendo naso fusins agemus. Ossa, pracecipue cranii, ad arinm typum cilissime coalescunt, quun ne nuinimm quidem sulurae restiginm neque alii, neque ego invenerimms. Ceterum tennia sunt, sed firma el substintia tantum vilrea conflata.

Paries occipitalis teniter antrorsun adsceudit. Foramine occipitati gaudet maximo, recto, rotundo, sursun in angustiorem recessum abennte. Condyli occipitales maximi, fere transrersi, et extrenis internis in omnibns, quac vidi, craniis sese tangentibns. Spina occipilalis longindinalis parva. Facies superior plana, a laterali linea undulata, parum eminente, limite foveae temporalis, hand profundae superiore secernitur.

Hace et orbita, parum profunda, sursiun, antrorsiun et retrorsum aperta, ommino in umm confluunt. Zygoma rem, in nostro animale peaceipne attentione diguam, radicem sc. duas posteriores, snperiorem et inferiorem oflert, quac spatio duarum lincarun inter se distant ef cum cranio spatium nums lineae latitndinis inchudunt, forma, piscibus, atuphibiis, nec non interdum avibus solemuis. Ifoc non tautnm in ntroque, fnod mili ad mants est, sceleto, sed clian in craniis Parisinis ommibns vidi et Echidnarum craniorum inspectio hanc fibrican Monotrematun ordini communem esse docuit. Differt Echidna ab Oruithorhyncho nomisi latitudine ntriusque radicis longe majore. Foramen canalis hinc nati posterins jam in Echidna indicavi ${ }^{1}$ ), canalem ipsun ignorans.

Cavitas articularis pro maxilla inferiore transrersa, concilla, extrorsum, praceipne introrsnm et retrorsmon clausa, antrorsum iperta, maxima parte inlerna etian tympino eflici videtur, quod, quamvis hoc os cum religuo cratio concreverit, propter ossis quadrati vim atlentione dignissimnn est. Zygoma lougun, altum, rechnu. In ficcie cranii inferiore, cujus pars anterior, ut videtur, ossibus palati longe protractis, tegitur, praccipue planities et latitudo corporis ossis occipilis et magnitndo foranuinmm lacerorum, posteriorum, hic rolundormu, ipsum et condylos intercedentimu, notanta. Maxima ex parte explentur membrana librosa, forti et capsula occipito-atlantali communi inchuduntur.

liacies tan plaua est, ut paries tantum superior et inferior et margines laterales descrihi possint. Paries superior paullulum ante mediun longitndinem fortmine antrorsum maxime dilatato delusseit, et in ramos duos secedil, divergentes nomnisi extremis anticis, introrsmu quac flectuntur, convergentes. Facies inferior, vel palatum, modice concavum, longissimmn, hine nares posteriores partun a foramine occipitali distant. Ad ipsius narginem lateralem versus extremum posterins lamina ponitur longitndinalis, horizontalis, angusta, extremo postico extrorsum flexa, sine dubio processus pterygoideus internus,

1) L. с. 1. $7 \% .82$

2) L. c. p. 16. 17.

8) Beitr. 'Tuab. 9. 'rig. 1. 
quem cum cl. Rudolptio in utroque specimine omnino mobilem inveni. Tune subilo dilatatur palatum, dentes maxillares ferens superiores.

Denuo per nonnullarum linearmu spatimn contractum, antrorsmm, uli modo monuimus, dilata111. Apertura inferior superiore tertia fere parte brevior est, protensis in spinam maxillae superioris ramis palatinis.

Dimimitur etiam magis in parte media ossiculo, luic spinac insidente, extremo postico et antico dilatato, el 8 forman referente, sursmm in dinidio majore posteriori in processmm longitudinalem sulcatum protenso, palati ossei longitudinem augente et nasi carnu intrante.

In faciei pariete superiore el inferiore maxima, ramos neri supramaxillaris culaneos emittentia adsumt foramina. Posticum laterale, in faciei orbitae anterioris parle inferiore positum, canalis infravel pracorbitalis initium est. In margine laterali, quinque circiler lineas ante dentem molarem primum cornm, quibns rani, in faciei cute distribnendi exemnt, versus finem hujus marginis secundum, minns, adest. Tertimn inveni in medio circiter margine laterali aperturae narimn. His minora duo in palato offendi, palalinum posterius minns, incisirmm, anticmm, majns.

Practerea in facie superiore, quatnor circiler lineas ante orbitan, aliud, salis magnum, adest, nervum ethmoidalem quinti, hic valde magnum, emiltens. Per hoc et tertimn laterate nervis ethmoidalis, hic maximns, in faciei cutem tendit. Blainrillius primum recte in foream ellmoidalem abire dicit, hoc ommino, quatenus nervi iter sit, practervidit ${ }^{1}$ ). Riudolphius ommino nilil de ulroque habet, quamvis primum artifex dederit.

Et faciei ossa fere omnino concrevere, 11 parmm tantum distingni possint.

In specimine masculo, quod ob oculos luabeo, nonnisi ossicula, incisiva inferiora et superiora reliquis toto ambitu sejuncta sullt. Practerea adest rimula mceliana fere semipollicaris, ossa, ut videtı1', nomnisi nasi sejungens. Haec in specimine a Rludolphio descripto usque ad os fiontis, per omnem ossium nasalium satis magnorum, longitudiuem protendebatur ${ }^{2}$ ). Praeterea et ossa ungnis et palalina a reliquis segregata erant.

Concretis omuibus fere faciei ossibus, forma singulorum minime accurate describi polest.

Patet tanen ex descriptione et figura c. Rudolphii, minns bene Blainillium ossa nasi minimis Lantum stylis comparasse. Ossa intermaxillaria inferiora idem anctor minime delunivit, sed cl. Rudolphius recte cadem pro parte ossium incisirornm habnit. Partem internam ab externa, rostri dilatatione separatan, eadem sistere pulat, recte, si partem palatinam, vel inferiorem dicere rohnit. Eodem omnino modo in Bradypode didactylo dispupta sumt, dinidio tamen superiore carmm uarinm superne tegente. In Bradypode tridactylo os incisinum nomisi (nooad partem palatinam superstes, fere omuino incisirım Oruithorhytuchi palatinum sese habel.

Maxilla inferior longitucline fere capul superius aequat, sed, praceipue in dimidio anteriore longe angustior est. Valde huntilis, in triente anteriore extrorsmn flectilur et dilatatur, ut facie jam gaudeat superiore et inferiore, et dinidinn dextrum a sinistro tribns lineis distet. Ubi sic inflecti atque in planum horizontale abire incipit, per trimm linearum spatimm temi et molli cartilagine nexu laxissimo bina dimidia mminntur. Spatimn, inter extrema antica positum primo nomnisi temui oris membrana et cellulosa expletur. Extremo anteriori ipsi apponilur cartilago semilunaris, duas lineas, in parte media, inter extrema penetrante etian quatuor lala et labinn inferius hac parte fnlciens. Ranums adscendens vix adscendit, et condylo maximo, transrerso, convexo teminatur. Processus coronoidens brevissimus, fere mullus, infra ipsum in facie externa carnm coccum, duas limeas latum el alınn, ad quatuor longun antrorsum directum, musculi temporalis extremo anteriore repletum. Processulo coronoideo in facie interna opponitur alins, major, sursum retrorsumque direchus, musculo plerygoideo interno fulcrum prachens.

Mox ante hnuc maxilla pro dente molari dilatatur, dein contrahitur, mox demo expanditur, margine externo dentem anteriorem excipiens. Foraminibns ramo inframaxillari, nervi divisi, maximo, respondentihns, maxilla gandet quatnor. Posticum, intemum, canalis alveolaris initinn, presse processum ptergoidcum sequitur, antica, quibus nerri rami cxemnt, tria adsunt, primmm, maximnm ante et infra dentem molarem in facie externa positum; secundum, minimum in media synchondrosi in facie in-

1) L. c. p. 21.

8) I. c. 'Till. 2. Tig. 2. 
tema incipiens et in sulcum subito abiens ad extremum maxillac anticum contimualum; tertium, primo lraud multo minus, in facie extema, exacte ante synchoudrosin positum, sulco majore ad finem maxillac tendens.

Articnlo tempori-maxillari mullun rartilaginis interarticularis incst vestigium.

Aperti cranii facies interna cum externa apprime convenit. Ren maxime memorabilem, falcem osseam, jam cl. Blumenbachius invenit atque depingi curavit. Per totum verticem a fronte ad medimm occipnt decurens, in medio altissima, ad quatnor lincas habet. Nargo inferior, rectus, crassior, praceipue in extremo postico, ubi in mare, non antem in femina, sulcis et eminentiis transrersis cerebellaribus notatur. Panllo ante medium in mare foraminulo pertunditur, non omnilsus communi, quinm in femina liand adsil.

In Echidna nomisi leve lujus fabricae indicimm inveni.

Blumcnbachius ${ }^{1}$ ) el Ilomius ${ }^{2}$ ) hane formam illi, quam ares nomnullac offerunt, recte coutulere. Monet quidem cl. Blainvillius ${ }^{3}$ ), eam non ommibus avibus convenire, nee non in homine inlerdum obscrrari, et equidem jam dudum frequenliam ossescentiae in processu falciformi humano forsan fahrirae animalinm imitationem esse putavi ${ }^{4}$ ), sed rectins cum aliorum animalium statu normali, quam pathologico Ornithorhynchi fabrican comparari putem. Cetermm in aliis quoque mammalibus, praccipue Monodonte, Delphinis, Plocis, Trichecho, nec non Solipedibus, tales processus osseos falciformes inveniri, neminem fingil.

Cl. antem Ilomius minine, ut cl. Blamillius intellexisse videlur, hac falnica Ornithorhynchnm omnibns aribus accedere, sed disertis rerbis, cam nulli mammalimn, sed, (puamvis minori gradı nommollis aribns, sc. Platalea et Psittacis convenire, dixit. Eodem modo cl. Bhmmenbachius eam nommllis avibns, v. g. Telraoni trogallo adscripsil. Lquidem eam frequentins in avium classe reperi.

Ossicula morbose praccipue processui falciformi adnata cetermo a modo dictis ideo omnino diffe-. runt, quod extra processum ponantur, haec contra als ejus limina ntraque, in Ornithorhyncho nequidem cmm ca juncta involvantur.

Eo Ornithorhyolus cum aribus a mammalibus in unirersum differt, quod cun falce ossea maxima tentorii ossei nullum adsit indicinm.

In basi cranii simile quid ac in rertice occurrit; eleratio sc. media, quamris lnmilior et latior, a foranine occipitali ad sellam turcican, quâ terminatur, decnrrens. Sellac ipsilus, angnstae, paries posterior, altissimns in spinas laterales duas, maximas acmminatas, quarum sinistra dextram bis magnitudine vincit, excmrrit. Facies sellac angusta, sulcum longitudinalem profundum refert, carotidis riam. Pars ossis tempormun superior, supra pormun acusticun internum posita, quadrilatera, foveam profundam pro cerchelli Iobulo extino contince. Practer foranima procondyloidea jam supra dicta praceipre foranen ovale maximnn, rolumdım panllo mints et tertium, reliquis mints, sed satis magntum, foramen opticum fissurae orbitariae milum sistens, attentione digna sunt. Fovea cllmoidalis profimda, crista parra disisa, lamina angusta, parum rribrosa finimr.

\footnotetext{
1) Handb. der vergl. Auat. \$. 211.

2) Phil. 'Tr. 1800. p. 435. 1802. p. Ti

s) L. c. p. 18

4) Cuvier's Vorles, wh. vergl. Anal, ubers, ron Mleckel. 11. p. 182. 1809
} 


\section{§. 14.}

Musculorum systema, fere omuino nemini hucusque descriptum, eo lubentior accuratiori subjeci examini, quod complura, mimus solita et uuiversae myologiae comparatae, nimis neglectac, lucem afferentia, obtulit. Primo musculum cutaueum, dein reliquos musculos exlibebo, sic plysiologice agens.

\section{§. 15.}

Pamniculum carnosum recte cl. Itomius crassissimum dixit. Cadit hoc saltem in partem ejus maximam, inferiorem, thoracem, abdomen et dimidiun extrenitatis inferioris circundantem. Hujns crassities ad minimun linean aequat. Sursum seusim allenuatur, ut in cranio evanescat.

Decursus ipsius, quem neque IIomius, neque reliqui scriptores teligere, luic est.

Cingit fere totum corpus, truncum, excepta cauda, cranium, brachium et femur.

Cıtis faciei iuternae, magis etiam quau in aliis animalibus, arctissime aclhaeret tela cellulosa brevi, maxime stipata, ut nomnisi sumno negotio fasciculi musculares nilide praeparentur.

Contra, praecipue ubi thoraci et abdomini, brachio et femori respondet, tela cellulosa laxissina, copiosissima et adipe satis repleta ipsi musculisque subjaceutibus interponitur, ut ab his facillime separetur. Hinc ipse cum cute partibus subjacentibus longe major est, plicas quovis sensu satis magnas format et hinc inde gliscit.

Fibrac ipsius maxima ex parte sc. quatemus thoracem, abdomen et extremitates tegit, secundum corporis longitudinem decurrum. Satis subito tamen, iu colli fine inferiore directio fibrarun in transversam mutatur, quasi partium inferiorum moles transversas in longitudinem diduxisset, aut deficiente lac in superioribus dilatatione hic e longitudinalibus transversae fuerint factae.

Maxima ex parte musculus partibus subjacentibus lıaud affigitur. Jungitur tamen vertebris candalibus superioribus et nounullis artuum ossibns.

Extremum posterius obtusum fasciculis tribus vel quatuor crassis, quorum superiores inferioribus teguntur, a processuum transversorum vertebrarum caudalium anteriorum facie dorsali orilur. In caudae partis anticae facie superiori fasciculi utriusque lateris angulo obtuso coemut. Versus faciem abdominalem progrediens musculus cutaneus femore atque cruris parte suprema foramine oblongo, pollicem longitudine aequante, ita perforatur, ut parte posteriore et superiore in reliquan ipsins expansionem coutinua, popliti fasciculus sex circiter liueas aequans supponatur. Margo auterior iuferior nomilnil excavatur, infra symphysin ossinun pubis directione transrersa decurvit.

Supra medium lnme marginem faciei ipsins interuae spatio fere dimidii pollicis arclissime adliacret musculus transversus, ab ntriusque tibiae supremae facie flexoria ortus, ab ntroque latere in liuea mediana in unum confluens. Hlic, si duos pro umo habueris, ad tres pollices longus, tres ad quatuor lineas alıs, ceterum tenuis, fortiter crus cruri adducit, sic in natatione utilissimus.

lu cubili regione denuo simili foramine, sed ampliore finditur musculus, dein haud interruptus ad cranimu pergit.

In thorace supremo, e regione fissurae brachialis modo dictae, duobus fasciculis, quorum praecipue inferior, et decursm et adlacsione presse superiorem sequens, forlissinms est, tendinibus brevibus, musculo pectorali magno jumctis affigitur ossis humeri cristae anterioris diniclio inferiori.

Magis antrorsum atque introrsum, in ipso extremo thoracis anteriore e claviculae regione, simiTiter in plina duo, alterum exterunu, communis musculi continuationem, alterum inlermum, profundum finditur. Hoc fasciculum sistit pyranidalem, pollices duos longum, inferiore extremo quatuor, superiore duas lineas latum in regione ossis hyoidis cum musculi omo- et sternohyoidei strato superficiali junctum. Ex loc conjunctionis loco fasciculus hic, denuo dilatatus, extrorsum et antrorsum tendit, maxillam inferiorem transgreditur, et in maguum tendinum tenuissimorum numerum finditur, labii inferioris duobus trientibus posterioribus iusertorum. Flisce tendinibus labimn loc valide detrilnitur.

Fasciculus lic inferior Santoriniano conferendus videtur.

Stratum externum sensim, ut jan diximns, maxime attenuatum, subito fibris transversis com- 
positum collum et cranium laxe, pressius tamen parte reliqua circumdat, foraminibus ad aures oculosque ducentibus perforatum.

§. 16 .

Ex reliquis musculis primo eos, qui truncum componunt, et inter ipsos primo dorsales describendi veniunt.

§. $1 \%$.

Cucullaris in duos, ni graviter fallor, dividitur musculos. Alter, inferior, triangulum refert valde oblonguu atque tenuem, rertebra dorsali et costa decima et undecima, tendine lato oritur, dimidio inferiore latissimum tegens, sursum tendit atque tendine brevi, forti scapulae inseritur marginis snperioris fini antico.

Alter, superior, brevior, sed latior, quadrangularis, margine inferiore toto, posterioris dimidio inferiore oritur a claviculae acromialis dimidlio externo et scapulae margine anteriore et superiore. Hinc cum cognonnine confluens ad ligamentum nuclace et occiput adscendit, cui inseritur tendine tenui et augusto.

Ille scapulam retrorsum et deorsum, lic caput retrorsum traluit, faciem maxime erigens.

Latissimus dorsi, musculus longissimus et latissimus, a processibus spinosis vertebrarum dorsalium lumbariunque omnium, nec non costarum inferiorum undecim parte posteriori et media ortus, ad dinnidium marginis uluaris ossis lumneri inferins teudit, cui tendiue lato, crasso et forti, inscritur.

In parte anteriore crassissimus, quasi duplex, in stratun profundmu et superficiale dividitur.

Brachinu fortissime deorsum et retrorsum tralit.

Rhomboideus, unus tantum sed crassus, valde longus, cucullari tamen tenuior, a margiuis scapulae superioris dimidio inferiore ad occipnt tendit, a cucullari tectus spatio satis auplo inseritur.

Fortiter vel caput retrorsum vel scapulam antrorsum et introrsum trahit.

Levator scapulae in duos divisus videtur. Alter, posterior, longior, sed angustior, a margine scapulae anteriore, angulo superiore, et lauinula cartilawinea, medio margini scapulae superiori insidente ortus, satis crassus, antrorsum adscendit et atlantis processui trausverso inscritur.

Alter, crassior, anterior medio tricute marginis scapulae superioris ab hoc separatus, a triente hujus marginis anteriore ortus rectius adscendit, et, ante alterum, a quo omnino separatus est, allauti inseritur.

Ille collum et caput retrorsum, hic magis deorsum trahit. Uterque scapulam elevat et antrorsum movet.

Serratus anticus in musculos duos, omuino separatos, secessit.

Horum superior, fere quadratus, sex fascicnlis vertebris colli inferioribus quinque et dorsi primae inseritur, a margine scapulae postico ortum ducens.

Inferior, mnlto longior, sed angustior, triangularis ab apice scapulac inferiore fasciculis tribus ad costas tres proficiscens, versus extremum auterius faciei carum externae inseritur.

Splenius, micus, longissimus, collum fere totum explet, ortus a tendine intermedio quo cum opposito confluit, per totam cervicis longitudinem et processui mastoideo inseritur.

Birenter et complexus per totun decursum separati suut; complexus, longe crassior et latior, denuo in exterum et internum dividitur, quorıu ille simul profundus, brevior, hic superficialis, longior. Nullus inscriptionibus tendineis insignitur.

A suprenis vertebris dorsalibus et inferioribus cervicalibus ad occiput tendunt.

Opisthotenar, s. sacrolumbalis et longissimus dorsi in duos divisi sunt musculos, omnino separatos.

Sacrolumbalis, musculus latissimus, sed tenuis, ventre paullo crassiore a cristac ossis illium extremo posteriore ortus, costas omnes transscendit, ita tamen, ut singulis inligatur, cum fasciculis 
musculi cntanci, cucullaris inferioris, nec non latissimi dorsi, ab omnibus tecms, ubi costis inhacel, confhit, atque reptobarmu colli inferiorm quatuor vel quinque processibus transsersis inseritur.

Ob Ialitudinem el lennitatem extermm quasi mnsculorum intercostalium stratum referre dici potest.

Longissimus dorsi, multo crassior, sed angustior, a facic ossis sacri posteriore longe spinam ad tertian vel quarlam colli vertebram tendit.

Ilic musculus quum omnino a Jongissimo dorsi scjumgalur, contra cum spiuali, scmispinali, et multifido in unum omnino confluxit. Sistunt cnim musculum simplicissimmm, fasciculis obliquis, ex proressibus transversis inferioribus ad processus spinales superiores tendentibus compositnm.

Transversalis cerricis et trachelomastoideus omnino unmm sistunt muscuhm oblongum, a vertebris dorsalibus supremis ad processus transversos vertebrarum cervicalinm inferiores sex, et rentre superiore, longo, ad processum mastoideum teudentem.

Musculi quatuor capitis breves posteriores ralde longi et practer reclum inferiorem, potius tentucm, et valde extrorsum positum, crassissini sunt.

Obliquus inferior non tantum a processu spinoso epistrophaci, sed et terliae quartacque rertehrae cervicalis oritur. Supcrioris, omnimo quadrangularis, hasis margo totus anterior atlantis est.

R.cetus capilis lateralis minor, sed ratione tolins corporis satis magnus est.

Intertransversarios colli fortissimos obscrvari.

Levatores costarum fortissimi sunt et directione gandent haud ralde obliqua, exceptis tamen superioribus.

Intercostales nihil iusoliti exlibent. Crassissimi sunt, et superiores, ob spatium, costas superiores intercedens, satis latmm, latissimi, reliqui tamen eadem ratione tenuiores sunt.

Scaleni, forsan et pectoralis minor, imo et subclarius, duobus, ni gravissime fallor, referuntur unusculis, parvis, longitudinalibus, planis, superiore et inferiore, vicinissimis.

Supcrior, major a sterni manubrio ortus oblique extrorsum ad facici internac claviculac coracoideac quadratae rel auterioris partem internam posticam adscendit.

Eodem tractu, sed a primae costac cartilagine inferior, minor ad claviculac ejusdem coracoideae faciem internam decurrit. Uterque partem scapularem deprimil, decursu igitur el actione pectoralem minorem sistit.

Infra hosce musculos, claviculasque coracoideas, ipsis costacque supremac interposita nervi et vasa brachialia thorace exeunt.

Ne quis putet, me, et subclavium Tue trahentern, sententiam mean de osse quadrato coracoideo ipsum impugnare, monco, in plurimis animalibus, chiam mammalibus, subclitium el a scapula et quiden processu coracoideo oriri.

Musculi caudales haud ralde fortes smmt.

Faciei inferioris situm externum tenct brevis, ah ossis pubis ramo inferiore et tuhere ischii orlus, pudendo-caudalis, ad apophyses transversas rertebrarmu candalium anteriormm quinque tendens. Simul extremmm ipsins inferins deorsun flexum et ralde dilatatum in sphincteren cloacac ralde rohustum et fere pollicem longun mutatur.

Hunc unusculum introrsum sequitur longe major, cognomincm in linea mediana tangens, faciem anteriorem ossis sacri el vertcharmu candalimn tegens, et fasciculis parmm distinclis, oblique a processihns trausversis anteriorihus ad processus spinosos posteriores retrorsum et introrsum tendentibus composins, facie inferiore, libera ommino tendincus.

In facic superiore fortissinus, lunic similis, ab osse sacro et infina ossis ilimm parte ad rerteInam candalem ultimam descendit usque ad dimidiam caudan in dnos rentres facillime dividendus, 
internum et extermum. Fasciculi rentris interui processibus caudalibus spinosis posterioribus, externi transversis inseruntur.

Practerea in sulco a rertebrarum processibns spinalibus posterioribus et obliquis in utroque latere formato musculus alins, spinali respondens, rechss, satis crassus, decurrit.

Tn facie trunci anteriore primo musculi abdominales, amplissimi describendi reniunt.

Remoto musculo culanco primo, parte suprema, a pectorali majore techis apparet musculus abdominis externus s. oblique descendens. Spatium valde amplum explens, thoracem maxima ex parte, abdomen tomun a latere et facic anteriore tegens, a costis vertobralibus omuibus, excepta prima, ossis iliun extremo superiore, ossi pubis, extremo ossis marsupialis externo, libcro oritur. Margo iulcruns in tendiuem abit satis latum, margiue inferiore ossis marsupialis margini superiori insertum, margine interno, pectorali infero tecto, in linea mediana cognomini unitum. Annuli ingninalis vestigilun rullinm.

Obliquns internus s. adscendens s. medius externo longe minor, tenuissimus, praesertim in parte anteriore a crista ossis ilimm oriundus, fibris ommino rectis ad costarm septem inferiorum carlilagines adscendit. In tendinem anteriorem abiisse minime visns est, sed margine antico, acuto, bene distincto pollicis fere distantia a linea mediana liniri.

Transversus longe major, el nomuilil crassior ilidem ab osse ilium orlus, rertebrarum lumbalium processibus transversis et cartilaginum costalinm inferiorum undecim faciei internae inseritne. Fibris, ut nomen indicit, ommino transversis decurvit. 'Tendo luculentus, anplus, fortis, pone pyramidalen et rectum ad linean albam cum tendinis externi et cognominis oppositi fibris formandam abit.

Pyramidalis, alias ratione reliquorum abdomiuis mosculorum nec non totius corporis minimus, in Ornithorlynclo ad maximos pertinet, quum obliquus exterms quinta tantum parte ipsum rincat, rectus, maximus, nomnisi duplicem ejus sistat longitudinem.

Triangularis oriur margiue minino, inferiore a margine superiore ossis marsupialis, margine interno, recto lineam medianam langens ad sterni extremum inferius usque pertinet, eidem tamen luatul insertus.

Fortiter abdomen directione longitudinali angustat, ad costas deprimendas facit, motu autem con1rario os marsupiale antrorsum trahit.

Musculus hic sine dubio vim a me tribulam habet, qunm et situs tum in parte abdominis posteriore, tum ratione recti, cujns faciem inferiorem tegit, et forma triangularis lutic sententiae maxime fitreant.

Accedit defectus musculi pramidalis alins.

Ortus cjus ah osse marsupiali primo intuitu eandem hand suadere videretur, re autem propius cxaminala, el loc argmoneutum pro ea affereudum esse patel. Os enim marsupiale nonuisi partem esse ossis pubis, et quidem illam regionem, quae tubereulo respondet, jam inscrlio reliquormm abdominis musculorum, praceipue obliqui externi suadet. Accedit cl. Béclardi observatio nuclei ossei proprii, in lace regione in homine, praesertim in femina, uommequm observandi, interdum per omnem vilam mobili nexu reliepno ossi juncti, quem iugeruiosissime pro ossis marsupialis rudimento habet ${ }^{1}$ ).

Sic igitur musculus marsupialis a scriptoribus pro musculo proprio luabitus, ul parles tales bene mulac ex classe organorum, nomnisi nonuullis animalibus conrenieutum, eo rectius denominandus est, quod iu aumalibus marsupialibus reliquis, quae examinare contigit, sc. in Kanguro maximo, et nonnullis Didelplidum speciolnus, idem omnino rerum slatus observalur.

Rectus, longissinus, angustus quidem, sed crassus, fere tolam abdominis et thoracis longitudinem explet.

Pyramidali paullo angustior oritur ab ossis marsupialis margine postico et cartilaginis costae primac extremo antico, praecipue antem ossis sterui primi, extremo inferiori, nec non fere dimidio externo claviculae coracoideae posteriori, hasilari, fine supremo inserilur. Omunim Ornithorhynchi musculorum longissimus est. Inscriptionibus tendineis land distinguitur, sed firmiter faciei superiori tendinis obliqui externi ubique nectitur.

Diapluagma, maximum, parte lumbali fortissimo a vertebris dorsalibus inferioribus quatuor et lumbali prima oritur et late expansum, cartilaginibus undecim costarum inferiorum, excepta ultima, nec

1) Ueber die Osteose. M cekels Archiv fo die Physiologie. Bd. 6. p. 437. 
non sterni rertebrae infimac inseritur. Centrum tendineum ab Ilomio parvum nominari, miror, quum solita gaudeat ratione.

Nusaror capilis in utroque latere adest duplex, alter ab altero omuino separatus, alter superficialis, profumdus alter, lougissimus nuerque.

Ille, satis latus, hoc, angustissimo, longe latior ab extremo superiore rani longitudinalis ossis sterni primi et claviculae acronialis, hic ex parte media oritur. Uter(fue extremis omnino separatis ossi temporum pone aurem inseritur. Caput deorsum traluut.

Rectus capitis, longus maxinus, tolius colli longitudinem explet.

Lougus colli major ctian et fortior, at processibus spinosis anterioribus et facie inferiore corporum vertebrarum colli ounimm et quatuor dorsalium superiorum oblipue ad processus transversos cervicales omues interiores tendil.

\section{§. 18.}

Ex artus auterioris musculis Pectoralis major, revera maximns, praccipue longissimus est, triangularis, a clavicula acrouniali et primi ossis sternalis ramo transverso fere ad syuphysin ossium pubis extensus, fere totam thoracis abdoninisfue longitudinem explet. Margine interno cum opposito conlluit, praterea dinidio interiore, minore a costarum sternalinm anteriorum sex fine interno ortus, angulo superiore et externo toti ossis brachii spinae anticae inseritur tendine lato et brevi, hic simul cum musculo cutaneo unitus.

Iufra pectoralis partem anteriorem parvus ponitur musculus, quem pro deltoidis parte antica habuerim, ab ossis coracoidei quadrati extremo autico extrorsmn, ad summam ossis brachii cristam auticam descendens.

Extrorsum tres sequuntur musculi, directione opposita a scapulac facie externa ad ossis humeri cxtremum superius fere rectâ desceudeutes.

Ex lis primus, anticus, minimus, sine dubio suplospinatus, a praecedente, longe majore, tectus ab acromii facie inferiore ad capitis ossis lumeri basin tendit, fortiter os attollens. Sccundus, infraspiuatus, medius, longe major a media scapulac facie exterua tuberi ossis humeri antico inseritur intra et extrorsum a nodo dicto. Tertius, poue et magis etian extrorsum positus, reliquis longior et crassior, a scapulae apice supreno oblique iutrorsum et retrorsum ad lumeri tuberis antici diundium inferius abit. Cum secundo brachium extrorsum ducit et attollit, sine dubio deltoidis pars posterior.

Hunc musculum, ommino ab eodem separati, dno alii, in externa scapulac facic positi, sequmutnr, salis fortes.

Superficialis, posterior, longior, sed angustior, a marginis superioris scapulae parte posteriore ortus, ad mediun marginem interum ossis lumeri teudit, panllo ante latissinum dorsi ipsi iusertus. Sine duhio partem lujus musculi scapularem refert, omuino a reliquo et quoad insertionem separatam. Alter, ab eo tectus, a dimidio posteriore scapulac superficiei externac ortus, ante praecedentem ad faciem posticam ossis humeri tendit, infra caput ei insertum. Hic teres major est.

Uterque brachiom fortiter retrorsum et deorsum trahens, natatui maxime proficit.

Magis introrsum et deorsum tres alii poumntm musculi, ab aulibrachii flexoribns tecti, adductores el depressores lumeri. Uterque a parte scapulac coracoidea ad hmmeri faciem interuam dirigimr. Anterior, longe major, transversus, et a partis scapulae coracoideae facie interua fere tolat, nec non ab osse quadrato, infra cavitatem scapulac glenoideam extrorsum tendit et infra lumeri caput foreac profundissimae in ipsius facie posteriore poue latissimi dorsi tendinem inseritur. Ilic, ni fallor, aut teres minor, aut, quod rectins duxerim, coraco-brachialis superior, cst. Musculus alter, posterior, certo coracobrachialis, longior, sed teunissimms, ex parte coracoidea extremo inferiore tendine angusto ortus, ante summmm latissimum decurrens, infra cundem ossis brachii faciei anticae, paullulun supra condylum flexorium jungitur. Tertus, subscapularis, scapulate partem superiorem occupat, ab ipsins facie interna ad ossiculum, summo tuberi interno ossis lumneri nomisi capsulis junctum tendit.

Sequuntur musculi, aulibrachinm morentes. Duo adsunt flexores.

Margini musculi, supra secundo loco descripti, el pro deltoide antico habili, intimo apponitur musculus longe temuior, sed lougior, ex parte intima faciei exteruac ossis concoidei majoris, anterioris 
rersus ipsins extrenum iuferius, orimdus. Decurvit iufra cristam ossis brachii pectoralem et tendine terete inseritur radii superficiei flexoriae circiter in media ipsius ab utroque extremo distantia.

Jufra lunc musculum ah imo extremo iuferiore atche externo claviculae coracoideae posterioris, hic cum coracobrachiali sneto more arelissime junctus, oritur musculus decuplo crassior, sed brevior, extremo anteriore recti abdominis ab codem separatus. Iufra ipsum decurrit, tendo ipsins externus et inferior tendini ejusdem unitus radio circiler medio inscritur, ut nter'que num revera sistat musculum, bicipitem, qui et decursu et actione bicipitem hominis nonuullorumque mammalinm refert.

Tendine pectoralis majoris omnino ab hisce musculis discretus ab osse lumeri ad autibrachium decurrit Brachiens internus. Crassus et brevis orilur a facici ossis humeri externae dimidio iuferiore el presse supra tendinem bicipitis inscritnr lato line radii, minime autem nlnae, faciei internac. Hanc dinnidio snperiori, fere totins brachiei insertionem in compluribus mammalibus fieri, mox in anatomia mea comparata probabo.

Fortiter autibrachium flectit.

Extensores antibrachii, flexoribus longe fortiores, maximam massac muscularis brachii partem sistunt.

Ex quinque revera componuntur capitibus, fere omnino, etiam in insertionc, distinctis.

Hormu duo inferiores et posteriores extremo superiore per lineae circiter spatium uniti, a hrachii facie extensoria, hic anteriore, orti, ad olecrani partem inferiorem ejusque basin tendunt. Alter alterum ita tegit, ut ille nonnisi summac facici auteriori, hic dimidio ipsius supcriori nuiatur.

Terlius, ante hos positus, a scapulac margine inferiore, statim ante carunn glenoideum orihur et, primo in extremo inferiore nonuilul tectus, medio olecrano inseritur.

Quartus, hunc statim exripiens, ex eodem margine medio oritur, ipsum tegit, et cum primo in fine infimo nonnilil confluit.

Quintus, ipsi vicinissimus, a scapulae margine inferiore ortus, apici olecrani inscritur.

Ounnes lii musculi revera alb se invicem separati sunt, ut fibrac nullae discerpantur, si singulos ob oculos ponere relis.

Fortiter antibrachiom sursum trahunt alque extendunt.

Massa muscularis antibrachii rotundit, brevis sed crassissima et fortissima.

In facic externa s. anteriore, extensoria, primo altissimo loco ex margine ossis brachii cxterno oritur extensor radialis, satis crassus, tendine in carpo in tres fasciculos fisso ossibus metacarpi secundo, tertio et quarto inscrlus.

Iufra hunc, all codem tectum magis introrstum, alinm invenio, longe minorem, ex codem fere loco ossis lunneri longe radinm ad ossis secmudi primi ordinis Carpi dorsum tendit. Sine dubio supinator longus est, ad carpum usque extensus, posilu omnino cum loc musculo coureniens.

Sequilur tertius, a condylo externo ossis brachiii orlıs, profundus, radii faciei externac dimidio superiori insertus, supinat or brevis, valde temis.

Tegitur hic extrorsum extensore digitormm communi longe forliore, digitis tertio, quarto et quinto tendinibus prospiciente.

Sequitur lune latus et crassus, uhnaris externus, phalangi primac digiti quinti insertus.

Praeterea tres adsunt minores et profindiores.

Ex his abductor pollicis a dimidio snperiore ulnac ad pollicis ossis metacarpi basin tendit.

Magis extrorsmm exteusor pollicis et digiti indicis ab olecrano ad phalingem ungnicularem utriusque digiti abit.

Ultimus, temissimus, inter ulnarem externum et cxtensorem communem a condylo externo ad digiti quinti dorsum abit, ad phatangem uspue tertian extensus.

In facic flexoria, primo Pronator teres, longus, sed tenuis, parum obliquns, a condyli interni extremo ad medium radiun decurrit, quem cum uhna fortiter flectil.

Hunc sequuntur omnium in antibrachii sitorum crassissini, massam rolundinsculam sistentes, singuli simul compressi a margine radiali ad uluarem.

Primo radialis internus a condylo interno et dimidio interno extremi inferioris faciei humeri flexoriae oritur, primo ossi in margine radiali carpi positos hiuc pro sesamoideo habendo, dein ossi metacarpi secundo insertus.

Flexor digitorum communis, maxime in directione modo dicta compressus, sed a centro artus versus superficicm latissimus, ut illic vix lineam cum dimidia, lic fere pollicem aequet, a con- 
dylo interno brachii et uhnae facie postica fere tota orlus, tendine crassissimo, mujus fini inferiori duo ossicula ad Sauriorum Dasypodnmque modum insunt, ad manum tendit. Mlic in metacarpo quinqueliurian dividiunr. Singuli tendiues platangibus unguicularibus digitorum inseruntur.

Loco inter ortun teudinis communis ipsinsque diraricationem fere intermedio faciei inferiori, pahuari quatuor iusident musculi parvi, crassiusculi, quorum extermus maximus, sccundus, digito indici respondens, minimus est. Ili tendinibus longis, tenuibns, nulli ossi sed membranae interdigitali intexunur versus manus extremum, eamque et flectere et plicare possunt.

Praeterea musculus flexor communis neque longns, neque brevis adest ullus. Defectus, ni fallor, et musculi et tendinum summa crassitudine large compensatur.

Tertius horum musculorum, crassitudine et compressione cxcedentium, ulnaris internus, duobus capitibus omnino distinctis, brevi a condylo brachii interno, fongo ab olecrano uhace ortus, primo ossi pisiformi, dein ossis metacarpi quarli et quinti basi inseritur, hic cum interosseo externo s. abductore digiti quinti confluens.

Manus musculi breves nomnisi in vola conspiciuntur.

Interosseos refermut, ita dispositos, ut singulis ossibus metacarpalihus insideat musculns brevis, crassinsculus, versus extremum anterins bilidus et tendinihus duobus, lateralibus phalangi primae ejusden digiti, a cujus osse metacarpali oritur, insertus. In quinto nonnisi umus, extermus adest.

Neque pollex neque digitns minimus mnsenlis propriis gandent, ut hic pulcherrime confirmetur sententia, secundum quam musculi proprii utriusque digiti nomnisi pro musculis interosscis magis erolutis et in plures rentres divisis habentur.

\section{ศ. 19}

In extremitate posteriore Glutaeus maximus, musculus amplissimus, fortis, triangularis, lato ortn ex supremo osse ilim, spinis sacralibus et caudalibus primis progrediens, pone et infra femur et crus ad pedem tendit, versus cruris finem in tendinem fortem, brerem transit, quo in plantae eutem abit, simnl ossis, aculenm sustentantis facici cxtcrnac inseritur, quod introrsum et antrorsmm trahit.

lufra hunc ponitn musculus longe minor, triangnlaris, transversns, e spinis caudalibus dnabus primis ortus ad medium femme, teudiue infra trochanterem et mediun totins ossis externum fine recto inserendus. Femmextrorsum fortiter rotat. Hunc pro gluta e o un edio habeo.

Gluta eus tertius ante hunc, in finc inferiore nomihil ab eo tectus, duplo major, crassior, oblongus, a facic ossis ilium externa ad trochanterem externum descendit, cui, statim ante glntaeum secundun positus, inseritur. Femur extrorsum rotat et extendit.

Ex rotatoribus obturatoriun externum invenio maximum, quadratum magmum quidem, sed hoc multo minorem et tertimn, minorem, quem pyriformem dixerin.

Obturatorius externts ex maxima foraninis obturatorii parte ad faciei ossis femoris posterioris partem superiorem tendit, hic plagae trochanteribus intermediac insertus. Sneto majoris quadrupedum numeri more femm potius retrorsum trahere, quan extrorsum volvere potest.

O uadratus, infra ipsum, ab osse ischii provenit et faciei posteriori trochanteris externi inseritur, codem fere modo agens.

Pyriformis, tenuior, longus, transversus magis retrorsum ab spina processus transversi vertebrae candalis terliae ad femm tendit, cnjus margini externo infra glutaeum secundnm tendine tenui inscritur. Hic fere omnino sueto more femmr extrorsum rotat.

Reliquos in musculos caudales inferiores mutatos crediderim.

Ex oppositis lliacus interuus, tentus, brevis et latıs, fere omnino extra abdomen positus, maximus, rhomboidens, basi trochanteris femoris interni et lineac internac asperae ab eodem prodeunti ita inseritur, ut fere totam femoris longitudinem meliatur.

Fortiler praccipue femur introrsum rotat.

Psoas maguus et iliacus internus solito etiam magis in mum confluxere nusculum. Ille a vertebris lumbaribus et ossis sacri parte superiore, hic a facie ossis ilimn interna oritur. Uterque latissimo tendine trochanteri interno aflixi, temur valide flectunt.

Liceat hic adjicere et P'soac minoris descriptionem, quamvis hand ad femm ipsum pertingat. Hic modo descriptis longe major, immo ad maximos Oruithorhynchi musculos pertinens, a quinque vertebris dorsalibus ortus, tendine fortissimo eminentiae ileo-pectineac, ipsius gratia tantopere erolutae affigitur. 
Valide pelvim flectere et sic et artus inferiores et candam rotationis ergo deprimere polest.

Inter musculos femorales e flexoribus Biceps, solito more et uno capite a tubere ischiadico prodit, et temuissimus latissimnsque versus inferiora latescens supremae fibulae et aponemrosi crurali inseritur. Qumm transversus decurrit, rix crus flectil, sed fortiler extrorsunn rolat.

Sartorius, musculus crassus, fortis, fere rectus, marginem faciei internum femoris superiorem tegit. Tibiae extremo superiori ita a parte interna inseritur, ut crus introrsum rertat.

Ipsmm sequitur Gracilis, latissimus, el salis crassus, totam faciem internam femoris tegens, a symplyysi ossinm pubis et tolo osse marsupiali orlus, lato extremo inferiore tibiae margini superiori panllulum supra ipsius medium insertus. Crus fortiter introrsum rotal.

Semitendinosus et Semimembranosus hoc crassiores el camosissimi, sed minus lati, sucto more tibiae supremae, neuter longo tendine, neque superiore, neque iuferiore gaudentes, insermutur.

Inferiori accedit musculns a superiormm rertebrarum caudalimn processibus trausversis ortus, cum ipso in insertionis in tibiam loco confluens, a glutaeo externo tectus, el nonnihil cum ipso unitus, ut forsan pro ipsins parte haberi possit.

Extensores nomisi duos inrenire potui, rectum, s. superficialem el cruralem, s. profundun simnl vastos referentes. Rectus hoc duplo longior, crassitics eadem.

Adductores practer pectinaenm adsunt duo, in femina longe melins quam in mare divisi.

P'ectinaens, minimus, tennis, longinsculus ab cminentiae ileo-pectincae basi, ad medium marginem ossis femoris inlermun tendit.

Adductor primus, relicuis temuior, presse pectinacum sequens a rami horizontalis ossis pubis parte externa ad condy lum intermum ossis femoris; secundus, longior et crassior, a reliquo ramo horizontali el symphysi itidem ad condyhum intermum, magis introrsum et retrorsum tendit.

In cruris latere extensorio sequentes pounntur musculi.

'Tibialis anticus capilibus duobus, satis distinctis, externo, lougiore a facie patellae externa, interno breviore al) extremo snperiore faciei tibiae externae ortus, ante tibian decurrens, ossi scaphoideo inseritur. Peden introrsum flectit.

Extensor pollicis longus, a parte fibulae suprema ortus, phalangi pollicis unguiculari inserilur. Tibialem paullo magnitudine sıperal.

Extensor digitorum communis longus, major musculus longe minor et inferiore loco, mox infra basin capilis fibula superiore, oritur. Statim in duos rentres fiudiune, quormn interni tendo nonnisi digito secundo, externi huic et reliquis praeler pollicem infigitur.

Magis extrorsum, ct a sequentibus tectus, a summa facie exlerna fibulac, sed infra caput, oritur musculus minor, simplex, mox in tendinem fongun ef tenuem abiens. llic in tarso subito dilatatus, mox in quinque romos finditur, phalangibus onminn digitorum primis insertis. T'Tondines lumjus musculi tendinibus antecedentis subjacent.

Hic musculus sine dubio pro extensore digitorum brevi habendus est, qumm 1) practer ipsum nullus adsil; 2) codem modo sese labeat. Nemorabile hoc respectu, tendinem hujus nusculi per spatimn tam longum sine ulla divisione decurrere, et in pedis dorso tantopere explanari, quo musculi extensoris brevis imago quodammodo redditur.

Peron aci adsunt dno, singnli ex smmmo fibulac capite orimndi.

Superficialis, hand longior, sed panllo crassior, tendine longo inter calcanenm ad os melatarsi quintum in plantam descendit, hic in plures appendices fissns, quibus ossibus cuneiformibus, praccipue primo, maximo inserilu.

Profundus, paullo Lrevior et tennior, ossis metaliursi quinti basin adit.

Lateris flexorii musculi, nt semper, quamvis minore ac in antibrachio ratione, oppositos magnitndine rincint.

Structura liace est.

Gastrocnemius duobus capilibus oritur, altero, externo, ter vel quater majore, praceipue latione, a facie posteriore capilis fibnlae, minime antem femoris; altero, interno, a facici fomoris postcrioris parte interna, pone condylum intermum. Tendine brevi, crasso inseritur tuberi calcanei, hic in basi prominente.

Hoc musculo Solens tegitmo, ex eadem altitudine a capite fibulac, dein a maxima libiac parte orinndus. Omnino separatus est a Gastrocuemiis, et rersus narginem pedis intermun tendine crasso et brevi inseritnr tubcri astragali, illi calcanei simili.

Longe fibnlae faciem posteriorem et externam fere totam oritur flexor digitorum crassissimus. 
Tendo ipsius, cum tendine gastrocnemii decussatus, in plantam abit, subito hic in quinque ramos divisus, singulorum digitorum phalangibus unguicularibus inserendis. Ex facie inferiore tendinis communis, hic maxime dilatati, duo oriuntur musculi, digito secundo et tertio destinali. In fure antico fissi, phalangi primae insermutur. Sunt igitur musculi illi et lumbricales, et flexores perforati, superficiales.

Tendini dilatato accedit caro crassa, brevis ex planta pedis.

In facie interua decurril Tibialis posterior, a fihulac capite versus latus internum orimendns. Relicpuis longe minor tendine temui, ossiculum sesamoideun satis magnum continente hallucis phalangi primae inseritur.

In dorso pedis musculi adsunt nulli, exlensor brevis modo jam dicto in crus rumu adscenderit.

In planta pedis 1) ossibus melacarpi digitorun onnibus supponuntur musculi, iis simillini, quos in pahua descripsi, sed tenniores et magis teretes.

Practerea, quod mirmm ridetur, sed optine com lalitudine membranae interdigitalis inter utrmnque digitum extensae congruil, non hallux, sed digilus fuarlus et quintus musculis, ul primo saltem intuilu videlur, propriis, longe majoribus el magis superficialibus gaudent.

Horum primus, a tarsi margine fibulari oblique ad basin phalangis primae digili quarti descendit, quem, versus faciem fibularem phtalangis insertus, extrorsum trahit, simul flectit.

Alter, etian a tarso, sed magis ad latus tibiale orlus, infra hune musculum inter ipsum atque os metacarpi quartum et quintun decurrens, phalangis primac digiti quinti baseos lateri tibiali insertus, hune ad quartum fortiler trahit.

Ab codem cum primo osse nascilur musculus, reliquis longe crassior, longns, ossi metatarsali quin10, simul baseos plalangis primac lateri fibulari insertus, abductor digiti quinti.

Nolandum tamen, primum el tertium liorum musculorum non proprios, sed nonnisi lumbricales quarli quintique digiti esse, reliquis longe majores el a tendine flexoris ad os, sine dubio nt melins atque separatim in digitos, quos adeunt, agerent, depulsos. Hinc in descriptione flexoris communis non-. nisi dnos lumbricales, digilo secundo et terlio prespicientes, descripsi.

Lx hisce musculis communes etiam in clorso pedis ex parte conspicinutm. 
1. 20.

Quac de systemate circulationis nota sunt, ad sequentia redemnt.

Ilomius cor secundum lineam thoracis medianam, apice sterno obrerso poni, pericardio forli inchudi et duobus atriis totidemqne rentrienlis, septo omnino perfecto, nt neque inter atria neque inter ventriculos detm communicatio, componi tradit. Addit, renam caram superiorem adesse duplicem, quamum sinistra renam sine pari accipial, aortam reliquasque arlerias esse angustas ${ }^{1}$ ).

Curicrus primo foraminis oralis obliterationem completan el cordis fabrican cuadrilocularem confirmat, dein cor conicum, atrium dextrmm magnum, sinistro tonge tentuius, ilidem ventriculum dextrum valde tenuem esse adjicit. Diligenter praceipne valnulan renosam utramente describil, dextran longe magis musculosam, quan membranaceam, tribus fasciculis carncis conpositan, sibistram nonuisi membranaceam, margine libero musculis papillaribus tribus affixitm aflirmans ${ }^{2}$ ).

IIomius Echiduac Mystricis cor omnino cun Ornilhorlynchi conrenire, nec rasa sangnifera nisi venae cavae superioris simplicitate ab lujns animalis rasis differe memorat ${ }^{3}$ ).

Onac rgo inveni, liaec sullt.

Cor recta ponitur et forma inter rohundısculam el oblongan media gandet.

Pondus ipsins drachmae 1 uniss cum dimidia, ratio ad tolum jgitur corpus uli 1 : 170 est. Pericardio includitur firmo, fibroso - scroso, diaphragma minime tangente, nec cum corde alio, quam reflexionis rersus ipsins fariem externam loco romuexo.

Atrimm dextrum maximum, sinistro longe majus est. Divisio inter simmn atque auriculam in nentro adest ralde distincta, qunm antriculac obtusae, breves el altae sint.

Recipit venas principes, renam caram snperiopen dextrom ju ipso fine supremo; sinistram in facie inferiore; inferiorem, simpliecm in eadem, sed magis dextrorsum. Omnibus orificiis ralvulae adsunt, renac carae inferiori et superiori sinistrae singulis tantum una, maxima, dextrae superiori duac, minores, externa major, interna mintor.

In septo rersus extremm superius foream quidem inveni valde profundam, sed minime perviam, ut igitur el hie, ul in Ilomiano el Guvieriano foramen ovale clausum frucrit. Riem in ulloque rorle sic se habere vidi. Vena coronaria cordis maxima fossam lianc, dextrorsum a vena cara inferiore, intrat.

Ventriculus dexter, parietibns temussimis formatns, sintistro triplo latior est. Facies interna parmm inacequalis est, adsunt tamen apiecm versus musculi papillares, et trabeculac. Basis in conmm aculissimnm, arteriarum puhmonalem qui edit, producilur. Ostimn arteriosum tres continel valvulas.

Ostium renosum, fere rolundum, valunla clanditur simplici, scmilunari. Curierius eam nomnisi concaro ventriculi parieli respondere dicens, minus perspicue loqui videtur, qunn uterpue, et anterior s. dexter, et posterior, s. sinister, a septo formatus, convexi sint. Illi revera obreptilur et insidel margine lixo. Recte Cunierio maxima ex parte carnea dicilur, qumn nomisi pars libera, anterior, margine leviter concaro circunscripta, membranacea sit. Fasciculi in unssculares, ad ipsam el e septo el e paricte antico tendentes, ad tres ordines reduci possunt. Inferior, major, e pluribus fasciculis compositus, ex septo medio, infero ad extremum rabulac inferius abil; anterior ex parietis anterioris parte inferiore recta ad basin valuulae adscendit, ubi cum superiore, ex summitate septi descendente confluii.

Fabrica sic nomnihil magis composita, qnam Cuvierius descripsit, appared, allamen sinilitudo quacdam enm arium valnula venosa dextra et propter camositatem el propter figmam minime praetervidenclia adest.

1) Phil. Tr. 1802. p. 74 .

2) Aunt. comp. T'. V. p. 293.294.

3) Ibid. p. 352 . 
Ventriculus sinister, anguslissimus, sed parielibns crassissimis composilus est. Valvula renosa, ut recte Cutierins monet, ommino membranacea est. Practerea ideo quocue atd ratrulartum mitralium tymun formata est, yuod totum ostium circmndal. Laciuia anterior, superior, longe maxima, musculis duobus, fortissimis, ipsins extremis amexis continuatur, opposita tribus, multo minoribus per tolum marginem milur.

Atrium sinistrum longe carnosins, sed angustius sinistro venas pulmonales, dextrum duas, median, e lobo pulmonis dextri inferiore provenientem adscendeuten, siuistran e pulmone sinistro accipit.

Vasa omnia, praccipue veuas, valde lemes offendi, arterias pratecipue angnstissimas, ul praecipue muscnlos, in extremitate posteriore el glindulae veneniferae ductun perscrutans, hic minns accuratam sistere quean descriplionem. Fenninae artus posteriores ietu sclepetario dilaceratae erant.

Aorta areun format, e cujus cavilate, laud proximi, mini tres secedunt, truncus anonymus dexter, carolis sinistra el subclavia sintistra, 111 hor exemplo, ulriusque auinalis perserntatione confirmato, corum animalium augeatur numerus, jam secundun exempla alia, liu nota, longe major illo, quem Curierius exhibuil, quihus codem ac in homine modo rani principes ex aorlae arcu abeunt. Vix unquan tam longe iuler se distantes inreni, quum in parro animali singulos fere duac lineae iutercedaut.

Truncns inuomiuatus, emenso trinm linearum spatio, in subclavian, fere duplo majorem, et carotidem commmem diriditur, in laryugis regione in cerebralem et fucialem sueto more abenutem.

Subclaviae ex oaro thoracis exitum jam supra dixi $\left.{ }^{1}\right)$. Emenso laud unius lineae spatio ramm, superiorem, satis forlem, cerricales al scapulares edeutem, inferiorem, ejusdem magniludinis, rertebralem, miltit, el sex ad septem lineas progressa in axilla in duos findilur ramos, quorun major, retrorsun tendens, in pectorali majore et nusculo culaneo absumilur, minor in brachium abit. Jlic in medio circiter brachio arteriam radialem minimam enillit, fere tolus cum nerro mediano per foramen ossis Immeri ad autibrachium proficiscitur.

Aorta thoracica el abdominalis nilhil iusoliti offermul. Cocliaca et mesaraica superior separalae ex aortae trunco prodemu. Illa sueto more mox in hepaticau, splenican el corouarian reutriculi dividitur. Splenica ad extremum splenis superins in dnos secedil ramos, singulis lobis prospicientes. Arteriae renales simplices.

Initio pelvis aorta, in iliacas el caudalem, ipsins continualionem, dividitme.

Extremitatis inferioris arteriae praccipue ex lypogastrica, iliaca extema majore, proficiscmutur, ob flexornm cruris magnum incrementum. Celerum iliaca externa sueto more decmrit.

Venac carae, $n \mathrm{t}$ jam in cordis descriptione monumus, adsunt tres. Harmm utraque superior venis subclaviis, jugularibus internis, valde parris, externis, maximis, internas magnitudine longe vincentibus efficilur. Subclariac et jugnlares externae maxime intunescunt propter magnitudiucm venarum musculormu colli et abdominis, praecipue musculi cutanei, quae regione axillae maxime dilatatae direrticulum quodammodo fornant. Vena cara superior ulraque ceterun pone appendicis sternalis ramum transrersum rase magno transverso minntur', sine dubio pro parte transversa dextra renae snbclaviae sinistrac co'um animalium habenda, quibus nna tantmm adest rena cara superior.

Cara dextra solito loco, sinistra codem ac in magno animalimm numero ita atrimm dextrum intrat, nt totum sulci circularis dimidium inferius percurral el in faciem inferiorem aperiatur.

Cavan superiorem sinistram, ut recte jan Ilomius monnil, intrat azyga. Usque ad medimm circiter thoracem adsunt duac, dextra el sinistra, quarum haec aortac lateri sinistro presse apposita est, in thorace medio dextra columnam verlebrarum transscendil el sinistrae initur. IIaec ad pulmonis sinistri extremum fere superins usque adscendit, tme antrorsmm et deorsum flexa, arteriam pulnonalem transscendens, carae sni lateris inseritur.

Cava inferior, simplex, a dextris posita, atrio juxla superiorem sinistram inserilur, postquam sueto more pollicis et quod excurrit fere spatimm in thorace a diaphragmate ad cor percurrit. In liepate fere ad phocarum lutrarimque modum valde dilatatur, supra el infra hoc subilo angustior.

Fornatur solito modo renis renalibus simplicibus lumbalibus el artumm inferiormm truncis.

Venam portarum salis anplaun el cavâ crassiorem inveni. 


\section{21.}

Systema nerrortm, execplo nerro diviso, fere omnino non exanini fuit suhjectum. Cl. Itomins in diligenti quoad viscera descriptione, se encephaton in statu mintune ad dissectionem apto invenisse, disertis rerbis dicit, necpue relicpuis seriptoribus contigit esse felicioribus.

Hine maxime lactor, me in specintule me masenlo gravissimam hane partem in stalu catchus saltem bono inrenisse, ul, quamris fribibilus et facile frangendum, tamen eximi et satis pervestigari poluerit.

Cerebrum, cranii carum exacte implens, circumdahatur primo membrana fibrosa, satis forli, iu regione processus falciformis ossei nomisi in sulcum deperessa, minime rum ipso connexa, inter cerehrum atque rerchellum tentoriun minime osseum formante. Quae sequitur, membrana vasculosit, itiden crassa, laxe circumdabat rerebrum, vix gy ris sulcisque execplo in eerebello, notatum.

Poudus tolius massac cerebralis duas drachmas aecunabat, ratio igilur ad totum corpus uti $1: 190$ cral.

Cercbrum drachmam nuam com dimidia, ccrebcllum cum medulla oblongata triginta grana pendebal; ralio igitur illius ad hane partem uli 1 : 3 erat.

Cerebrum, nli dixi, fere onnino lacte, nonnisi in facie superiore versus marginem utrinsque Jemisphacrii interunn, sulcis quibusdimn vix perspientis, forsan nomnisi a spiritu vini productis, prateditum est, oblougunn, introrsum acuminatum.

Pollicem unum longum, in extremo postico, ulbi latissimum, pollicen cum duabus lincis, iu cxtremo antico, uhi lalissimum, quatuor lineas latum, et quincue lineas alunn est.

Corpus callosum adest quidem, sed breve, ynum land quatuor lineas longitudine acquet. Memorablhilins ctiam videlur, in dimidia dno lateralia, linea mediana haud confluentia, esse disjunctum. Equidem saltem in fariebus sese spectantibns internis mullun dilacerationis restiginum inrenire potui.

Ventriculum lateralem obtulit angushum propter parietis externi smmman crissiludinem, temuibus superiori el interno,

Corpns striatum longissinum et angushum quidem, sed satis magnum.

Thatauns opticus minimus, cum opposito confluens.

Fentriculus terlins angustus. Commissura anterior maxima.

Eminentia quadrigemiua magna, posterior tamen vix pereipienda, ut fere higemina esset.

Cerebellum, salis magunu, latum el altum, in medio altissimum, apperndicibus duabus, parris, in forea rranii, uti plurimis animalibus conrenit, condili, includitur. Pars nuedia, tonge maxima, nudecim suleis transtersis redditur inaequalis. Hlic antem nonuisi sex lobis, quibus arbor vilae basin dal, componitur, anterioribus simplicioribus, posterioribus magis complicatis majoribusque.

Mcdulla oblongata satis magna, el, uhi cranium relinquil, quatnor lineas lata, in facic inferiore fabrican satis memoralilem exluibet.

Juxta lincam medianan eminentia gandet angusta, sursum angustata, in crus cerebri abeunte, cminculia igitur pyramidali.

Hanc antrorsum sequitur alia, longe latior el altior, antrorsum valdopere dilatata, triangularis. Eminentiace olivaris sedem tenet, et magnae nervi trigemini erolutioni respondere videtur. Hae partes libris longitudinalibus formantur.

Secquitur stratum fibrarun transversarum, nodus cucephali, satis tenuis.

Ex hoc emergit emincutia magna, crassa, rolundusculiı, lacris, directione el situ emtinentiae secmulae, externae respondens. In uic insidet nervus dirisus, minime antem continualis fibris, nam in utroque latere codem exacte modo, sine ulla fibrarum dilaceratione utranque partem nonnisi menubraua vasculosa junctam solvere polui. Iloc in utroque excmplari obscrvavi.

Sulcus nnedianus in facie inferiore adest profindissimus, praccipue in parte anteriore valde de- 
pressus, ut hic fere omnino in dno dimidia Iateralia secedat. Hnic sulco respondet eminenlia longitudinalis, in descriplione cranii dicta.

Ex neris nomisi dirisum, opticum et olfactorium satis bene perspicere pohi.

Divisus, ounium maximus, in ortu modo descriptus, lineas quinque latus, muam alıus et crassus, antrorstum tendebat.

Nerrns oplicus, secundum oculi dimensiones, valde parrus. Uterque, in linea media coïens, chiasma oblougum format.

Nerrus olfactorius, magnus, sed diriso longe minor, statim pone eminentiam quadrigeminam a facie hemisphacrii cerebri inferiore ortus, circa crus cerebri flexus, facie superiore hemisphacrii faciei iuferiori annexus, duch salis recto antrorsum ferebatur. Carilatem equidem luand deprehendere polui.

Medullam spinalem hand bene inspicere polui, quac ad ipsins neros pertinent, haec sunt.

Nerrus cervicalis primus, valde parvus, in sulco facici condyli anterioris insculpto ad foraminulum in processu transverso supremo inveniendum decurrit, quo penetrato sueto more dividitur.

Cervicalis secundus inter allantem el epishophacum exit, mox cum sexpente unitus. Foramen in epistroplaco $110 n$ ad neros, sed ad rasa spectare, persnasım mihi fuit.

Ilic el cervicalis tertius, quo ipse nomihil minor est, e maximis cervicis, praccedente el duobns sequentibus longe major est.

Nervi extremitatis anterioris e sex paribus, quinque cerricalibns inferiocibus, primo dorsali oriuntur. Ex lis quatuor inferiores, fere aequales, duobus superioribus longe crassiores sunt. Unimulur ila, nt terni in plexum coëant, quorum inferior superiore crassior el longior est.

Inferior in ramos tres diridinu, quornm suprenus relicquis longe numor est.

Ex his inferior fere tohs in musculi culanei parte media absumitur; medius eidem dat ramos anteriores, et nervum ulnarem emiltil.

E plexus superioris parte inferiore abit nerns medians, per foramen ossis lumeri praceipue ad flexores manns tendens, dein truncus nervi radialis et numsculocntanei communis, longe crassior.

Nervi dorsales, satis magni, id peculiare habent, quod non inler hinas rertebras, sed, foramine, quo arens singularum vertebrarum basis pertunditur, per vertebram unam tántum canali medullac spinalis exemnt.

Plexus humbo-sacralis quinque nervis formatur, dnobus dorsalibus posterioribus, duobus lumbalibus, mo sacrali. Supremus reliquis, inter se aequalibus, dimidio minor est. Plexus ipse in dinidiun superius, e nervis duobus supremis cum dimidio tertii, et inferins, codem modo e tertio, quarto et yuinto compositmm secedit.

Ex illo oritu prino crmalis, mox in ramm culaneum ad pedem usque decurentem et muscularem divisus, dein, sed ex moctue tantum dorsali, obluratorins.

llic nervum ischiadicun crassissinum producil, prinscuam pelvim linquit, in peronacum et tibiaIem divisun. Ille in medio femore in muscularen el cntancun secedit, hic, ranis ad flexores cruris datis, sneto more in erure et pede dividitur.

Nervos candales octo, inter anteriores candac rertebras norem sneto more suptra processus transversos prodenntes mumerari, sensin longiores et exiljores. Vix egressi finduntur iu ramum superioren, exiliorem, inferiorem, crassiorem, quormm hic in dorso candac, ille, inter processus transversos penetrans, in facic inferiore, inter musculos candac, ipsis prospiciens fere rnsque ad apicem decurrit. Ramorum confluxu in utraque facic plexns formatur longitudinalis, prilclucrimus.

Nerns pnemmogastricus, satis fortis, a sympathico maximo omnino separatus, initio arcle cum hypoglosso unitus, praeter laryngeum in collo 1 millum rammo edit. In summo thorace 1) recurrentem, sucto more in dextro latere circa anonymam, in sinistro circa aortae ortum reflexum el inter cartilaginem thyroideam et cricoideam laryngem intrantem; 2) nerros cardiacos, inter aortam et caram superiorem cor ademules; 3) plexmm pulmonalem et 4) ramos ocsophogeos atque gastricos edit.

Spinalis accessorius, pneumogastrico crassior, ad mulatorem capitis, praccipue antem cucullarem tendit.

Facialis, satis parvus, post meatum auditoritum emergens, circa ipsum antrorsum flectitur et fere totus in risorio absumitur.

Hypoglossus, puenmogastricum fere aequans, primo descendentem anastomoticnm ad nerrum cervicalem tertium millit, dein antrorsum flexus, inter mylohyoideum et genioglossum sueto more ad musculos linguales tendit. 
Nerrus trigeminus sine dubio omninm in Ornithorhyncho memorabilissimus est. Cl. Blumenbachius jandudum ingeniose in ramos, ob quorum erohntionem insignem praecipne attentionem meretur, infuisirit et icone illustravil ${ }^{1}$ ), ni autem graviter fallor, ols exsiccationem animalis mirabilem structuram minus perfecte exhibere potuit.

Rationem hujus nervi ad reliquos e cerebro exenntes jam supra²) diximus, hic, enm reliquos ounce, nou tautum cerebrales, sed totum systema nervosum periphericun simul fere aequare, adjicimus. Ifoc ex collatione diametrorum facile patebit.

Nerri trigemini radix simplex, modo jam supra dicto, lineas quincpue lata, mann crassa est.

Nerrus opticus vix tertiam lineac partem, facialis et acnsticus dimidiam, relicpui omnes simnt sumti nuam enu dimidia in diametro metimutur. Spinalibus, simul sumtis, si tres lineas tribuo, nimis largior.

E cerebro egredicus, arcum format, concavitate processui cerebri supra dicto obrersmm. Statim, rix dimidiae lineac cmensus spatium in ramos tres ila diriditur, ut primus nounisi cursus secundi vidcaltur.

Ramus primus, omminm longe minimus, post lineac circiter decursum in duos fere aequales diriditur. Tlorum superior, ethunvidalis, louge maximus, nasum intral, nerro olfactorio suppositus, et primo surenlo post septem circiler linearum decnrsum per foranen, in descriptione faciei ossinm dietum egredilur, ad summae faciei cutem tendens, dein altero rano, longe majore, inter extremum anticum ossis nasi et os intermasillare egrediens, extremo cultis facici anteriori el naribus prospicil. Juferior, longe minor, cum optico e cranio egressus sucto more in orbita el, cì cagressus in fronlis cule cl mnsculi cutanci extremo antico distribuitur.

Ramus secuudus el tertius praceipue ob magnitudinem et subtilitatem nerrornm labialium ex ipsis prorenientium, altentione digni sunt.

lianns secundus, omninun maximus, crassissimus, duas lincas latus, unam cum dinidia crassus, fer foranen rolundum rranio egressus, praceipne in infraorbitalem abit. Ilic, oculi bulbo supposilus, mullo septo osseo ab jpso dirisus, parietem anteriorem orbilac perforal, sic canalem intrans infira-, rerlius anteorbitidem.

Hoc egressus in ramos chuos discedit, superiorem el inferiorem, lamina carlilaginea supra dicta divisos, naso et labio superiori prospieicules. Superior nasales, inferior labiales superiores refert. Vtereque maximus in naso el labii superioris parte laterali tota ita distribuitur, ut parieli superiori, praceripuc antem margini laterali fariei respondeat. Lhi foramine infraorbitali exeunl, simul duas lineas lati, fere minam alti, spatio, duos pollieces longitudine, quatuor lineas latiludine exredenti inveminntur. Filia nervea uliene intertexnntur, anastomoses formant el rersus marginem rostri sulthilissime divisa fininutur.

Ramns palatims, longe minor, duobus ranis, posteriore, minore, per formencu palitinum posterius, anteriore, majore, per anterins egresso, membranae palati prospicil. Hic praecipme ad Jacobsonii organou teudil.

Thanus terlins, secundo nomilhil minor, latior quidem sed longe tenuior, compressus, per foramen ovale subito rranio egressus, solito more magna ex parte musculis manducatoriis, praecipue autem labio inferiori prospicit.

Autequam maxillac inferioris canalem intrat, in duos dividitur ramos. Iorum posterior, longo minor, inferior, suyerficialis maxillac inferioris maroinem inferiorem tegens, mylohyoideo surculum dat, et demo bifarian dirisus, fere tolus in labii inferioris parte media et posteriore alssumilur.

Anterior, ramus fere totus, primo ramulun haud crassnun lingualem edit. Ilic mylohyoideo surcnlum anteriorem dat, dein, trajectis fibris hujus musculi, inter ipsum, genioglossum el hyoglossum, nullis ramis emissis, ad liuguac membranam tendit. Dein canalem maxillarem intrat, et in nerrum maxillarem inferiorem crassissimumn mulatur. Pars ipsins nonnihil minor, posterior foramine magno, in facie externa maxillae ante dentes molares posito, exit et in labinm inferins penetral. Vix egressus in rammu superiorem, majorem, inferiorem, minorem dividitur. Ille ad partem labii inferioris superiorem, buccalem, lic inferiorem, cutancan tendit. Reliqua pars nervi, earne major, emenso tolo canali maxillari, per foramen mentale exit, labii extremo antico prospiciens.

Nervus intercostalis maximus hand valde erolntus, truncus tamen, fuem dicunt, per totum collum a pneumogastrico, crassiore, separatus decurril.

1) Vergl. Anat. Taf. 1.

2) Pag. 34 . 
ก. 22.

Priusquam apparaluum descriptionem aggrediar, cara thoracis et ablominis describenda sunt. Ex his carum abdominis amplissinnm obserrari, longiludine a symplysi ossinm pubis ad sterui apicen atque diaphragmatis centrum tendincum quincque pollices, summa latitudine circiter in media lougitudine pollices tres cun quatuor lineis moliente. Praceipne iu dimidio supremo in latitudinem expanditur, in parte inferiore, praccipue quatemus a petvi formatur, valde contrahitur.

Summum locum tenet liepar, satis magnum et ita per totan aldominis latitndinem transserso positum, ul, choad hane dianetrum iden in sinistro ac dextro latere ocempet spatium, in dextro nonnihil tantum altins sit. Ligannento suspensorio falciformi a media ipsins facie superiore exacte in linea mediana ad centri tendinei diaphragmatis medium tendente, priteterea in dextro latere duobus, in sinistro tribus ligamentis similibus, satis longis, ilidem a loborum lıpatis facie posteriore ad centrum tendinerum abeuntibus, ut igitur cl. Blaimillius miuns recte talium ligamentorum defectum sit suspicatus ${ }^{1}$ ), lunic musculo afligitur.

Pone lepar el nomuihil rersus latus sinistrum oesophagus in reutriculum abit.

Hlic recta fere infra liepar retrorsum descendit. Li ipsins extremo superiore a dextris dhodenum prodit, suhito descendens, tunc post breve spatium dextrorsum pergens.

Tolus intestinorum tractus a rentriculo ad anum usque mescnterio satis laxo el amplo parietibus abdomiualibus affigitur.

Oritur mesenterium a facie columnae rerlebralis inferiore ab liepate usqne ad pelvis finem fere mediâ, nonnisi versus inferiora nounilıil ad latus sinistrum vergens et in duo dimidia divisun dici potest. Hortun dextrum anterins, longe maximum, quamris initio angustius in summam amplitudinem crolutum, totum tenue et maximam crassi partem couliuent. Longe major teunis par's et crassi inilinu simplici duplicatura laxe contincentur, magna aulem dextra duodeni mediaque crassi pars arcle inter se comectuntur laminis mesenterii ex ipsorume regione secedentibus atque ad utrumcrie abemulibus.

Pars sinistra, scmilunaris partem crassi sinistram el rectum columnae vertebrali affigit.

Inter portam licpalis, duodeni initium et ventriculi margiuem dextrum omentum minus, salis amplume expanditur, cum pariete posteriore peritonei cartm formans, sucto more dextrorsmu foramine Winslowiano natgno cum reliquo caro abdominis communicans et pone ventriculum in carvm omenti majoris abicils.

Hoc margine superiore a duodeni initio et rentriculi margine courexo, inferiore, sinistro a liene, maximo, ortum, ab loc organo, obliche a ventriculo retrorsum el dextrorsum ila posito, ut fere totam abdominis expleat longitudinem, dextrorsum ita expanditur, nt maxime crolutum tohm iutestinorum tractum tegal, quemadmodum in fignua expressi. Margine inferiore faciei snperiori et sinistrac partis mescnterii sinistrac affigitur.

Lien, ul monui, maximus et ex cruribus duobus, dextro, longe majore, sinistro, minore, extremis superioribus quac conflunnt, compositus, non tantum ventriculo, sed parte omenti majoris inferiore crasso quoque sinistro el recto affigitur. Longe maxima omenti pars libere pendens a sinistris ex crure lienis dextro, minor tamen ex sinistro quoque progreditur.

Longe totum fere canalem intestinalem maximum glandularum conglobatarum numerum, valde propinquarum, grani milii et quod excurril, magnitudine rideo.

Tres ad quatuor tantum lineas a canali intestinali procedunt, neque, ut Momius dixil, per mescrtterium sparsae sunt. Initio jejnui el crassi majore, quam in reliquis locis, adsunt copia.

Neque in mesenterio, neque in omentis adipem inveni. Et hoc, et colon appendicibus cpiploicis destitui, recte Homius momuit.

1) L. c. p. 29. 
Intestimum temte, quod primo deorsmo, dein trausverse dextrorsum progredi dixi, lato ffexu munc primo dextrorsun expauditur, dein post spatinum sex circiter pollicum, quo colo modo supra dicto alligatur, sic emensinu, angustato mesenterio columntice reptebrali appropincquatur, subito autem dilatato denuo mesenterio in magum gy rormu numerum evolvitur. 'Tenue et crassum versus abdominis extremum posterins a dextris unila inseni, ul tamen propter sumnan litxilatem mesocoli, nuinue a mesenterio ullo modo discrepantis, locus hic niluil livi habere videalur.

Omuino in codeur cun partibus digestioni inserviculibus caro el systema uropoëticum et generatiouis ponnutur.

Reues el capsulac suprarenales maxima ex parte peritonaco tecti libere in carum ipsius prominent. Vesican urinariam in tolo ipsins ambilu peritonaci plica arele circumclatan video. A fancici ipsius anterioris linea mediana plica ampla, triangularis, pollicem alta el a pariete abdominali rersns resican ipsam lata, sic igitur pulcherrime ligannentum hepalis suspensorim intaus et septum medimu inter latns dextrum el siuistrum melins quam in unirersum revocans, ad parietis abdominis anterioris partem inferiorem exteuditur.

Testes, infra renes, ul ipsos allingant, posili, pollicem el fuod excurrit extremo iuferiore a symplysi ossinm puhis distantes, nee non epididymides el duclus delerentes mesenterio triangulari am plissimo, ab anterioribus retrorsm valde angustato, partecti peritonaci posteriori laxe adlacereut.

Carmm lhoracis, diaphragntate omnino a ravo jan diclo separalun, longe minus, imo parrum, sueto more cor el pulmoues, tunicis propriis serosis inclusos continet.

llis pracmissis, jam ad singulos apparatus ila arecelo, ut primo sensumu, dein digestionis, 1 unc respirationis rocisque, postéa uropoèseos, ullimo loco generationis organa describam.

\section{SENSUUMOR(:ANA}

$$
\text { I. } C \text { ll } l \text { i s. }
$$

\section{f. 21.}

Jam in descriplione formae externae culem quoad pilos depinxi ${ }^{1}$ ). Superest hic, ul de reliquâ

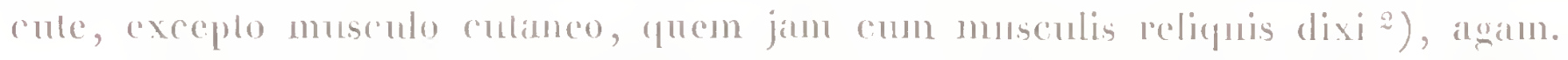

Culis ipsa lima el crassissima est, fere ubique linean aequans. Mnsculo subculaneo ubique firmissinte adliteret.

Cl. Ilomins mari, quen dissecuil, adipem subcutanem unllum, feninate ubique adfuisse, tradit 3 ). Iraue equiden hand invenio differentian, ideoque minime sexualem habeo. Adipem in ulroque animali, exceptî caudi, mullum, hic aulen maxima copia video. Simul cratssus cost, el firmus, ut longe

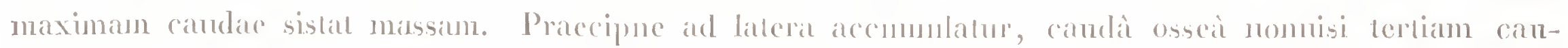
dac tolins latidudinis referente parten. Practerea in ficcie inferiore majore quan in superiore adest copia.

Cutis in facie, manibus pedibusque labitum jan supra descripsi. Jan ad reliqua orgaua transiturus, recte, ni fallor, gustus organon ad partinum digestioni iuscrviculinu ablego, lice nonnisi de ature, oculo el naso acturis.

$$
\text { II. } A \text { u } \quad r \quad i \quad s \text {. }
$$

Auris orifichum externum quoal silum, formam, ct magniludinem jam supra iudicarimus. Tendit ex hac apertura meatus carlilagineus secundum cranii lalus deorsun et nomuihil retrorsum, dein,

3) Pug. 5. 
ubi ad basin perrenit, subito multata directione introrsum alque antrorsum vertitur. Circumvolutiones lujus canalis, cl. Blaintillio dictas ${ }^{1}$ ), in neutro animali invenire poltui.

Satis mollis cst, ut parictes concidant.

Longitudo ipsius pollicem cum dimidio, diameter transrersa tres circiter lineas metitur.

Per maximam longitudinis partem cylindrum completam, omnino clausim refert, versus extremum tantum externum ita dehiscit, ut valuula nascatur rotunduscula, partem circuitus internam tegens, nomniluil dilatati, nec nisi laxe membranae subjacenti adhaerens, qunm paries extermus hand dilatetur, enndemque ac reliquus duclus arctum cum membraua nucosa servet nexum. Vialvula haec in margine anteriore per quinque, in posteriore nonnisi pere duas cum dimidia lineas a reliquo mealu sejungilur, margine inferiore ductu hatud interrupto in ijsnm transil, nt minine cartilaginem propriam, sed partem tantum duclus sistal. Ino, re proprius inspecta, totus duclus cartilaginens, quamris membrana interua integrum sistat canalem, in medio anbitn interno, excepto forsan parvulo spalio, extremum internum sequente, fissus est, marginibus lamen oppositis sese langentibus, et fissura haec in extremo exteruo nomnisi dilatatur. Membrana interna lugosa, el in fine interno, statim aute membranam tympani cminentiis duabus, sillis allis, magis eliam angustatur.

Sic dilatari alque aperiri aditus ad mcatum cartilagineum potest.

Perficitur antem illius raluulae motns musculo satis forti a musculi culanci, circa aurem externam fissi, fasciculo interno abeunte, aţue margini valsulae anteriori inserto.

Practerea niluil musculosi observare polui. Vix dichn indiget, fasciculos, in puos musculns cutanens hac regione dividitur, ad constrictionem orificii anris externine necessario facere.

Meatum hunc, callilagincem in capitis basi excipil menbrana tympani, maxima, oblonga, extrorsum nomiluil cava, introrsum convexa. Circumdatur parte anteriore el interno et mounithil posteriore, Jic autem arcu osseo maxima ex parte satis crasso, extremo interno sursum directe culem temissimo, ammuli rudimento, nounisi tcla rellulosa laxa cum reliquo capite comexo. Circulus hic parte interna, anlequan retrorsum incurvalur, processus plergoidei extremum posterins allingit, quod ob sententian recentiorum de ossis quadrati natura memorandum est. Pouc hunc aunulum, cum membrama țmpani mitum reperi ossiculum minus, omnino ab annulo separatum, sed circulum clandens, nomiluil magis introrsum, ad perpendiculum positum. Anmulo latius sed tennius est, planum, deorsume angustatum, margine convexo retrorsum, concavo antrorsum spectans. Iloc, ni fallor, pro malleo habendum est, qunm cjus locum teneal, nisi reclius forsan processus syloideus dicilur.

Sequitur magis introrsum, e regione lujns ossiculi superiore aliud, triangulare, ilidem perpendiculare, plus dimidio minus, quod pro incude habeo.

thujns faciei internae el rersus extremum inferius affigilur mobili nexu alind, minus etiam, sine dubio stapes, Inorizonti parallelum. Onnino, columellam reptilem referens, eylindrum sistit, utroque extremo in patellam inflatam, quarum interna, longe majore foramine ovali insistit.

Iranc fabrican (qunn in utroque, quod dissecui, animali viderim, pro constanti labens, cmm claris antecessoribus de hac anatomia Oruithorlyychi parte haud onnino convenire possum. Omures quidem recte columellam dicun, sed cl. Ilomius, describens nomisi duo ossa, et post cum el. Blainvillius nonnisi de malleo et cohmella locpucus, semianmulum practervidisse vel cum codem confudisse videntm. 2). Cl. Rutolphius nounisi columellam et anuulum describens, malleum pro hujus parte, et quidem postica habnit, incudem haud ridit.

Omnes eadem, sed variis nominibus descripsisse, el eo peecasse videntur, quod in magni ossis, annnlnm, mallemn et incudem non referentis tantum sed revera separatos continentis, compositionem haund inquisivere.

Qumm jam cl. Blaimillins haud ineptan labyrinthi el tympani dederit descriptionem ${ }^{3}$ ), miror, cl. Rudolphio cam desiderari risam esse $\left.{ }^{4}\right)$.

Equidem meátum osscunn omnino desiderari miratus sum, statim enim ablato aunulo cum membrana tympani in carmm tympani incidi, quod recte cl. Blaincillins amplissinum, sed minime profmudum dicit. Antrorsum et introrsum dirigitur. In parte extima recessulo gandel, in processimm mastoi-

1) L. c. p. 23

2) I. c. p. 23.

3) L. c. p. 19

4) L. c. p. 11 
demm dncente. Ilic ubi incipit, fenestra adest ovalis, parrnla, hic fere rotmuda, ante ipsam, rersus finem internmm, entunentiola cochleae. Lineae distantia a fenestra orali, ommino retrorsum, ponitur rotmula, ovalem, sed hand multo, magnitudine superans. Conducit ad cochlean, parum prominentem, sed latissiman, introrsum conrexam, extrorsm concaran, circa modiolum flexam, et in scalam supcriorem et inferiorem sueto more dirisam. Quantrm equidem videre potni, ne semicirculum quidem refert. Magis forsan compositam rimari lnand rolui, ne, exitn incerto, nimis capnt deinrparem. Canales scmicirculares liberimi prosilimu, recte quidem monente liudolphio, illis 'T'ilpae majores, sed labita ad reliqum caput totmuque animil ratione longe minores, nt utrumene animal hand conferri mereatur. Celernm canales senicirculares suetum mammalium typum servant. Spatim inter ipsos tricunetrum magnum, et Blaimillio et Rudolphio dictnm, (quoad usım ignotmm ${ }^{1}$ ), lobnlnum cerebelli recipit, et matumalibns fere ommibus, immo homini jureni commune est. Neatns anditorins iuternus et pars petrosa introrsum posila, minima smut.

Aqnacductum restibuli, quem Blainvillius pro ampulla habuisse videtnr, magnum, quamris non ejus, quam cl. Rudolphims dicit, inveni magnitndinis, cocluleac non eruere potui.

Tuba Linstachiu orificio satis magno, nulla ralvula clauso, hiabat.

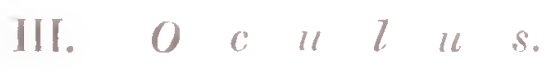

Oculorm simm palpebrarumque aperturam jam supra indicavi ${ }^{2}$ ).

Structuram allins rimando, orbitam a parte superiore atque anteriore, hic cartilagine oblonga mobili, isthue substantia fibrosa perfectius clansam et sic ocuhum melius tectum iuvenimus. Cartilago modo dicla ab extremo orbitae auteriore superiore recta extrorsmm tendit. Fabrica, siue dubio ad Arium el Sauriorum, praccipue Crocodilorum typun accedens.

Palpebra iuferior superiore major est.

Accedit membrana nictilans, duplicaltura quasi palpebrac inferioris, cum transrersa ponatnr, ipsinsque faciem internam tegat. Bulbum fere tolmu obrelare polest.

A substantiate fibrosac, lacmuas explentis, fine postico musculus salis fortis recta descendit, sic orbitam in parte posteriore quodammodo clandens. Afligitur ex parte faciei inferiori, ex parte autrorsmm procedit, et laminae cartilagincae transrersae inseritur, quam nonuilnil deprimere valet.

Sic orbita magis clauditne et oculus melius tegitur.

In palpebris libras circulares tenuissimas iureni.

Practerea dho adsmut musculi distincti et satis magni.

ILorum alter palpebrae inferiori, nec non membranae nichitanti, alter superiori immiscelur.

Ille ante orbitam a facie cranii superiore ortus palpebrae cxtremo anteriori inseritur, illanque antrorsum et sursum trahit; hic, latior, levator palpebrac superioris, ex orbitae fundo recta ad palpebram tendit.

Bulbus oculi rotnndus, trium et quod excmrit in quavis diametro liuearum, hand igitur magmms, ni fallor, nomulla offert, ad ares alque amplibia accedentia.

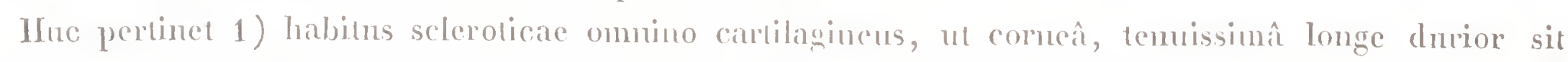
et crassior, neque concidat, hac subito flacesecnte. Falli non potui, nam, quimuis tenuior sit ductu cartilagineo anditorio, reverî hoc multo durioren offendi; 2) relinate summa crassities. Deficit tamen,

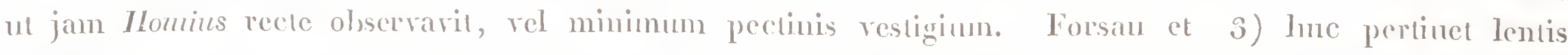
parvitas; frum cnim in reliquis mammalibus fere omnibus maxima ad totum bulbum sit, hic nonnisi dnas lincas alla et lata est.

Satis crassam inveni, qunm diameter antero-posterior unam lineam aequat. Facies anterior tamen fere plana, posterior valde convexa.

Reliquae partes internac nilil iusoliti offerebant.

Mnsculi bulbi oculi proprii crassissimi et latissimi. Choroidea omnino opaca. Pigmentum nbique nigerimum. Corona ciliaris magna. In Iride, pupillan unius lineae diamelti, rolundaur, quae cir-

1) Blainville l. c. p. 19 .

2) Pag. 5 . 
cumdahat, rasa non ad Cetaceorum Plocarnmque tyum eminentia deprehendi. Nervi ciliares siltis magni, sucto cumino mammalium more circiler decem aderant. Nervis opticns parrus, vix terliam lincae partern diametro aequans, relina crassissima.

$$
\text { IV. } N \quad l \quad s \quad u \quad s .
$$

$$
\text { ก. } 22 .
$$

Nares jam in descriptione externa dixi '). Thenul ad carum valde longum, fere toli capitis longitudini respondens, sed angnstum, septo medio in duo dimidia Literalia perfecte divisum. Luitinum utrinsque dimidii latins est el laminis s. valunlis duahns cutancis transtersis hasi iusidentibus, postica margine anleriore, concaro, libero fere ar nariun anbilum posterioren pertingentilus, in meatus tres, superiorem, nedium el iuferiorem, dividitur. Ex lis superior et inferior caeri smut, tres circiter lineas longi, medius in carmm justum ducil, itcrom emineutià longitudinali, inilio latiore, imperfecte in duas, snleo suferno fỉssâ, dein subito augustalâ, maxima ex parte transversì, illic ex hasi, lie ex pariete iuterno enalì, margine libero extrorsum spectante, per duos trientes anteriores quodammodo iu duo dir ismon. Infra partem eminculiae longitudinalis anteriorem foraninulnm brevissino tractu in fratatum dncit, sie satis liberam, nec nisi plicula, qua tegitur, impeditam communicationem inter cavum oris el nasi sigtens. Valvulas modo dietas, animali in fundis paludum victum quacrenti utilissimas esse facile patel, deficientibus musculis, nares claudentibus.

Cartilago, basiu cari masalis formans, per sex linearum spatium ante nares protenditur, weque hic consistit, sed tres ad quatuor lineas lata, dinnidian lıand rrassa, marginem ossis incisivi el supramaxillaris externum tegens, nonnisi lineam cum dimidia pone foranem infraorbilate finitur, sic nerro infraorbitati hasin dans, eumque in stratum superius atque inferius dividens.

$\begin{array}{lllllll}V & \text { I } & \text { S } & \text { C } & \text { E } & \text { R } & A\end{array}$

1. Syslema digeslionis.

Hujus systemalis jam salis accuratae cexstant deseripliones.

Jlomius primo, limgnam bipollicarem, hasi latam, crassim, apice aculam, in hasi dentibus parvis, conncis, aculis, antrorsum versis, pracdilam, papillis parvis, retrorsum repis tectam, nou in rostrum protensam, excepto apice fixam describil. Practer dentes linguales quatuor adsunt, molares, quorum singulae maxillae dnos contiuent, neque substantia vitrea, neque ossea, sed nomnisi connea, libris perpendicularihus constante, compositi gingivis facie irregulari, radicun loco, implantati. Singuli dentes, secundum ipsum, in juniorihns in duos diridi possunt. Inter buccam et maxillas saccus, octodecim lineas longus et lalus, culiculì obductus, porrigitur, in femina, quam dissecuil, substautian quandam concretan continens. Palatum molle valde latum. Oesophagus, puacejue extremo smperiore, augustissimus, vix ventriculum intrane qui dici potest, ut hic, membranacens, potius ipsins dilatatio sit lateralis, qumn duodenum furesse oesophagım sequalur. Ventriculus parris. Intestino temum a crasse nonuisi caceo, pollicen unum cum dimidio longo, quadrantem pollicis rrasso distinguitur. Rectum sensiun dilatilur, el versus anmm rapacissimm est. Opponitur luic par glandularum in maribus longe majorum. Adsumt glandnlae mesaraicae complures, miliares, rasa lactea pauca. 
Ventriculi facies interna lacris, dnodemum vahrulis transversis, reliquum intestinum mullis, contra magno glandularmm numero gandel. Coli inilinum decem lineis longitudinalihus instruitur, glandularum sc. ductibus excreloriis. Cocei canum cellulis componilur.

Mesenterium ot omentmm adipe destitumulur. Hepar quinquelobalum. Ductus cysticus et hepalicns mili, cum ductu pancreatico confhunt, anlecpuan pollicis a pyloro distantia dnodenum intrat.

Pancreas multilobum. Licn duobus lobis, ishmo unitis constituilur ${ }^{1}$ ).

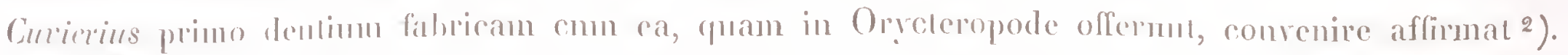
Linguan (pnodimmodo duplierm adesse dicil, alteram in rostro contentam, villis asperan, alteram hnjus hasi allixan, crassionem ol in parte auteriore aculeos duos parros, carneos ferentem s).

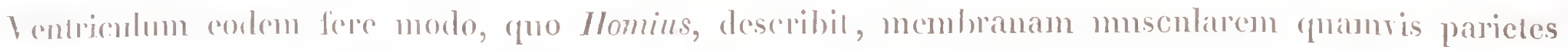

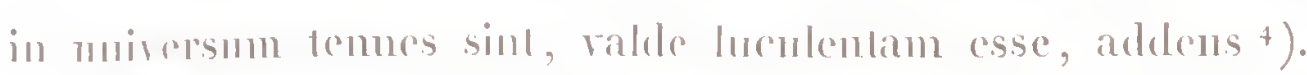

Longitudinem ol ralionrm intestinornm ${ }^{5}$ ) notal. Canalem inteslinalem describens dnodenum reli-

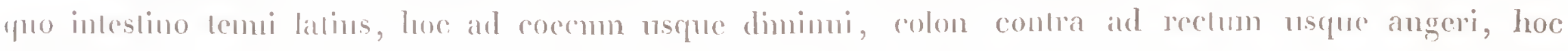

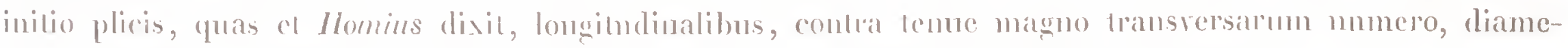

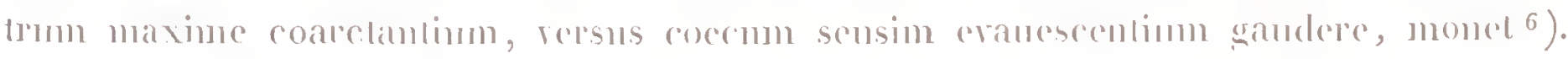

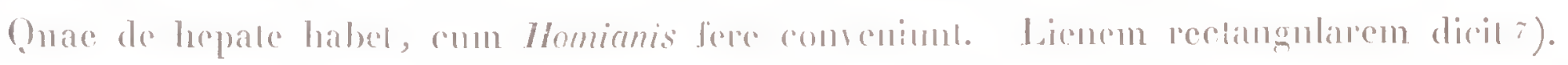

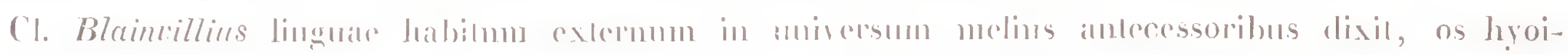

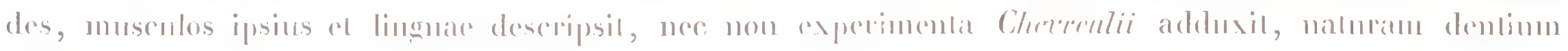
rortucian prohatulia $\left.{ }^{8}\right)$.

('l. Riudolphins dentes anteriores axhibuil'9).

$$
\text { 1). } 24 .
$$

Quar nus conn nudo dirlis ronferenda habemus, haee smul. Primo de musculis manducatoriis,

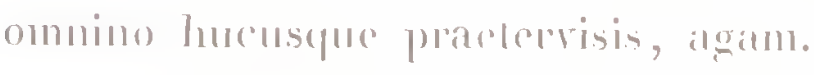

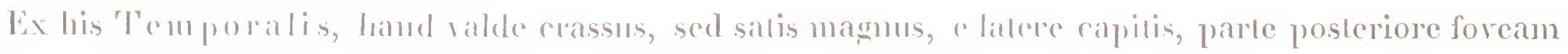

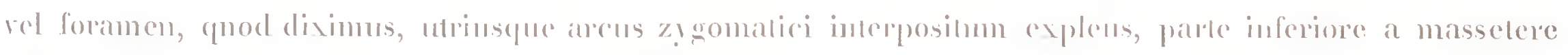
terhus, ad rammon posteriorem maxillare inferioris tendit, et processui coronoideo angusto tendine inseritur.

Mlasseler forlissimus est, triangulatris, a zagomalis farcie exlerma orimulus, a meatu amditorio ad

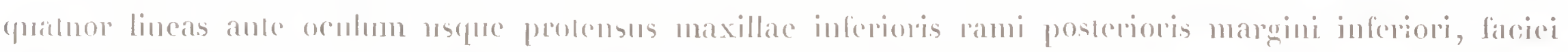

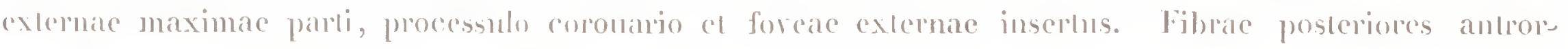
stmm, anteriores relrorsum el deorsum derarrunt.

Lterpue maxillam fortiler elevat.

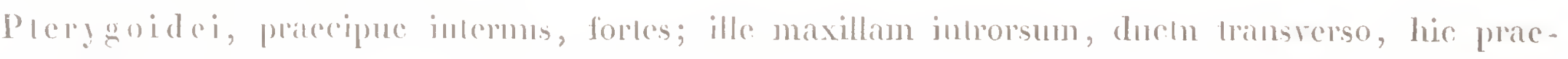
(ipule antrolsm trithit.

Biventer, nl fre in ommihns mammalibns, unirenter, forlis, sed herers, nommisi loli ramo ad secmlemli maxillase inferioris inserilur.

Carmu mis valde fongum, lalum, sed simul a parte superiore versus inferiorem arelissimm, nec:

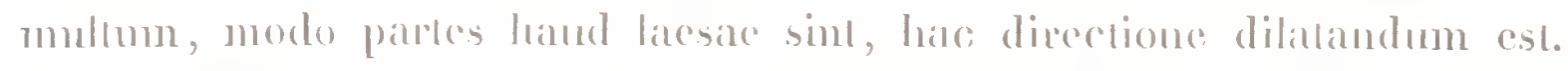

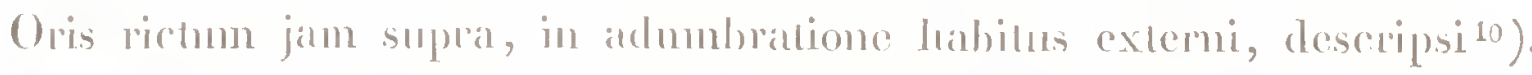

Membrana oris maxima ex parte coriacea el lapris est, fusca, el a membrana externa facici non--

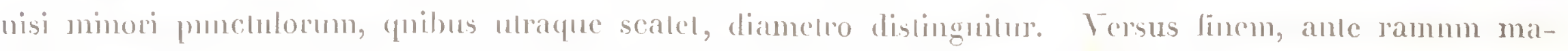
xillate inferioris adscendentem, musculormmque temporalis el masseleris marginem anteriorem, exacte e regione dentum molutum, in sacemn oblongum, circiter viginli lincas longum, quinque in diame-

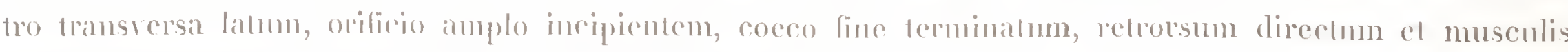
mandibularibus imposilum, cutanen tecomm distendilur.

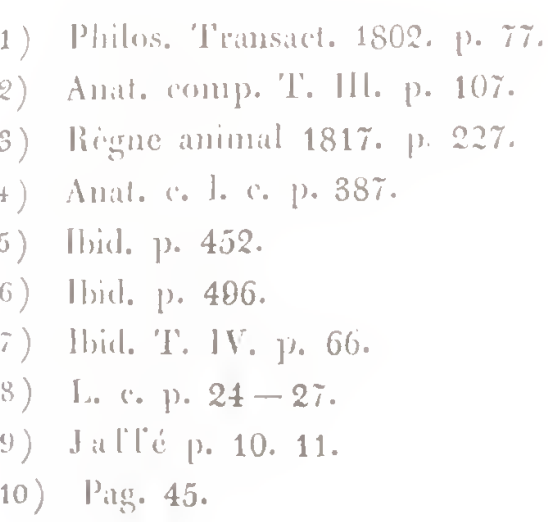


Hoc carnum culicula firma, sicca, vestitur, facile anferenda. Mucosam sequitur stralum musculare, antrorsum ossibus mandibularibus infixum, bucciator extensus. In cavi oris proprii membrana, cacternu

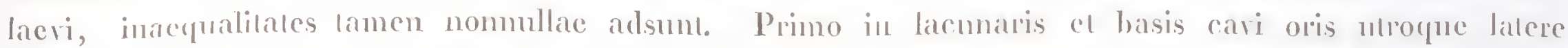
inveniuntur eminentiae longitudiuales, angustae, sese excipientes. Poumutur in secundo fnadrante ante

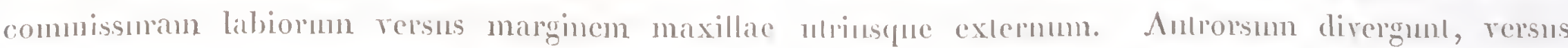

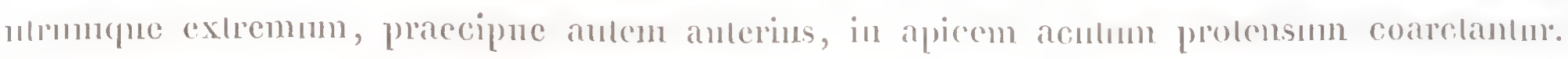

Superior spinam mediam eninentem, per tolan ipsins Iongitudinem, quac decurrit, inferior canm medium, spinam hanc excipiens exhibel. Ad cl. limdolphii mentem pro dentilus sumt habendac ${ }^{1}$ ).

Inter el ante superiormu extrema anteriora contuculiam depressiorem, sed magis composilam inrenis. Componitm duohus cruribus latralibus, antrorsmm divergentibus, el parte media latiore, an-

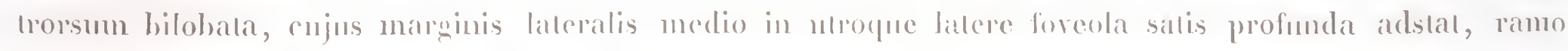
medio et laterali interposita, alyne foranine salis anplo ad cini narium partem anticann ducens. Rano medio subjicitur os incisivum palatinum, cujus of formann refere, lateralibus, mollibus tela cellu-

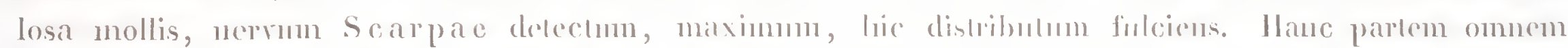

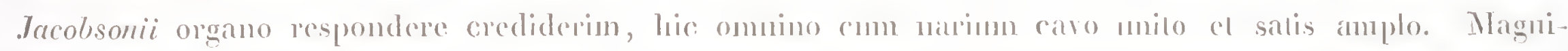
tudo nerornm in papillam hic perera unitormn enorinem aculissmi viri de ejus lunctione sententiae optime favel, (pram is nou cum Blcimbillio omme spatiun intermaxillare hac parte expleri putem ${ }^{2}$ ).

Pone hanc enninentiann, median, inter en pone primo descriptas longitudinales sefmuntur septem ad octo minores, transiersae, hunatae, antrorsmm convexac, e regione extremi anterioris dentimn mola rium desincules.

Jlic elian textura membranae oris mulatur, mollescil el crissessil, praceipue per 5 ad 6 linearum spatium. Simul colore minus obsente finsco gandude.

Per pollicem ullimm demo durescil, sed hic lutenm exhibel colorem.

Simnl per omne hoc spalimn insignilur villis temiloss, primo molliorilms, dein durioribus, quae in ullimi pollicis spatio striis insident undulate transversis, maxime striis, in apicibus digitormm positis, similibus.

\section{j. 25.}

Lingua, nomnisi dimidimn cavi oris posterius explens, in duas partes diridi potest. Mlarum attera, anterior, angustior, sed longior, apice obtuso linita, fere tota libera, altera, posterior, Inevior, sed longe latior est. Sulco transierso profundo ita dividuntur, $n$ posterior trimu linearmun spatio anteriorem tegat. Anterior tegumento duro, inacfuali praedita est. In parte anteriore el posteriore aculeis duris, corncis, retrorsmn flexis, pracsertim in apice, uli linean superant, longis, in media squanis brerioribus et rolundusculis, depressis, pracedilit est.

Pars posterior villis mollibus, lemuibus, slipatis, salis longis insignilur. Margo ejus anterior, supra partem posteriorem proninens, in anenleos tres, antrorsm versos, triangulares, acmos, valde duros,

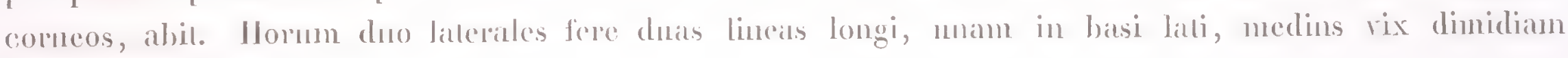
allus et latus est.

Laterales jam Ilomius descripsil et depiniils), num medius semper adsil, necne, ulterioribus indagationibus relinquo. Tabrican constautem habnerin, qunm in ulroque, quod dissecni, animali cadem omnino sil.

Cl. Ilomins hosce aculeos, quos recte corneos rocat, dentibus (qumn contulerit $\left.{ }^{4}\right)$, Blainritlius ${ }^{5}$ ) contra eos papillas alloas molles(que, Curierius eliam carneas ${ }^{6}$ ) dixerint, equidem autem in ulroyne, quod ad mintus est, animali cl. Homium summo jure eos conncos dixisse viderim, farile

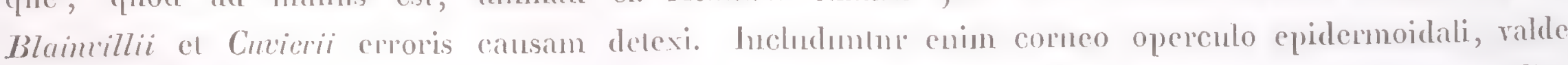
duro el crasso pulpi, 11 ubique solet, altidi el molles, sine dubio in exemplari gallico, deperdito integunento, soli superstites el liberi. Nagna gandentes dnuitie revera secundum Homium ingressum cibornm hand satis diminutorum impedire videntur.

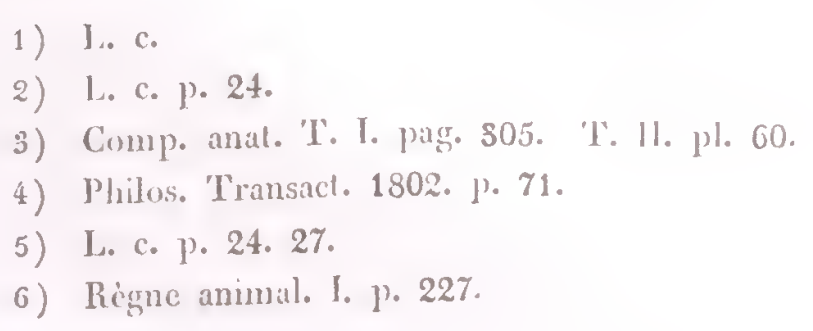


Os hyoides magnum est. Pars media parra, fere quadrilatera. Cormua ejusdem fere magniladinis, sed paullo mijus posterius, retrorsum nouniluil courexum, antrorsum concavum, fiue superiore basi duplo latiore, terminatunn, recta sursum adscendens.

Anterins, anguslins, hiut dilatatum, antrorsum of sursum tendit. Exlremum anterius, parva cartilagine muilur extremo inferiori processus slyloidei, (fuem cl. Blaincillius pro parte cormu anterioris laibuisse vide(un ${ }^{1}$ ).

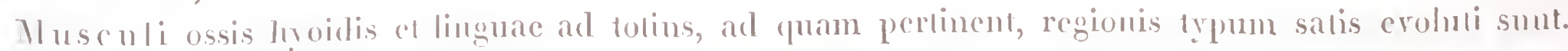
Musculi ossis hrovilis illos linguac at mole el numero vincunt.

Ex illis primus est Omo-mylohyoidens. Hoc nomine venire debet musenus salis longus et latus, a parte scapulac coracoideae el quidem a marginis ipsins superioris dimidio minore exteruo oriundus. Initio simplex, infrat ramo transterso ossis 'Tformis tectus adsectudil, mox in stratum suprerficiale el profundum fissus. Profundum corpori lugódis alligitur, superficiale extrorsum et autrorsum rersum maxillac inferioris faciei internac, panllo ante ipsius extremum posticum inserilur. Quamris musenlus hic maxillas inferiori inscratur, minime hase ipsius pars pro mylohyoideo laberi debet, quum practer ipsum rems my lolugidens adsil, nec inscrtio, in faciem externan facta, conrenial.

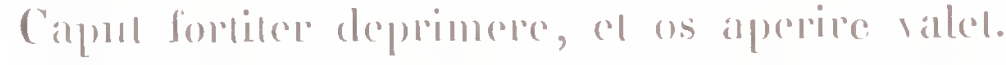

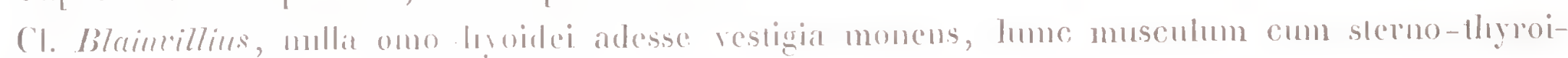

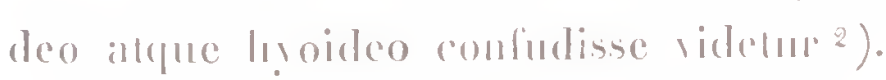

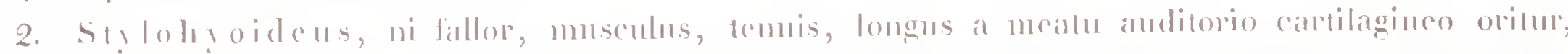

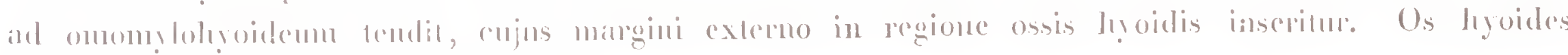

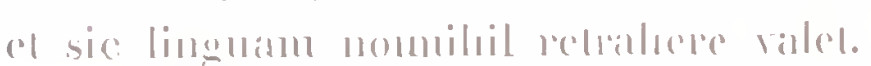

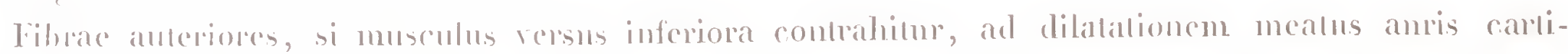

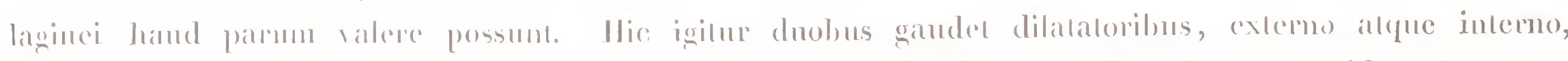

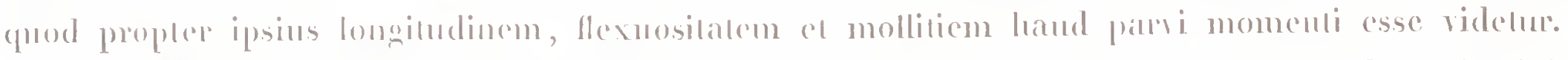

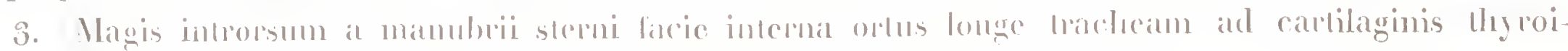

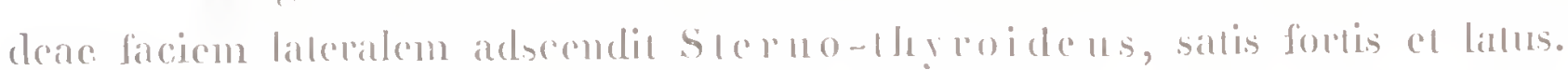

4. Secpuitur introrsmom musculus longe minor, teres, ab extremo cormu posterioris cartilaginis

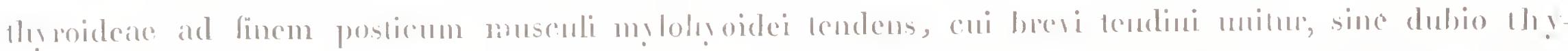

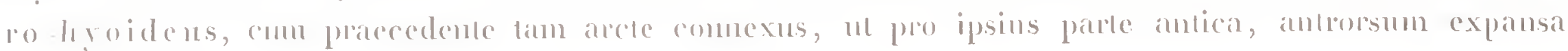
lableri possit.

5. Mylolgoidens, longus, triangularis a fareici maxillae inferioris intermac maxima parte, a commissura seiliect and extremum uspue marminis horizontalis ortus, cum cognomine in linea mediana, crmm modo dicto cellemo postico confluit.

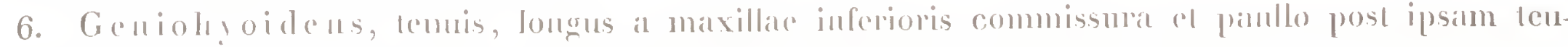

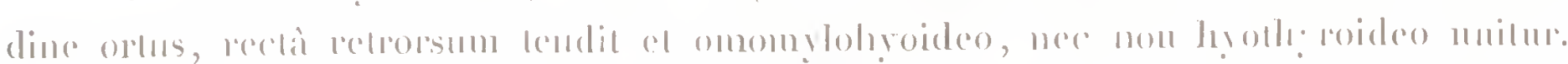

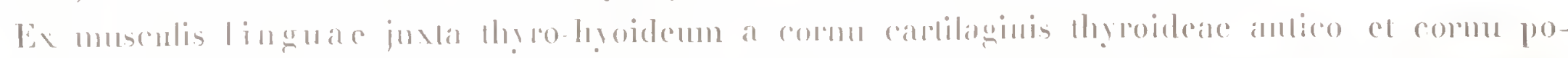

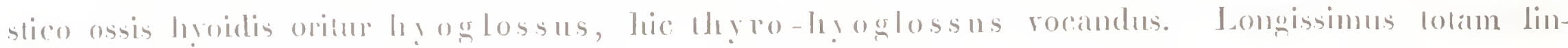

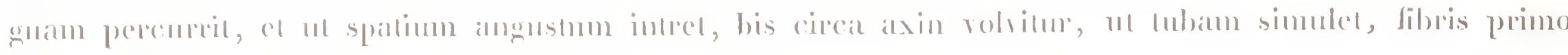
transtersis, sensim fore lomgitudinalibus composilus.

Genioglossus, Irmuis, triangulatis, retrorsmm procedendo intitudine valde auc:tus, a maxillac

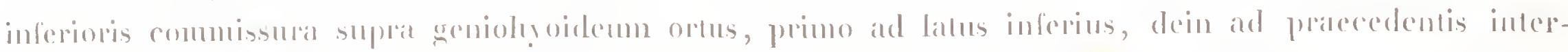
unn posilus, a coguomine luculentissime separatus, retronsum ad hasin linguae silumcue ossis hyoidis medii lendit.

1. 26.

1) entium fabrica onuino cum pracecessorum descriptione in mircrsum conrenit, altamen nomuntla aldjici possunt.

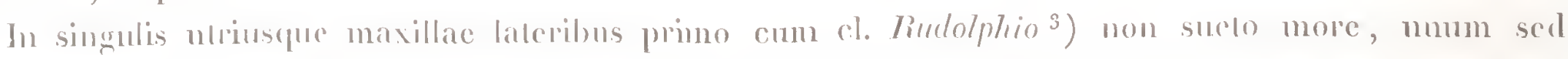

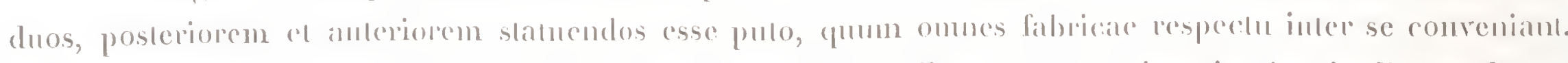

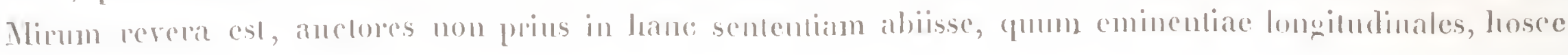

1) 1.. .. p. 27.

2) I. . . 1. 28.
3) I. . . 
dentes quate sistumt, maxime conspicnae siul, el ex maxilla inferiore jam a cl. Homii arlifice luculentissime depiclate 1$)$.

Onmes plani, lommiles, in utroque extremo plus mimusve armminati sine radicibns, facie mandı

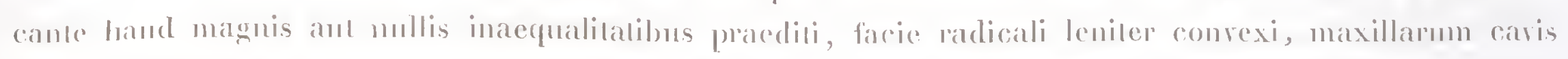
hand profindis insident, el, ubi prominent, gingivis muimlur. Substantia cornea, homogenca compomunlur el per omnen altiludinem canalibus plunimis, minimis, perpendicularibus, rectis ila componmIIr, 11 porosissimi appareanl.

Posteriores, omnino molares, majores, mulcrioribus nomilail bresiores, sed longe linliores sunt. Faries manducatoria leniter concara, in supcrioribus al inferioribus jugis transrersis duobus in tres

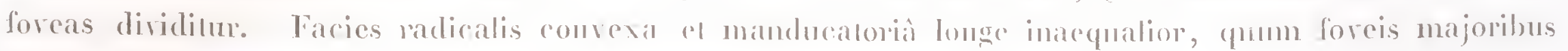
respondeant juga, in tres rel quatuor eminenlias triquellats, satis allats abit.

Forma superiormm of inferiormm non omnino eatem est. llli, nomilnit breriores, sed lationes, margine interuo concaro, externo comrexo, extremo postico latissino, antico anguslissimo, foreis duahus posterioribus, fere aequalibus, antica, longe minima, el minns alla, gantent. Contra hi angusliores, sed nomihil lomgiores, modo ommino opposilo in parte anteriore lationes, in posteriore acutiores sunt el facie masticaloria duabus foreis anlerioribus magnis, postica nuinore instruntur. Hanc fabricam, in ulvigure obvian, conslantem julico.

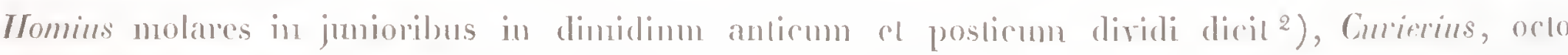

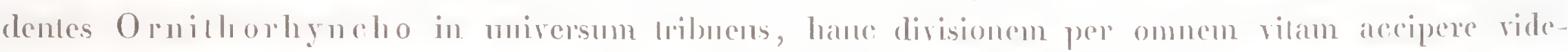

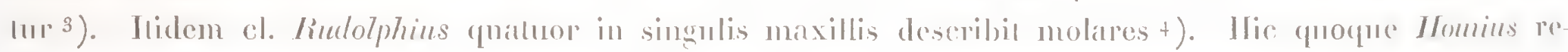
liquis melins veritatem alligit. In mare sce longe adultione, molinem superiorent invenio omnino lan nae non tantum in duas, sed tres partes secedunt, appendicula para, in superioribus antica, in inferioribus

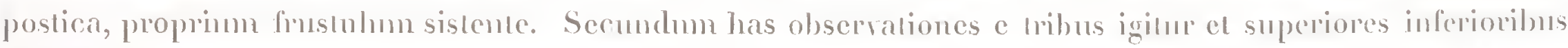
cilius conflumb. In alreolis lincac transrersac, rectae, humiles primaeram divisioncm indicant. Bentes incisivos jam dictos 5 ) nusquam ex phuribus partibus composilos invenio.

\section{§. 27.}

J) glaudulis salivalibus oralibus nonnisi ex parte cerli quid affere possum.

Adest cerlissime glandula submaxillaris, ovalis, laevis, salis magna, pone el infra meatum anditorium carliaginem posila, duchm manifestum ex fine anteriore entillens. Hic mox musculo ge niolyoideo, cui incumbil, techus, sueto more in carmm oris hiat.

Glandula haec dura, mullis lobis componilur.

Practerea alind adest corpus sqanduliforme, duplo majus, magis antrorsmu el cxtrorsmm quod po

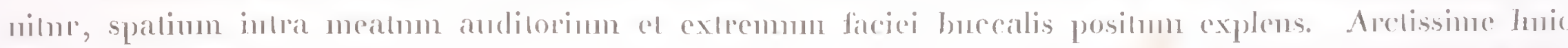

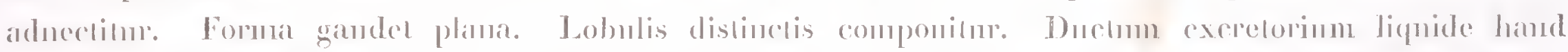
dignosece potui. Hoe num pro glindula parolide an pro strato glandulari faciei buccalis hibeam, du-

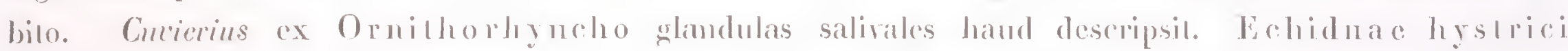
nomisi sublingualem el submaxillarm tribuil. Forma illus, quan sublingualem rocal, rum submaxillari nostra convenil, silus dilfert.

\section{§. 28.}

Palatum molle, fere pollicen dimidimm latum el altum, valde crassum, margine inferiore concaro, in tres lacinias, median el duas laterales, fmubriatas, dividitnr.

Pharyux land ampla, oesophagus haud multo aughstior, ipse angustus, rersus partem posteriorem nomihil dilatatus, sed longus, quatuor cmm dimidio pollires acquat.

Superato diaphragmate subito iuflatu in ventriculum, valde parvm, octodecim lincis in diame1ro longitudinali, decem in transırsa gandentent, sacciformem, longitudinalen. Cardia parum a pyloro distat arcu minore anteriore, convexo, nommisi ser lineas longo.

\footnotetext{
1) Philos, 'Tr. 1802. Tal, I. Fig. 2

2) Plit. 'I's. 1800. 'T'ials. 19

3) A11:1. c. 111. p. 151 .

4) I. C.

5) Pag. 42.
} 
Tunica muscularis tenuissima est, excepta parte pylorica, uhi lincaun aequat, inlima aequalis el laerissima. Valnulae cardiacac nullum, pyloricate vix ullnm invenio vestigium.

Canalis intestinalis tamen statim discernitur el tunicac muscularis crassilic insigni, et valvulis valde conspicuis.

Salis longus el anplus cist.

Longitudo totius ranalis a pyloro ad anum $5^{\prime} 3^{\prime \prime} 6^{\prime \prime \prime}$ aequabal. Ex his intestino tenui $4^{\prime} 2^{\prime \prime} 9^{\prime \prime \prime}$, rrasso cum recto 1' g'" compechnt, ut igitur ratio tenuis ad crassum se habeat circiter nli $4: 1$.

Diancter initio funinque lincas aeqnat, sensim autem in temni infra lujns spatii dimidium descendit. hu colo sensin angetur, ut rersus linem vel dimidinm pollicem habeat. In exilu denuo coarctalur.

T'mica muscularis primo salis rrassa, sensim decrescit, ul dimidium temuis posterius el colon, excepta rloara, nomnisi Iemnissima tunica musculari gandeant.

Canalis intestinalis faciem intcrnam neque crum Homii, ne(ne Cucierii descriptione omnino conrenire vidi.

Secundumn ultumeque in temui transrersae rel circulares adsunt valsulac. Ilomius, has nonnisi in dnodeno, minime in jejnno el ileo orcurrere, haes antem glandulis scatere, perhibet. Contra Cu.

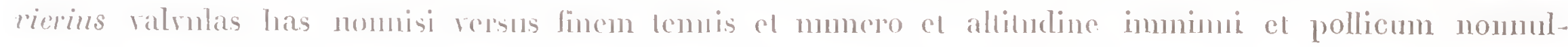
Iormm distanlia ab ejustem comm crasso mione ommino disparese dicit.

Equidem primo fere nusquann valvulas omnino cirentares inveni, sed, exepplo dnodeni initio, satis oblicpnas.

Dein Ilomium omnino el gratissime quidem errasse video, quum non in duodeno tantum, sed in tolo temui vatulite adsint henculentissimae.

Curverius reclius rem descripsit, minime autem valvulis ommino destibitne temuimm finis, sed adsunt, quanvis el numero of magnindine ol directione valde immimulae, omnino longitudinales.

Secundum Homium in crasso decem adsunt lineace longibudinates, punclis notatac; valrula coli adest mulla, Curierius inilio plicas nommullas lomgitudimates adesse, postea cranesronles aflere.

Miror, Momimm omnino practervidisse valsulas, satis latas el allas. Recte pratescntiam puncto-

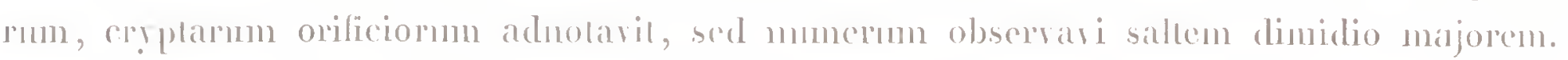

Accuralios descriplio haee fere est.

Per longe maximan partem anteriorem valunlae adsmul, exeepto dnodeni inilio, ubi transrersae pommutur, plus minnsve obliquac. Hace inilio ad duarm linearmm altitudinem adscendunt, maxime ap-

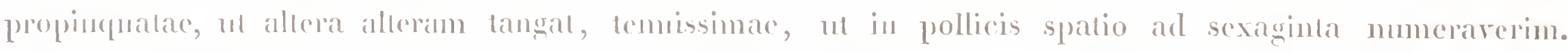

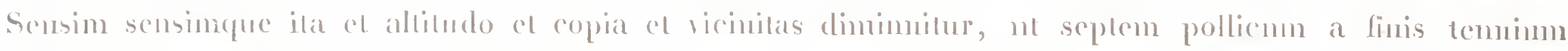

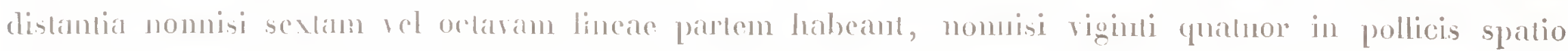
adsint el fere terlia lineare parte ab se insirem distent. Hic subito el ila in longibulinales abemt, ut

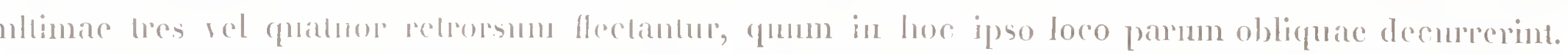

Lnitio creplas mullas meque inter plicas perspicere potni; octo antem circiter pollicum a pyloro

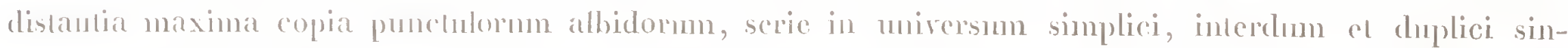
gulis valunlis interpositorum, sine dubio eryptas sistentium, qunn sensim parte posteriore in foveolas atbeant, ilidem plicis inlerposilas.

Plicae longitndinales et in ileo et colo circiter quindecin adsunt, singnlae serie simplici punctorum, praceipue in coli initio distinctornm separalac. In dimidio posteriore crassi intestini el glandulac el plicate delicinut, illis diulius hisce smperstilibus.

In rero recti fine, i. e. supra cloacae initimm superius, subito in utroque latere apparet prominentia oblonga, funlnor sciliret lineas longa, nuam cmm dimidio lata, derem fere foraminibus in ravim recti Jians. Formatur glandnla cjnsdem magniludinis membranac internac apposita, quam pro glandula anali habendam esse, nemo dubilarerit. IIomius hasce glandulas cum glandulis Couperianis, quac practerea atsmul, confunderss, femininas multo minores dixit ${ }^{1}$ ), quod, relictis glandulis Couperianis, minime nalmae consentanemn inveni.

Cuxierius qumm de modo unionis intestini temis com crasso nihil adnotaverit, recte IIomium plenarium valvulae defectmm memorasse invenio. Nullum adest distinctionis restigimm, nisi quod valvulae 
coli forsan nomiluil aldiores et glindular majores et numerosiores adsint. Coecum loco mesenterio opposilo forminine unius conn dimidia lineac e crasso prodit.

Cellusas, Homio in coeco descriptas et depiclas, efpuidem neque in coeco collapso, neque fortissime inflato, neque in aperto invenire potui. Facies interna el externa fere lacvis est; quum autem, re propius perspecta, eminentiae nommllac internac adsint singulares, uhi coecum inflatum exsiccatur, propter distensionem interstitiorum, quippe temuiorum, majorum, cellularmm quacdam species nasci potest. Acque Curierium harum cellularun mentionem iujecisse iurenio.

\section{29.}

II par satis magmm, pondere sex draclumarum iureni ex transrerso ita positum, ul fere șmmetrice per totam abdoninis supremi latitudinem extenderelur, excedente tanen nounilal dimidio dextro. Lobi duo, supremi, ligamento suspensorio magno separali, maxima parte confluchaut, per dimidium anterius fissura profunda ex margine anteriore initium ducente, separati. Ifanc statim in dextro sequebatur forea magua pro resicula fellea. Poue dextrum adscendit rema casa inferior. Sequmunt deorsum lobi duo medii, dexter el sinister, hatius protensi ot longe magis separati, ultimo loco minini, inlimi, quormu sinister, Spigelianns, dextri vix tertiam partem ancluabat.

Textura salis firma. Color hrumens. Biscrimen duarum substantiarum hand detegere vahui.

Vesiculac felleac locum jam diximus. Satis magna est, rohundusculat, octo circiter lineas longa, sex lata. Facies ipsius interna lacrissima est. Buchum lepatico-cysticornm, quamris exacte perpuisiverim, nullum indieimm inseni, sed duchus lıcpatici tres, totidem lineas longi, linean dinnidiam ad unam lati, codem loco ju unum couflunut, gysticmn, ejusden longitudinis, sed latiorem entiltentem. Duclus choledochms satis longns, quum $1^{\prime \prime} 6^{\prime \prime \prime}$ accuet, reclâ descendit ad canalem intestiualem cique quinderim lincarum a pylori distantia inscribur. Lincan fere dianctro acpnat el in decursu parielum vilde angetur crassitudo. Versus finem dilatalur, sed, ubi canalem inlestinalem alligil, quatuor lineas, antegrtam emm intral, valnula circnlari angustatur, quam pars magis etiam dilatala, et papilla brevi, ubi canalem intral, finita, sequitur. Per totum decursum maximo cryptarmm mumero slipatns est.

\section{§. 30.}

Lienem, non ut Curierius dicil, sed, omnino ad Itonii mentem, modo prorsus singulari, duobus lobis compositmm iureni, anteriorem, majorem, el crassiorem, largiorem posteriorem, minorem. Ulerene in hypochondrio sinistro in unum coil angulo aculo. Hics summa lien gandet latiludine, ad polliem fere accedente. Ambo oblique retrorsum el dextrorsmu decurrunt, acminati, dexter demo sinistrorsum et antrorsum reflexus. Satis magnus est lien, lobo antico, dextro quatuor, sinistro, postico duos cum dimidio pollies longitudine metiente. Initio ad sex lineas lati et tres crassi sensim ad duarnm linearum latiludinem et nuins crassitiem decrescunt. Pondus ad drachmam unon acedil, nt ratio ad hepar fere $1: 6$ sil.

Textura gaudet valde laxa, spongiosa.

$$
\text { ก. } 31 .
$$

Pancreas, uli Ilomitus dixil, multilobum, tenuc. Pars ipsins magna, sinistra, longe splenis lohmm sinistrum descendit. Ductus exilis in ampullac, qua choledochus finture, per pollieis spatimm liber decurrens, initio inseritur. Alternm inferiorem, comphuribus animalibus communem, equidem saltem non inrenire potni, nec ah aliis anctoribus traditum rideo.

\section{Respirationis vocisque organa.}

§. 32.

Vix crediderim, hasce partes miri animalis ita esse coustructas, nt admirationem meam angereul, et tamen ila evenit.

Laryux quoad magnitudinem ad mediocres referri potest, et diametro arteriam asperam parum antecedit; fabrica maxime insolita gandet.

Cartilago thyroidea magna, latissima, parum alta esl. Pars ipsins media, acuminata, antrorsum prominel, laterales retrorsum divergut. Sic marginibus definitur anteriore convexo, posteriore concaro. 
Textura maxime memorabilis est, solito cuim more in medio cartilaginca qunm sil, longe maximac partes laterales maxima ex parte osseae sınt. Hoc qumm jan satis mirmm sil, angetur a snela norma devialio divisione utrinsque laminae lateralis osseac in duis, anteriorem et posteriorem, ntramque retrorsmu el sursum directam, quar'mn illa plus duplo longior, hand tamen latior, hanc ex parte tegit. Hace eandem ubique serrat latiludinem, illa versus extremum posterins maxime dilatatur, dein subito introrsum flectitur, et pone pharygen cmm cognomine opposila in linea media fere confluit. Hace pars posterior cartiliginca cost.

Sequitur cartilaso cricoidea, thyroiden ex parte minima tecta, in iuferiore cl laterali circuitus parte altissima, in facie superiore subito angnstissima, 11 hic ipsam el tracheam maximum intercedat spatium, prima multo angustior, marginis anterioris parte inferiore in acumen medium ralde prominens desincute, posteriore panllulum concaro pracdita.

Et in luijns cartilaginis parte media anteriore natnram ossean, mims tamen firman reperisse mili i issis sum.

Cartilagines ar!lachoideac, sucto more tripuetrice, salis magnae sunt.

Epiglollis ex maximis, fulas munpom vidi, latissima, subito acmminala, totam laryngis faciem snperiorem ommino tegens, antrorsmm convexa, reborsmm romeara.

Glandula thy roidea adest, sed minima, in duos lobos, versus facies tracheae laterales positos, vix duas lineas allos, dimidian crassos latosque dirisal.

Prac dixi, ul quoad magnimdinem nelins patcant, mensmam addan. Camblago thypoidea in maxima parte anteriore ol lalcrali lineas duas, in media, nbi retrorsmu flectilur, plus quam quatuor alla cest. Singula cornna sex linearmm latitudine gandent.

Cricoidea in parte inferiore el laterali tres, in facie superiore vix mam lineam Jabel.

Argacnoideac in hasi dnarmm linearum laliludinem habent, altitudine unins cum dimidia gandent.

Epiglottis tres lincas alta et lata est.

Glollis hand valde parra, qunn dnas lineas longitndine aepnet et fere ad tolidem dilatari queat.

Ouam descripsi harmu partium, praceipue autem cartilaginis thy roideac structuram, mum pro nor-

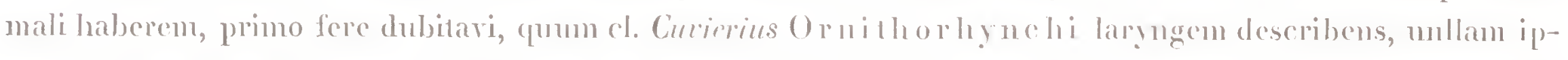

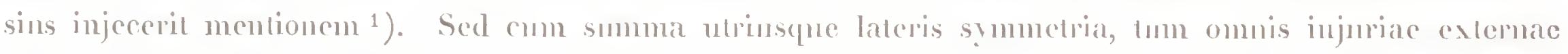
signi absentia primo inpedivit, ne divisionem, quan dixi, fortnito accidisse pulcmus. Neque ossilicationem pro slah abnormi, ant sencenti tribuendo habni, quum animal nostro valde afline, My rmeco-

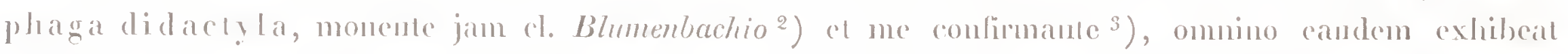
fabricam. Sis jam, ubi prino hare inseni, judicans, postea, in femina juniori onnia cxacte ad cun-dern typum facta videns, vel levissimo dubio liberatus sum. 1)mm haec mendis purgo, divisionem cartilaginis Ilı̣roideate Curierimm serius observasse, gaudeo ${ }^{4}$ ).

Mullum, quac divi, ab iis, quac ab antecessoribus tradila fnere, abludnut.

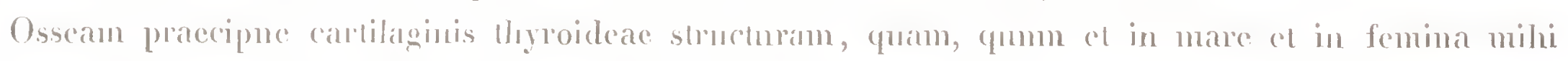
fincrit observata, pro normali habeo, nemo habet. Cl. Blaimillims ${ }^{5}$ ) cartilaginem hane, revera maximam, pro minima luabel, cricoidean recke satis magnam, mims bene ubigne cjusdem fere magnitndinis dicil, pessime cartilayines anylacnoideas parvas et fere rotundas, epighollidem cmm Ilomio, in.

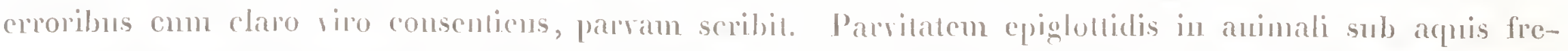
quentissime et din degente et naturae, et reliquormu animalinm codem vitae genere utentiun fabricae

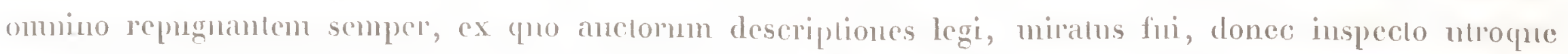
specimine minus bene cos observasse, edoctus fni. Mlensmrac supra datac, errores, hic nescio quo fato commissos, facile probant.

Ventriculım proprimm laryngis, quem cl. Blainvillius dicit, carlilagini cricoideac et ligamento rocali interposimm, equidem hand invenio, quum nommisi solitus adsit, isque parnm profundus.

$$
\text { f. } 34 .
$$

Arteria ispera, ut in ommibus fere animalibus aquaticis, valde latn est, qumm diameter tres at minimum lineas habeat. Eodem modo carlilaginibus componitur primo valde firmis et crassis, ut pa-

1) Anat. comp. T. IV. 1. 510.

2) Vergl. Anat. Ed. 2. p. 281.

3) Archiv f. die Physiol. B3. V1. p. 61.
4) (Issem. loss. V. I. p. 155 .

5) I. c. p. 32. 
rum concidat, dein al!issimis, singnlis lineam in unirersmm of quod excedit melientibus, alque non lambun sese tangentibus, sed mox antrorsmm, mox relrorsmm superscandentibus, ul tnbms inde formenur quasi conlinums, $n$ fere perfectis, nt extrema posteriora vix terlia lincae parte ab se invicen distent. Angelur conlinutas rylindri directione smperiornun saltem annulormm plus mimusve obliqua.

Longiludo duos fere pollices aequal.

Carlilagines muncravi quinderim.

Divisio in bronchos salis alte, dnas lineas aren aorlac allior fit. Bronchi primi proximi, sensim ad latera secedunt, dexter lation et brevior sinistro, funm luic pollicem longiludine, lineam vix unam cum dimidia dianetro aeqnel, ille vix decem lineas longus, contra duabns latior sil.

Sinister ad pulmonem usque, enjus marginem internum paullo iufra apicen intrat, indivisms est; dexter quinque lineas ab hor, loro in tres dividitur ramos, superioren, minimum, lobulo smpremo distinchum, medium maximum, mox demo in duos divisinn, pro secundo el terlio lobo, tertinm, inferiorem, lobum imparem adeunlem.

Fabrica bronchormm usque ad ricinian pulmonm fere cadem ar in trachea observatur, nisi quod annuli nomihil el angustiores sint el unoliores; lic autem el subito nonnilil dilatantur bronchi, ammuli angustantur el natura cartilaginca ommino in osscam mulatur firmissimam. Neque luic consistil hace naturae mulatio, sed per tohm pulmonem bronchus el majores ipsins rami amulis omnino osseis compounntur, ut longe minus etiam concidant arteria aspera el omnino forman cylin. drican rel minores servent, hi quoque dnriores.

Res sine dubio rarias ob rationes maxima allentione digna.

Inter vitae genus et laryngis tracheacque a bronchoomm fabrican facillime nexus apparel, qunm duritie et ossescentia cartilaginum riarmm aërifcrarmm anmalis minaloris mio optime perspiciatur.

Et loc respechn et forsan alio, cognationis se., Barclayi inventum, carlilaginum bronchialium in Delphino albicante ossescentia fabrica hace memorabilis ridelur.

Ulliman ob rationem ossescentia earnulem partinn in aribus compluribus in mentem revo canda est.

Hanc fabricam quum jam ante dnos el quod excnrrit annos in primo animali repererim, de cjus constantia sectione feminae, ejusque longe junioris, omnino persuasmm milhi est. Res omnino eadem est, nisi quod dilatatio bronchormun panlhulım minor, annuli ossei minus duri crassique sint, et ossescenlia mimns alte in pulmones penetrel, quod ulrum ex actate an sexn, an utrâqne causâ pendeal, ulleriori forsan explicahiln indaginc.

j. 35.

Pulmones satis magni sunl, dıos pollices longi, unum lali, quatnor lineas crassi.

Forma gaudent lougitudiuali el, praccipue sinister, planta.

Dexter sinistro el longe major est el dillissione in lobos complunes ab coden, quippe inlegerri1110, dillert.

Homius et Curierius qunm in enmmeratione loborum differant, illo nonnisi tres, hoc quatnor describente, nterqne quodammodo sententiam defendere possil. Adsmut sine nllo dubio lobi tres, optine distincli, maximns, lateralis, totins pulmonis lougitndinem emetiens et solns sinistro major; secundus medius, rel anterior, ante Inıjus parlem snperiorem posilus; terlins inferior, inter dextrum et sinistrum, cor atque diaphragma, nt in magno animalinn numero occurrit, positus. Maximns antem in plures discedere dici polest; nam et extrennm superins, subito inflatum, reliquo nomnisi isthmo jungitur ct inferins rersus mediam longitudinem processum el a me indicatum enittit. Ceterun, quanvis, nt in describendo broncho dextro dixi, pars lobi hujus suprema bronchulum proprinm accipiat, oumes lannen hac lobi majoris appendices substantia pulmonali in unnm ligantur.

Pondıs totins pulmonis, i. e. ulriusque lobi, drachmarum cumn dinidiì dnarum est.

$$
\text { §. } 36 .
$$

Praeter thymum mediocrem, cor tegentem, in Ornithorhyncho alias duas, laterales inveni, satis magnas, exteruas, dorsales. $\Lambda$ summa spinae parte thoracica, nomnisi musculo cutanco et rucullari tectae, antrorsum acmminatae ad brachinm progrediuntur, et inter scapnlam et humerum usque ad thoracis faciem inferiorem pergunt. Color rubicimdus est, structura lobulata, textura satis firma. 
III. $S y s t e m a n r o p o u l i c u m$.

§. $3 \%$

In systemate uropoëtico, omniuo ad mammalium typum facto, renes primo inveni satis magnos, pollicem lougos, sex lincas latos, ad quatuor erassos, margine exterun convero, interno fere recto circomndatos, dextrum nounihil infra sinistrum positum. Facies of ante of post ablatam membranam propriam apparnit omuino laevis. Substantia duplex, externa mollior, spongiosior, fulva, interna durior, lirmior, brumea, facile distingnitur.

Hace papilla nulla, sed facie concara in pelsim hiat. Pondus singulorum renum scrupulum cum dimidio aerpuat.

Glandulae suprarenales non cum cl. Homio parras, sed mediocres dixerim, qunm longitudo ad quinque, latitudo ad tres, crassities ad duas lineas accedat. Substantian homogencaun firmam, molliorem corticali, duniorem internât renum, cari ne minimum quidem indieium inrenio. Dextra magis antrorsum ponitur et sinistrâ brevior, sed crassior est. Glandulae suprarenalis pondus ad tria grana acredere vidi.

Ureteres temues sed longi, in ipsumm urethrae initinm, paullulum intra ductus deferentes aperinntur.

Vesica satis ampla, rotunduscula, nou in pelvi, sed statim snpra ipsimn posita, munica musculari, salis forti, intima praecipue ob multindinem magnitudinennque glandulirum nucipararum notanda componitur.

\section{IV. $S y s t e m a g e n e r a t i o n i s$.}

\section{§. 38 .}

Systema gencrationis Oruithorlyychorum atque Echiduarum unî̀ cum sceleto ad maxime memorabilia ideo praceipue pertinet, pnod, maxime a relipnis mammalibus aberrans, ad rolncria el reptilia proxime accedit.

Lirumrne organon reliquis accuratins ab antecessoribus fuil descriptum.

Ilomius, de organis masculis ageus, externa omnino negal, ul calcar solum sexus masculi reperiatır indicium.

Testes, ratione animalis magnos, in abdomine sitos refert infra renes, epiclidrmidi membrana laxa junctos. Penem hreven, vix erigendum, urinae hand esse pervimm, duplici glinde, papillis quatuor ad quinque gandente, laterali instruchum, eanalem proprium urinam in rectum ducere, praeterea timen, anteynam rectum intret, emittere ductum in pencm, mox in duos laterales diremptmm, singulis glandibus dicatos, per quirtm papillas semen evacuent. I asa deferentia mrethrac ante penis radicem immilti. Vesiculas seminales land adesse ${ }^{1}$ ).

Ductumn seminalium historian iterata sectione probarit ${ }^{2}$ ), partibus genialibus glandulas Cowperianas, quas primo pro analibus labueral, adjiciens.

Organa feminina 1) ovariis valde parris, parum ant non a mammalimm ovariis pecedentibus; 2) tubis; 3) cormubus uterinis, temuibus; 4) vagint, in quam ropma uteri ad latus cervicis vesicae

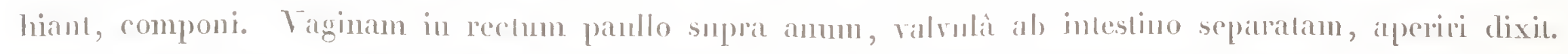

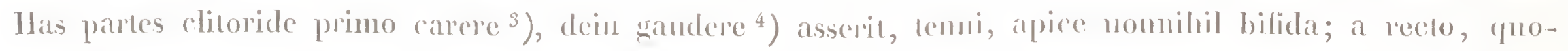

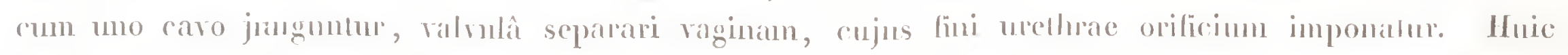
untimque apponi cormu uterinum, unba Fallopiana ad ovirii parvi capsulan conducens.

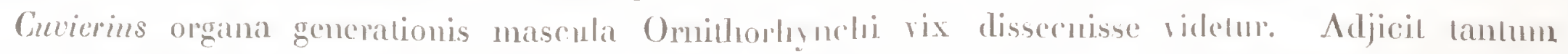
descriptioni genitalinm masculorun Echidnae, quam dedil, salis accuralae, penem nonnisi numero papillarum, Ornithorhyncho tantum dnplicatarum, differre videri ${ }^{5}$ ) et lestes renilsus adslare ${ }^{6}$ ).

1) Phil. Tr. 1802. pait. 1. p. 79.80

2) Ibid. part. 2. p. $35 \%$.

3) Ibid. part. 1. p. 81.
4) Ilbid. parl. 2. p. 357 .

5) Anat. comp. 'f'. V. p. 106. 107.

6) Ibid. p. 15 
Differ lanen omuino clari hujus viri opinio ah Ilomiana de penis structmra. Ounm enim IIo mins el Ornithorlyncho el Echidnae ${ }^{1}$ ) canalem seminalem per penem ad glandes uspue pervium trihuat, nec tambun descrihat sed, saltem ex Echidua, figuris Inculenter exprimat ${ }^{2}$ ), Cuvierius ntrique animali imperviun assiguat, ductibus deferentibns hasi penis immissis, el diserlis verbis negans, se in Echidua iuvenive potuisse canalem, semini propriun atque ad penem ducenten ${ }^{3}$ ).

Descriptioni genilalium femininorum Iromianac nilıil adjecil, clitoridis praesentiam, jam ab IIomio serius coucessaun, discrtis verbis negans ${ }^{4}$ ).

Hanc cl. Blaimillins in descriptione partium, ynan dedit, generationis recte admisit ${ }^{5}$ ).

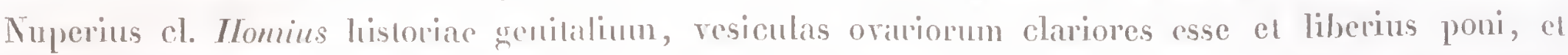
in omuibus, quas dissecuil, feniuis, vesionlas nonnisi in sinistro ovario se vidisse, adjecil, quo similitudo inter (Oruilloplyuclum el Ares liat ${ }^{6}$ ).

\}. 39.

Equidem, genilalia mascula, quae sola perfecte dissecare potui, destructis et ahlatis maxima ex parte frmininis, hoc modo formala iuvenio.

Testium, epididymidum el ductus deferentis silum atque decursum jam supra in describendo partium abdominalium situ dixi ${ }^{7}$ ).

Fabrica hace est.

Testes, pro ratione totius animalis salis evolnti, ad scrupulum singuli pendentes, magnilndine el forma haud parum differuut. Qum enim dexter, valde ungiglus, fere pollicen longindlue, dimidinm

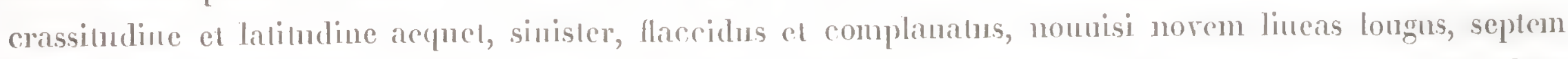
latus of duas crassus est. Hoe per se haud ralde mirum, hic autem ob Homii observatum, secundun quod codem tempore numu tantum ovarium fungilur, forsau memoratu dignum.

lixtremo ipsius superiore duchus excretorins valde flexuosus emergit, primo per nomunllarum lincarym spatium sursum versus, dein introrsum et retrorsum in pelvim descendens, antecpuam in cavum descendal, demuo subito per tres lineas sursum flexus. Per totum lıoc iter peritonaei parieti posteriori duplicalura perilonaci, duos tere pollices longa, sex ad octo lineas lata affigitur. Haec duplicalura a ductu excretorio ad teslis ambilum posteriorem transil, pro distantia utriusque latissima, ut revera omentis adnumerari possil. Qunm enim testis fere ab epididymidis flexura excipiatur, liganentum loc ad duorum pollicum latiludiuem et longiludinem erolvi potest.

Et quoad hane parten idem discrimen, quod testes obtulere, inter utrumque latus obtiuel, quum duclus sinister flaccidus, collapsus, dexter turgidus, teres, hic etiam illo major et flexuosior decurrat, ille cuim flexibus minoribus, hand evolulis, quatuor el quod excurril, hic tres tantum cum dimidio pollices habel.

Testis epididymidisque structura nilil jusueti refer. Duclus seminiferi tenuissimi, iu apice testis antico in ductus efferentes nommullos coëunt. Epididyuis maxime couroluta, nu, heue eroluta, dnos ad lees pedes longiludiue aequarel. Flexurae sensim diminumulur, et per decem rireiter linearuu trachum duclus deferens ounimo reclus descendil, ex tunica crassissima musculari, teuui, sed luculculissima, mucosa composilus. Inseritur initio nethrae ad externum meterum lintus.

Tesicularum seminaliun el ego ne vesliginum quidem imvenire polui.

Extra pelvin ponitur praceipue continuatio cloacac, conica, pollicem cum octo lineis longa, initio duodecim, finc septem lineas lata, et fere eadem crassitudine gandeus.

Circumditur, vel, proad ambitum, formatur musculo sphinctere fortissimo, fere lineam crasso, qui unclio faciei superioris per trimu linearum spatium, faciei inferiori vertebrarum caudalium auteriorum, margine superiore medio fasciculis longitudinalibus, fere duas lineas latis el per totam fere ipsius longitudinem decurrentibus musculi gracilis fün interno affigitur. Sphineter lic in duo strata per majorem

2) Phil. Tr. 1802, P. 2. p. 356

2) L. c. 'Tal), XII. Yigg. 1. H.

3) An. comp. 'T. V. p. 105 .

4) Ihicl. 'T. V. p. 135 et 152

5) L. c. p. 36.

6) Phil. Tr. 1819. Meckels Archiv f. die Physiologic. Vol. V. p. 419.

7) Pag. 37. 
ipsins longitudinis partem facile dividi potesı, ut nonnisi versus orificimm in unum cö̈ant. Externum ad fasciculum, modo dictum, a canda proficiscentem pertinel.

Sphincterem hune sequitur stratum teke cellulosae slipatae valde laxmm.

Hoc ablato conspicitur penis ipse, magua ex parte glaude valde magna formatus. Antequam acl ipsum convertanur, urellurae isthmus, longus, statim symphysin pubalem sequens, canalem laxum annplum, fibris tennibus muscularibus cinclum sistens, adreptendus est. Ifane secunitur pars subito crassior, durior, veri penis initinm. Hic satis magnus et crassus, in stalu collapso et retracto pollicem longus, quinque in glande, eliam sex lineas latus, tres aluns in glande. Glans, dimidimm penis anterius sistens, quadrilatera, suleo longitudinali, medio, in facie superiore alcue inferiore notatur, quo in duo dimidia lateralia dividitur. Tota ipsins snperficies spinis duris densissime stipatis, antrorsum i. e. ad caput versis tegilur. Praclerea in utriusque dimidii extremo versus ipsius latus externum aculei molliores, sed satis aculi, adsunt in depressione, qui tamen omnino extralii possmul, latentes, lineas circiler duas alti, triangulares. Horum numerus hand exacte symmetricus videlur. Homins cnim, latermu laand mentionem faciens, (pumm in altero quincpte, in altero quatuor adesse dixerit ${ }^{1}$ ), equiden exactissimo examine in meo specinnine in sinistro quatuor, in dextro tres inreni, quod, nisi testicnlorum status nonnisi fortuito el practeriens tantum adfuit, propter oppositionem inter partem internam atque externam forsan allentionem merelur.

Glans per totan longitulinem praeputio circumdatur, cunod canali angusliore versus commune cloacae orilicinm hiat, sic ommino a recto discretum, quamsis ntrumpre systema communi circumdetur sphinctere. Pracputium ipsum in majore parte, extima, lacre, in anteriore, golandi vicina spinis, sed pracputialibus quac breviores sunt et rariores, tegitur.

In penis facie snperiore decurrit muscenlus longitudinalis, imprar, ntroque latere apicibus distinctis a sphincteris strato interno ortus, antrorsm sensim atlematns, glandi facici inferiori insertus, ad penem crigendun fictens. Practer lune musculum alins adest longissimus, sed temnissimns, a latere supremo caudae ortus, cirea vasa cautalia et musculos cautales deorsum flexus, el stratum modo dictumn, urethrae extremo, posteriori, uhi in penem ahit, insertus, urethrae dilatator, perinaci, ut ridetur, transtersus, nomilial loco mntalus, et quoad animal maximus.

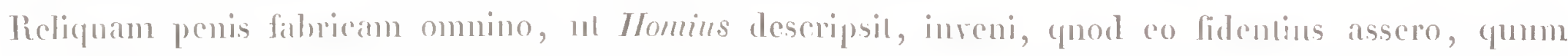
primo ot Curverii anctorilas, ex experimenta a me facta omnino in Galli partes me secedere suascrant. Aperto enim pracputio, el sic glande ipsinsque papillis, quas, ut melins omnia sucecderent, protraxeram, acuam urcthae isthmo tubulo immisi. Hace, omni, qua polui, ri impulsa, minime papillis cxiil, sed, primo recto maxime extenso, per anmm exsiliit. Pluspuam vigesies loog experinento eodem suc--

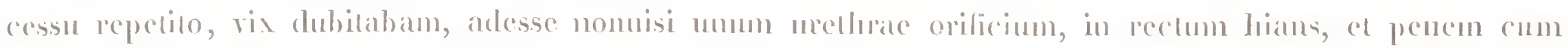
glande inperforatum. Impedichat tamen, ne omnino hane adoptirem sculculiam, pracpulii orificinm, ab) hoc omnino, ul el dissectio et experimentum modo dichm suadchant, separatmm, el, facile aperturam angustam ol forsan fluido coagulato aut plica obstructan vel actuac, praceipue viam latiorem invenienti, resistere posse, simul smspicatus, injectioni dissectionem adjeci et resera in urethrae isthmo extremo inferiore pecessmm invent, orificio angustissimo ipertum in canalem, pere penis faciem inferiorem mediam decurrentem. Canalis hic lineare circiter diancter gander, per totan feere penis longitudinem simplex, nomisi in media salande in ramos duos dividitur, ntrique glandis dimidio proprios el per ip-

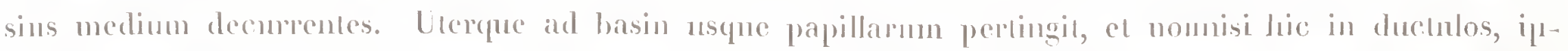
sas ad apicem nsspe percurrentes, dividitur. Quam primum canalem inveneram, mercurium injeci, el repetitis experimentis emm ex papillarmm apicilons exire vidi.

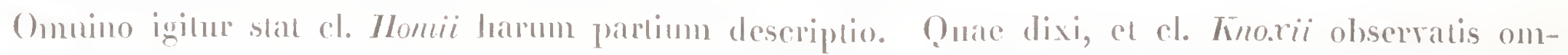
nino conliomantur, ut tres jam lunie partimm statui faveant ancluritates, nec dubito, alios quocue, inter (pnos praccipue cl. Rudolphium, ut manns operi addat, rogo, cadem esse confinmaturos. Quac Cunerius protulit, revera nonnisi at Echiduen pertinent, sed Blaindillii dissectione cjusdern innimalis, quamvis imperfecta, inliemarri videntur, ynum el. vir urethrae seminalis, per penem prodnetae restigia satis lnculenta sibi invenisse visus sil. Minime lamen ipsi, defectum aperturae isthuni medtrace in rectum asserenti, assentiri possmm, quum al Iomii el Curierii ejusdem anmalis descriptio, el Ornithorhynchi fabrica luculentissime demoustrata, contra ipsum fortiter pugnemt. Miror practerea, cl. virnm non ani-

1) Philos. Transact. 1802. p. 80. 
madvertisse, ipsins opinionen valulopere infirmari hahitu partinm femininarum, confirmari antem descriptionem IIomictnam, qum etian in fenina codem omnino modo clitoridis pracpulii canalis adsil, a raginas orificio separatus, uterque eodem exacte loco ae urethra et praepntium maris in cloacan aperlus.

Coternm penis robustus, crassus, membrana fibrosa forti circumdatur. Interior ipsins structura spongiosa, sed densa est. Septo fibroso in dimidia dno lateralia dividitur, quae singnla vasis latissimis peremrnutur. Corpus penis antrorsum fine obtuso terminatur. Hic urethra finditur in ramos duos laterales, grandis dimidium pereurentes. Ipsi tela cellulosa laxa et praepulio, ad medium peuen adscendente tegintur.

Parli penis supremac, anticae, inter ipsum, pubis areum el femoris faciem internam maxime superlicialis ponitur glindula oblonga, sine dubio Conperiana, pro ratione animalis maxima. Semipollicem longa, quatuor lineas crassa et lata, dhplici componiur substantia, exterua, muscnlari, lincam crassitie vel acepuante, vel superante, interna, albida, glandulari. Hlace carmm manifestum, sed, habila totius organi magnitudinis ratione haud amphun, circumdat, orificia circiter duodecim dnctulorum strati glandılosi excipiens.

Ductus ejus, satis amplus, pollicem rirciter longus, flexibns variis in spatium satis breve reduclus, fundum, quo urethra seminalis incipil, penetrare videlnr. Doleo antem, me insertionem eormm non omnino exacte perspicere potuisse, qumm ductus ad ipsam cormm terminationem in perquisitione dilacerarentur. Ifydrargyrum quiden ad urethram usque penetravil, nou antem ipsi immitti polnit.

Maximas revera hasec offendi glandulas, nt testiculos dimidios fere aequenl, quamvis non, nti Homius vilt ${ }^{1}$ ), ejusdem observarerim magnitudiuis.

Iromius primo hauc glaudulan cum illì, quam ex recto descripsimus, confudit, cam pluribus in rectun hiare asserens orilicii.s ${ }^{2}$ ), ino partes secundum hanc sententian depingens ${ }^{3}$ ); postea errorem agnorit et in Echidua et in Ornithorhyucho ${ }^{4}$ ), quar rorrectio cl. Blainvillium fugisse videlur, recte errorem, quem ol. Homius primo admiscral, notantem.

\section{40 .}

É partibus genitalibus femininis nounisi partes externas, abruptis internis, accmate examinare me potuisse doleo, quas sequente modo sese habere vidi.

Intestimmn rectum el ragina, infra ipsum posita, per sex ad septem lineas inde a pelvis apertma inferiore progredimutur omnino separati. Thme cloaca incipit, eirciter pollicem longa ct lala,

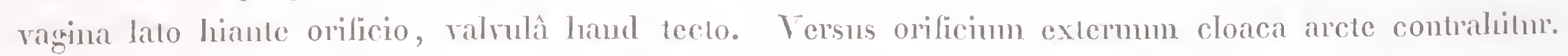

Infira vaginam ponitur clitoris, pollicem ad minimun longa, sed plana et temuis, in glandent, duas circiter lineas longan et latam, itidem plantam, obscure in lobos duos laterales divisam abiens. Hace praeputio includitur quinclue ad sex lineas longo, angusto, ante cloacac parictem inferiorem decurrente, alque sex lineas retrorsum a vaginac orificio in ipso aperturac cloacae margine extrorsmu hiante.

Cloaca musculis satis fortibus praecipne dilatahr. Hormm inferior a symphyseos pubis margine inferiore rectâ ad orificinn cloacáe tendit, lateralis cum caudo-tibiali fine externo unitus a tibiac faciei posterioris extremo superiore transversus introrsum tendit, cum opposito confluens, antecelente tectus el magis antrorsnu parieti inferiori cloacac inscritur, quam dilatare in directione transversa valet.

Huic junguntur fasciculi laterales a processibus transversis vertebrarmun caudalimm tertiac et quartac ad cloacam tendentes, retrorsum trahendo ejus dilatationem hoc sensu efficere valentes.

Antagonista sphincter cloacac crassus et longus est.

Vagina intra pelvim nonnihil dilatatur, el lice pollicis fere spatimm percmrens, $1_{2}^{1}$ pollices igitur aequans et struchram carnosiorem adepta, in summitatis latere utroque orificia oblonga, conrergentia, valvulis haud munita utrinsque cormu uterini recipit.

1) Phil. Tr. 1802. p. 76.

2) Ibid. 'T. 12. p. Fig. 1.

3) Ilbid. p. 356. 'Tal, 12.

4) L. c. p. 30 . 
Cornua modice extrorsum flectuntur et primo vix dimidiam lineam lata, mox ad trium dianetrum extenduntur. Parictes minime temues, sed crassas el carneas iureni.

Tubas cum orariis in meo exemplari abruplas dolens, nomisi pracdecessorum laboribus, jam adductis, uti possum.

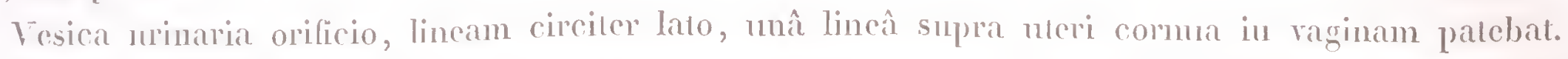

Partem, uleri cormua excipientem, pro vagina labeudam esse, analogia relicpuorum mammalium, pracepue glirinm nonullorum, magis cliam marsupialium, suadel. Imo forsan hunc canalem, practerea nteri cormua et resicam excipientem, non pro ragina, sed cumo labendum, alii censuerint, qunm

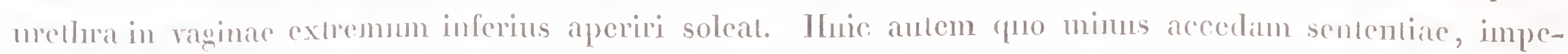
dior urethrae distinctac absentia, quan lie cum vagina confluxisse el ob tunican lujus canalis musculosam pularerim. Sic igitur primo ragina et urethra, dein nterque canalis unitns cum recto in cloacam conflume, ultimo loco accedit clitoridis pracputium. Forsan autem rectius raginac absentia totalis aceipienda, et canalis hoc nomine descriptus, pro urelura labbendus est. Suadel loc exarta ipsins cum urethra virili, respectu structurae el magnoiludinis, similitudo et urethrac in manmalibus lougitudo. Thuc vagiua nonnisi pariete cloacae inferiore sisteretur, aunueute huic sententiae silu orificii pracputialis.

Finctio urethrae, hoc si posncrimus, duplex vix contra sententian afferri posse videtur.

Glandulas, Couperianis maris respondeutes, quîtis data operiì, uti el cl. Blaimeillio arcidil, hand

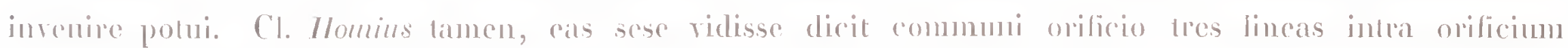
vagiuale in hume canalem aperlats $\left.{ }^{1}\right)$. Si adsmut, longe minores esse debent.

5. 41.

Ornithorh!nchum el Eehidneu mannis destitui, communcm sententiam esse el praccipne Joc fulcro niti opinionem de relegando ex classe mammalium hoc ordine, neminem fungit. Currerius

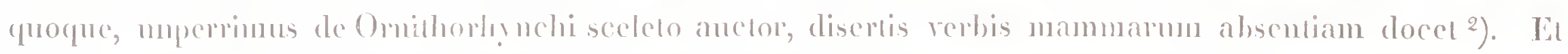
ego, fuamris scdulo marem perserutans, neque papiltarum, neque glandularum ullum restigium insenire potui. Feminam, aperto abdomine el exemptis maxima ex parte hujus cavi visceribus, per tempus satis Jongum de statu ipsins quoad relipua bene certior factus, variis negotiis impeditus hand inspexi, nec nisi rersus anti moCCXXIII fincm, opusculo calecm impositurns, ipsius perscrulationem coepi. Ipso

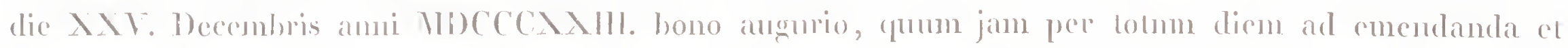
complenda nommulla, ad nervos, vasa, el linguae ossisque lỵodis musculos oculormm aciem inpendissem, acgie ferens, uchula spissa cilins noctem ingruere, ad objecta majora, abdominis musculos, quimsis jann in matre bene visos, memel conversurtus, cutemene cum panniculo carnoso bune in finem

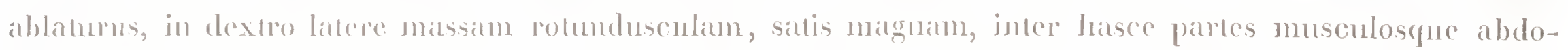
minis positam inveni. Alane primo propter circumvolutim, quam prae se ferehat, speciem pro intestinis, forsan hue pulsis, habni, sedulo autem inspiriens, simnlyue, ul magis certior fierem, latus quoque sinistruin perscrutans, omnino eandem rerum speciem inveni. Tenehris jam ingruentibus, ne

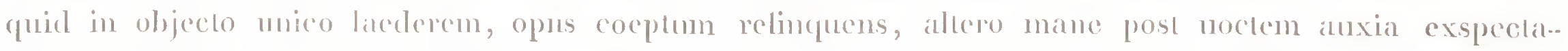
tione insomuem, ad idem regresso, extra ullum dubium glandularis lumjus massae structura posi-

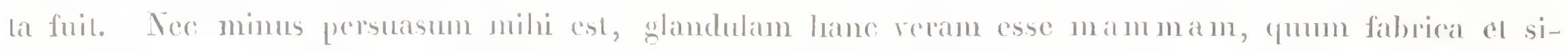
tus, maxima ipsitus in femina crohutio, in mare aut defectus aul saltem volumen ita exigunun, ut neque mune repelita exploratio ullum ipsins dederit indicium, forlissime pro hac semtentia stent.

Glandulae lunjus silus, magniludo el fabrica haee sunnt.

Ponitur in abdoninis latere inter pamiculun carnosum, cui laxissime tantum adlacret, musculum abdominis oblicunn descendentem el musculos femoris anteriores, ad crus uscune pertingens, extremo anteriore ad marginem externum pectoralis perlingens el e regione extreni sterni inferioris linita.

Jam ex lis maxima hujus partis evolutio pated, ut mirnm potius sil, Incuscune can dissectis tot Ornithorlynehis, interque hos compluribus feminis, omnino fuisse practervisan, fuam a me inrentam.

Hated mensuris accuratius conlimantur.

Lougitudo enim accuat quatnor pollices cun tribus lincis, latitudo circiter unum pollicem cum tribus aut (pratuor lineis, reassitics maxima quatnor lineas. 
Forma externa; valde oblonga, jam ex datis dimensionibus patel. Latitudo ubique fere eadem est, sed versus extremum inferins paullulum coarclatur.

Qnoad structuram iuternam componitur maximo numero, ad minimum ad centum cum quadragiula rel quiuquaginta acinorum, cocco fine, paullulum angustato teruinatorum, rersus cutem magis elian in tubulos breves, ductus excretorios, allenualorum. Maguitudine maxine differmont, quum nounulli, lit tamen panci, sex, imo quatıor lineas longitudine haud excedaut, alii, et quidem maxima pars, duos pollices cum tribus, quatuor, sex lineis aequent, unlti inter ulrumque limitem consistant. Lougiores leviter circumvolruntur et flectuutur, minores simplices sunt, majores ex duobus vel tribus, in unum coëuntibus, componuntur. Superiores el inferiores secundum glimdulae corporisque longilıdinem, medii trausversi decurrunt, maximi adscendunt et descendhu, minimi medium glandulac teneut. Omnes laxissine ope telae cellulosac el rasorum, cum acinis decussalormu uniuntur. Primo iuhuilu dissecti solidi videntur, sed perspicillo maxima duchulorum, proxime adstautiun, copia compositos iureni.

Ductuli exeretorii, maxime altenuati, in glandulae medio extrorsum aperimntur. Quamvis neque selas, neque merenrium per ductus, el per se, $n$ mouni, anguslissimos, el spiritu vini contractos, el fluido concreto repletos, trajicere potuerim, area tamen indiratur in cule. Qtuanvis pili hanc partem legant, apparet tamen, hos si abstuleris, plaga, quiuque circiter lineas longa, tres lata, foramiunlis, iis, e quibus pili egrediuntur, majoribus, nigris, circiter octoginta slipata, forsan ductunn excretoriorum orificiis. Practerea in lujus medio depressinncula duarum linearum dianctri adest, pilis destitula, sed emincutinnculis inaequalis, inter quas praccipue una, milii graum hand acquans, reliquas antecellit. Mae sine dubio papillae el duclunm orificia sımt.

Coufirnatur igitur lıoc inveuto asutorum virorum, Okenii el Blaimrillii, sententia, qui, quauris nnuquan Ornithorhynchum feminam inspexcriut, tameu „ob multimodam courenicutiam ipsius cum reliquis mammalibus, nuinime de pracsentia mammarum esse dubilanduu, " isserueraul, el refulantur corum raliociuia, qui ex mamuillarum defectu, forma rostri, ad sugendum haud apta, el oris in orariis el nido intentis, valde dubiis, mammarum defechum deduxere ${ }^{1}$ ).

\section{G $l$ a n}

ई. 42.

Finem opusculi descriptioue orgaui facere lubet, cujns historia aperte docet, longe melins accnrate disquiri, quam ingeniose disputari. De glandula loquor femorali, cui, ne ipso nomine de funclione judicium proferam, forsan retraheudum, hoc nomen a silu impono. Hanc, tolumque apparatum, cujus pars praccipua est, mari lantum couvenire, communis sententia est. Equidem jam antea in codem loco, quo ejus pars exterua, calcarr, ponitur, in fenina foreann descripsi, et postea scutcllum osseum mox describeudum, ad iden systema perlunens, inseni. De glandula autem, quanris sollicite iuquirens, ne vestigium quidem vidi, ul, cam omnino desiderari putarem, nisi tot in animali nostro ad hos usque dies praetervisa, longe majora et magis in aprico posita, suspicionem moverent, dari revera et lumjus partis restigiohum, feliciori forsan fato detegendum.

Glandula femoralis mascula pouilur statim sub musculo cutanco, femoris tolius faciem extcrnau tegens. Triangularis est, sursimn courexa, deorsum concara, pollicem el quod excurrit longa, lineas octo rrassa, tres ad quatuor lata. Pondus duorum scrupulorum inveni. Lacris est, membrana tenui cincta, at lobulis comphuribus composita, firma, fusca. Circa medium marginis posterioris ductus excretorius, primo satis latus, dein sensim angustatus, crassa tunica factus egreditur. Ifunc in utraque glandula omnino simplicen inveni, ut, quam cl. Rudolphins in initio invenil, duplicitas saltem nou semper observetur. Rectâ, flexoribus cruris techıs, pone crus ad plantac extremum posterins descendit, ubi subito in resiculam, duas circiter lincas in quavis diametro meticutem mutatur. Haec toti calcaris hasi adstat, a parte media faciei concavac ossis salis magni, ni fallor, ab ancloribus practervisi, excepta. Ex resicula media duchs, initio fere dimidian lincam latus, exiens calcar iutrat. Hoc ossis modo dicti, satis magui, ad quinque lineas alti, duas lati, in universum haud crassi, sed versus margincm peronealem subilo ad lineam incrassati, leviter extrorstum convexi, introrsum con-

1) Van Hocven L. c. p. 368 sqq. 
ravi, margini externo, i. e. peroneali, hic igitur interno, substantia fibrosa laxa et molli nexu molsili jungitur. Hoc os omuiuo ad hujus glandulae systema pertinet. Primo intuilu in femina ne restiginum quidem ipsins inveni. Rursus antem, majore adlibila cura, in basi cari, cujus supra ') mentionem feci, tela cellulosa ipsi junctam laminulam jureni rotundusculan, tennissimam, lineam et quod excurrit longam, dimidio minus latan, cum eodem musculo ac os in mare descriptum quac jungilur, cidemene ac illud insidet ossi. (unm silus et connexiones omnino conreniant el pro resligio ossis masculi sine ullo dubio habendum est, et optime sententiam meam de vi car plantaris feminini confirmat.

In Monotrenatnun ostcologia jam milui ex Echidua dictum fuit ${ }^{2}$ ). Relicui anctores, quantum equidem video, de eo omnino silent, ut basin calcaris osseam unam tantum et simplicem assumere videantur. In Echidnae descriptione hoc os astragalo insidere, jam monni ${ }^{3}$ ). Hein (l. Rudolphius (pnum calcar calcanco insidere dixisset ${ }^{4}$ ), postea melins, mecum sentiens, idem astragalo infigi scripsil ${ }^{5}$ ). Equidem jam, neulro cálcar insidere, sed cavilate parva glenoidea, in summilate mantronis interui posita, cum ossiculo rolundo, primi ordinis tarsi tertio, mihi detecto, connecli monui. Jloc unm in Echidna adsil, an hice revera os hoc hasilare cum astragalo, uti primo visum futil, nectatur, hujus sceleto hand potilus, nescio.

Mlargini ossis hasilaris interno inscritur tendo glntaci maximi, qno nonnilhil antrorsum el introrsnm trahi potest. Inter idem el plantam tendines flexoris digitorum et tibialis postiei decurrunt, lixi ipso osse.

Ab infinna perones facie postica mnsculus parrns, quadratus, transtersus, ad summum ipsins marginem internum tendil, idem ila introrsum trahens, ul aculeus antrorsmu el introrsmm dirigatur, praceipue antem resiculam comprimens.

Calcalr ipsnm, maxima ex parte liberum, componitur nomnisi substantia cornea, exterua, et ductu membranaceo, idem percurrente, longe minote.

()ssi idcm plano insidere, hoo antem cnm tarso jungi, jam diximus, line Blaimillinm, coutrarimm asserentem, nee nisi ('nli jungi ${ }^{6}$ ), errasse patet.

Ductus excretorii partem extimam nonnisi involucro corneo circumdatan invenio. Cl. Rudolphius

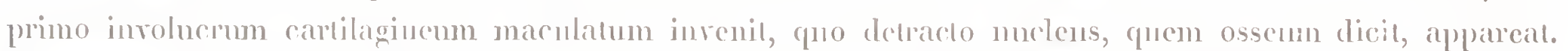
Equidem in meo exemplari, pram is optime conservalo, involuernun illud desiderati, el calcaris partem, basin presse sequeutem, nomnisi culiculae productione, lincam et quod excurrit alta, nunguium modo laxe circundatam inreni. Quum in utroque pede res codem sese habnerit modo, culicula margine arnto terminarelur, sallem in meo exemplanti alia aderat confignnatio. Rem, ut inveni, in fignris expressi.

Leque pro osse, sed omnino pro cormu calcar haheo, qunm fere omnino pellnceal, colore obscuriore, textura minime cellulusa, sed solida, homogene gaudeat, osse tonge nollius sil, ut facile discindatur. Sic rem omnino per tolins calcaris longitudinem el crassiludinem sese habere, repelitis inspectionibus ridi.

Aecque comseninut cum meis, quac el. Rudolphins post prima experimenta tradidit?). Et hice os basilare omnino practeridit, de massa tantum tendinea, cujus ope calcar ptantae insided, loqnens. Calcari insitum dicil conum carmu, ipso haud breviorem, sed multo angusliorem, ragina duplici, interiore, tendiriea, nonnisi inferiorem ipsins partem explente, exteriore, cornea, tolnu calcar cingente, inrolutum. Acque aginam internam, neque externam inreni. Externa nonnisi lanina calcaris extina, vi separala, interna substantia ligamentosa videtur.

Basis ceterum rotunda, phiatuor lineas et qnod excurrit, longa, in medio tres cum dimidia lala est. Dimidio inferiore, exlerno, margini ossis plani interno, superiore, libero, vesiculae impouitur, hic exacte in medio foramine perforata, lincan fere metiente, duclum excretorinm excipiente. Circnitus baseos valde inaequalis est, prominentiis, inlerslitiis angnstioribus divisis circiler sedecim, quibus substantia librosa, interspersis forsan el fibris muscularibus, ab osse plano ad calcar tendens, inseritur.

Ab lala hac basi calcar subilo ad diamelrum dnarnm linearnm contrahitur, et sursum atque in-

1) Piag. 7 .

2) Beil. p. 7\%.

3) Ilint.

4) Al,h. d. Berl. Akadem. 1820-21. 1). 233.
5) Jaflé l. c. p. 14

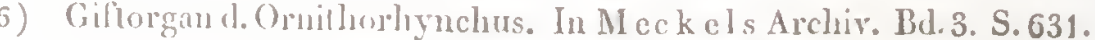

7) Scifert spicil. adenol. Berol. 1823. D. X. Nor. p. 9. 
trotsum directum, ficic inferiore, posteriore convexa, superiore, anteriore concara, autrorsum diriginur et extremo ohtuso lunitur, fere in faciei convexae extremo fissmra parvnla, longindinali perforatnm, iu meo exemplari norem lineas longum.

Camali pereurritur, per dimidium primum, majus ad dimidiam lineam lato, tunc sensim angustato et foranine jam dicto extrorsmu hiante, etiam hand aperto calcare facillime apparente.

In looe canali, exacte enm impleus, sed minime ipsi junctum, continetur ductus excretorii extre-

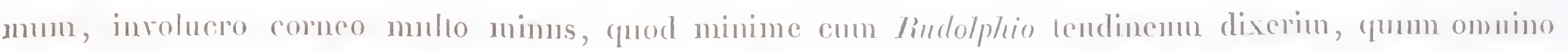
membranae mucosae nolas habcat. In fïne tantum augustissimo subito indurescil, el flavi coloris est, forsan hic corneum el nomilial per foraminulum protraclile.

Hace rera, quantum equidem cumcleare polni, harum parimm fabrica est. Jam cl. Blaincillins, casu, quo vuluns, hoc aculco inflictum, satis gravibus slipabatur symplomatilus, ad carmu investigalionem adduclus, hand optinumu, tanen salis honan, chatems ob desiccationem fieri potuil, declit parlium externarnm descriptionent. Arulei scilicet inrenil canalem, el resiculam ipsius basi adstanten, ceterum aculei fahricam nalurî magis compositam descripsit, sine dubio a corrupto el desiccato partimm slatu in errores compulsus.

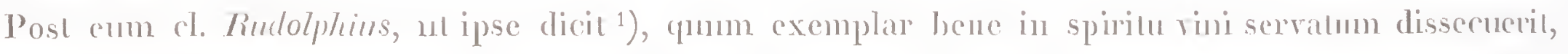
descriplioni Blaimilliance praecipne adjecil canaliculos coecos, a basi acnlei ossei ad ipsins fere medinm decurentes, teudinibus parvis expletos, canalis excretorii, quem teudinemu rocal, in basi fincm coecum, et absoluto Labore Blaimillii seutentiam impugnat, quia historia morbi haud magni monenti sit, veneni expressione risns moveatur, qunm hic niluil comprimi possit, aculens nounisi naribus conreniat,

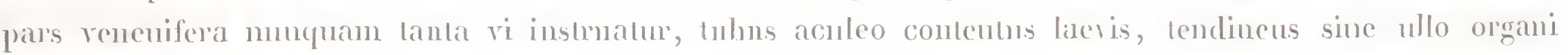
secretorii restigio sil.

Haec argumenta $111 m$ magui ponderis sint, alii videvint. Lubens concedo, casmm, pui perserutationi ansam dedit, per se rem non omnino extra dubimn ponere, quamvis svmptomata minime repugnent, minine anten cum claro auclore 1) expressionem veneni ridiculam dixerim, qum factum tali judicio haud infectum fieri possit, el fubrica, a me reperta, omuino probarcril, cam optime fieri posse, funm resicula, duchus excretorii dilatatio, et fibris muscularibus propriis circundetur, et ita inter calcar ipsiusque os basilare mobile, mobili nexu praeterea cum calcare juncta, pouatur, ut venenum, moto apparatı, exprimatmr. 2) Organi ad sexum masculum restrictio parum ant nihil contra Blainvillium. probat, qunm arma in unirersum maribus ant solis aut majora conreniant, hic igitur solito discrimini sexuali habitus armorum renenatorum accedat; 3 ) ex aliis quocpue organis veneniferis apparatus glandularum serpentimn veneniferarum majore etiam vi polleat, iuo fere exacte, nt jam alibi

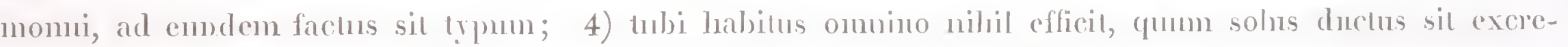
torius, sueto omnino modo sese luabens, el defectus organi sectelorii hand anmuente natura negetur.

Hanc si hene perserntatus fuissel ch anctor, ninine, quanvis ingeniosam protulissel conjecturan

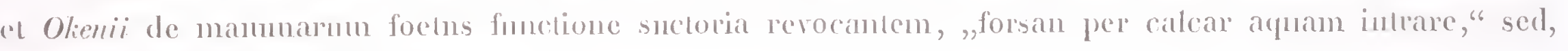
revera ,expelli aliquid" prohassel.

Hoc rerum statu Millius observationem publici juris fecit de tribus gullulis liquoris limpicli, extremo calcaris perforati effucre ipsi visis, el vesicula, quan in calcaris radicis pitrte interna vidil, nec non inter incolas noxian, quanris minime lethalem, masculi calcaris puncturam constare addidis ${ }^{2}$ ).

Equidem ommibus his rationibus persuasus, ol cl. lindolphii observationes nomisi versus amui MDCCCXXIV. linem, omnibus jan din absolutis, perlegens, neque, etsi prius notae fuissent, rel tanli viri sententia a perscrutatione deterrendus, quum resicnlam Blaimillianam invenissem, altins penetravi, et sic ductun excretorinm glandulamque maximam inveni. Hoc mentu ju-

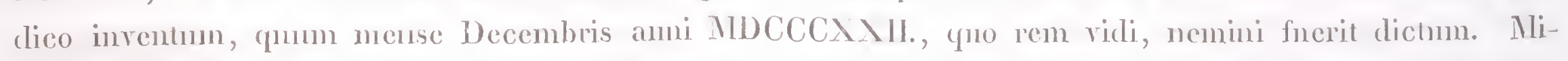
nime corum classi adscriptus, qui, quod videre el quod non videre, magno clamore statim non tanlum sed centies quoque cum orbe literario commmicandum fultant, inventmm nonnisi post quatuor menses, discipulo amatissimo raledicens, nt supras s) nonni, publici juris feri. Quatuor mensibus post, cl. Rudolphius, neque primac ipsius sententiac, neque eorum, quac equidem scripsi, mentionem faciens, anc-

1) Ueber den sogenannten Giftsporn des mümnlichen Ornithorhynchus. In Abliandl. der Berliner Akademic 1820-21. p. 232.

2) Linncaul 'Tr. 1822. XIII. p. 2.

3) P. 2. 
torilate autem cl. TVagneri, modo Londino reducis, cl. Clifiio inventum novum tribucus, primo glan(Lulam descripsit ${ }^{1}$ ), dein eliam depingi curavit ${ }^{2}$ ).

Quo tempore et hie et cl. Ḱnoxius, quem glandulam vidisse cl. Jamiesonii literis d. 25. Julii MDCCCXXIII. ad me datis edoctus fui, rem invenerit, omuino nescio, id autem pro certo scio, me, nullo duce, rel dissuadentibus antecessoribus, observationem instituisse. Anglos, rem eodem tempore vel et postea, ipsis omnino novam detexisse, lubeus credo, qumm dissertatimenlac vix habere polnerint notiliam, quam jussu regio Berolinum missam, examinatorem vix fugisse putabam. JIanc ob rem in cl. Okenii Iside hujus anni intio inveutum mihi vindicare studui. Cl. Rudolphium, ex literis ipsius ad cl. Nitzsclium datis, revera rem ignorasse, postea lactus vidi. 1)e facto, tol obscrvalurihus viso, nullum superesse potest dnbium. Puamvis igitur et nuper cl. Iloevenius in Ornillorhyncho rufo neque foramen externum, ueque vesicnlam, neque canalem, in fusco foramen quidem basilare, minime antem tubum conicum viderit4), hace, ex desiccato lantum partium statu atque incompleta observatione pendentia, nihil contra verum statum probare possıut.

Functionem organi uescio. Aculeus aculus nisi adesset, mere ad generationem pertinere dixerim, el liquorem hic parari, ad excitationem oestri in feminis aptum, forsan plantae loco snpra descripto adspergendum, suadente praecipue Chondropterggiorum analogia, quorum maribus solum convenit fabrica Ornilhorhynchi simillima.

Hanc, autem sententiam vix stare posse, si venenum hac glandula paratur, palel, quum, vuluere facillime inflicto, necessario, secundum omnem analogiam, in feminam quoque noxiferum exseral influxum. Experimentatores, quibus vivorum animaliun copia est, rem facile in apricmm producent. Si onim liquor, el sine punctione nuheri alicui inductus, graviora provocet symptomala, sententia primo loco memorala, ob ralionem adductam, rejicienda videtur, nisi forsan, quod hand pulaverim, alia experientia, veneunm hoc, Oruithorhyuchis ipsis a rulnere applicitum innocnum esse probarerit; contra organon hand vemenifermu dixerim, et mere ad partes genitales pertinens, si liquor ef vulneri arlspersus mulla aul levia produxeril symplomali.

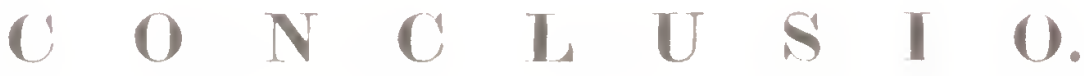

\section{43.}

Pnam dedimus, structurae (Ornithorhynchi descriplio qunm per se, 11 m ad stabiliendum locum, quem in mimalimm serie com Echidnas. Tachyglosso, rence debeal, haud iumbilis esse videtur.

Primo respechu complura puamvis hand ignota fuerint, lamen primo, ni graviler fallor, el haec, opusculo nostro accuralins dilucidalir, dubia, dissentientibus ancloribus, neressario nata, quatenns fieri potuit, sohnta, dein iis, quac jam publici juris fueruml facta, nostris liboribus nora sumt addita. Sic distributio nerrormn rostri, magnitudo quoque nerri divisi jan charomm virormm, Bhumenbachii et Homii disquisilionibns in miversmm patnil; el. Blaimeillins calcar el resiculan in ipsins basi detentam descripsit, nobis anlem mirum nervorum rostratiun complexum el apparalum venenifermm tohm, de cujus parte pracipua, glandula scilice, cl. Blaimillins minus bene dnbilaveral, detegere licuil.

Genitalium quoque masculorum cognitionem complevi el cl. Honii descriplionem contra dubia, quac cl. Cuvierii Echidu a admubratio movere potuit, slabilivi. Glandıla femoralis, qunn jam pars maximi momenti omnino nova sil, laryngis, praecipne carligghis thyreodeae, lronchiornm in pulmonibus contentornm structura ossea, cerebri habilus, mamme el foreolar plantaris in femina pracsentia in eundem omnino censum venimul. 
Practerea sensumm organa, musculorum, rasorum nervorumque decursum fere ommino ignoli exposmi. Ossibus plantac umm adjeci, el calcaris fabricam descripto osse scutiformi explevi. Ossimm, de quibus dubitari polnil, sc. in thoracis regione antica posilormm, significalionem adhibilis omnibus adunituculis rectins forsan antecessoribus stabilivi.

Lacunac, quac maxima ex parte, hand mea culpa, restanl, forsan, si amicorum benerolentia tertio politus fucrim specimine, facile explebuntur.

Ouoad sedem, in animalinm serie Opuithorhyncho, simulpue Echidnac tribucndam, nostra disseclione abunde, ni gravissime fallor, patel, recte a Jonge majore Zoologorum parle Mammalium classi eadem fuisse adscripla, mammarum in femina praesentia omue late de re dubimn solvente. Om-

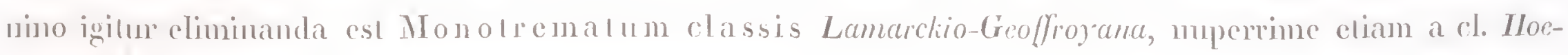
venio defensa, quamis oldin em minime rejicerem.

Nuperime quidem cl. Mocvenins dissertationem suam de Opnithorhyncho conchusione omnino contraria terminavil, Joc antem quum ante inventas manmas fecerit, harum antem defectus praecipuum ipsi sil argumenumu, refulatione longa haud opus est.

Ilos rero invento minime quaestionem de natura parlu producli solit, neminem fingere polest. Generationem Ornithorhynchi multis tenchris premi, nemo ignoral. Nostris diebus testimonia nonumlla fucrunt producta, quibus, emm esse oviparum, probari viderelur. Sic Mitlins, Lirerpolensis, OruiHorlynchum paradoxum feminam dissecaus in ovario sinistro, practer alia minora, ormm pisi magniIndine, flasm invenil, el incolis novac Hollandiae Ornithorlynchum pro animali oviparo, duo ora nido imponente et din incubaute, haberi, ner, non cjus ora societati Jimneance fuisse data, refert. Jamiesomes, dires novac Hollandiac incola, idem sno lestimonio fulcilur ${ }^{1}$ ).

Equidem quamvis fatear, me mullo modo hisce fachis plenarie de ovipara Ornilhorlynchi. generatione convinci, qunm 1) accurata ororum extra corpus inventorum descriptio desiderelur; 2) ex allati.s minime constel, ea revera Ornithorhynchi esse ora; 3) is, qui ovm pisiforme inrenil, mammas practervideril, nec nllo modo pateal, ulrum hoc el reliqua, ab eo risa, rera ova, an polius resiculac Graaflanae rel ex parte corpora lntea sint; 4) teslimonia novae Hollandiae incolarmm vix ullius pretii sint, lamen rem minime negarerim. Contra vero simillima videtur. Quum enim animal nostrum plus ullo alio tres priores animalium classes nectat, et practer alia pracripue genitalimm fabrica ad secumdam terliamque spectet, minime mirandum essel, si et generationis modo eas referrel. Accedit primo, quod discrimen inler partum vivorum foctumn el ororum revera minimum est, nec ullo modo essentiale; dein, (puod, teste experientia, ares fortuito non tantum interdnm ora ita in abdomine incubant, nt foetus viros producant, sed etiam experimenta consilio facta enndem habuerint effectum; postremo, quod animalia marsupialia inter mammalia jam exemplum sistant generationts oviparae simnillinae. Foetus cuint corum quovis respectu tam imperfectus est, ut embryoneun referal vix inceptum. Hace antem animalia

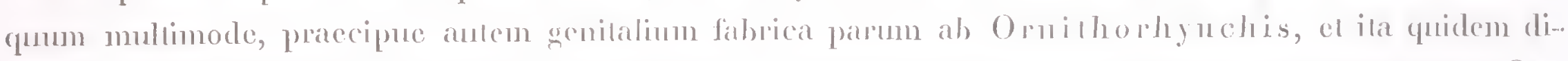
stent, ut hi illis $\Lambda$ vibus el $\Lambda$ mphibiis viciniores siul, valde probabile est, gencrationis quoque modo Ornithorhynchum magis illis ad hasce classes accedere. Sic forsan oripara, simul autem ul Marsupialia, mammis pracdila el lactantia thaec animalia sumb, et jam sic transilus sistitur mulla lacma interruptus a reliquis Mammalibus ad Ares atque Ampluibia.

Doleo quidem, iis quac hic dixi, ab Olienio, omnino contra oviparan Nonotrematum geuerationem pugnante ${ }^{2}$ ), me recedere, sed, omnino cum viro doctissimo de insufficientia corum, fuac pro ororum inventornm natura adducuntur, conveniens, minime tamen in reliefuis cidem aceedere possmm.

Contendit, Monotremata haud esse oripara, quia 1) ex descriptionse Millii paleal, cum non onmm, sed mere vesiculas Graafianas vidisse. Equidem fatcor, ex Itilliana descriplione, brevissima, me hoc minime erueve potuisse, id taulum rideo, el supra dixi, ea, quac affert, non sufficere ad probandam corpusculorum ipsi visorum naturam. Alia Olienii argumenla, ex numero tubarum alque ovariorum duplici deprompla, quum in avibns simplicia sint, nilıil probare, haud opus est ut moncan. Nec video, quomodo ex co, quod in uno lintumn orario reperiebantur resiculac, recte ad naturam mammiferam

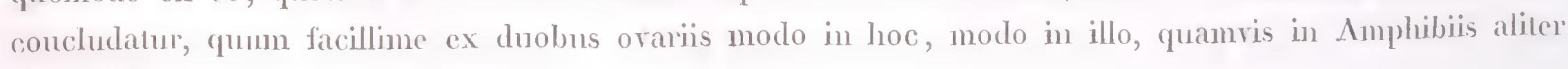
res sese habeat, ova crolvi possint.

1) Transactions of the Linucan socicty. Fol. XIll. p. 2. 1822. p. $622 \mathrm{sq} 4$.

2) 1 sis $1823 \cdot$ p. $1427-30$. 
Pergit cl. Olenins, foctum haud involucris inchusmm nasci posse, quia cx Itillii ororum investi gatione modo irrefragabili pateal, ,ova vitello destilni." Hoc fuomodo ermerit, hand video, nam Ilitlius 1) de ovis extra corpus repertis ommino nihil habet practer nativi novac Hollandiac descriplionem, secundum rtam, ova Ornithorhynchi cum gallinaceis el magniludine cl colore convenimut; 2) de is, quac in ovario sibi vidisse visus est, nomnisi fune supra diximus. Minime igilur, vel vitelli defectus, rel mera humoris limpidi in ovis praesenlia" ex Ilitlii verbis patet.

Hace quum ita sinl, doclissima, quae secpulum, dissertatio de impossibilitate mulritionis ovi ex materia ambiente vix opus fuil; cetorme, ut jam supra momi, lubentissine cl. Olienii offito de natma Monotrematum mammifera accedo.

Supra, gencrationis modo Monotremata transilmm a mammalibus ad ovipara forsan fiucere dixi, non aulem hoc tantum momento haec animalia tres lasce classes jungunt el quodammodo in se unimut. Qnamvis enim et meis disquisilionibns, praceipne antem mammarmm in feminis pracsentia, nti monui, ea ad mammalia esse referenda, probetur, tamen practer genitalia ante ommia sceleton, ob nomnullarum partimn, praccipue capitis el regionis arlus anterioris scapularis fabrican, mammalimm, avium el amphibiorm characteres fere colem mumero affert. Cordis, laryngis et pulmonum fabrica idem probant. Mammarum defochm, rel miniman evolutionem in maribus, papillarmm in feminis, calraris in illis praesculiam, cujus calcar asimm gallinaceorum, ni gravissime fallor, rudimentmm esl, huc quoque referri debere, vix opus est, ut monean. Imo glandula femoralis non lantmm nuanae Sambiorum copiac glandulas ingunales, sed piscinm cliam chondropleregiormm simillimam fabricam in mentem reroeal, ul quatuor reptebratorun classes miro hoe animali alque Echidna mianlur. His tamen ommibus, quum, ul jam supra momi, minime ad formandam classem Vonotremalmm propriam morear, nequidem ila ad separanda hace animalia ab Edentalis compellor, ut in line mammalimm ea-

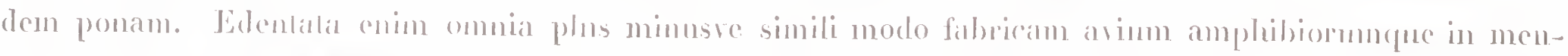
tem revocaut et ipsis 1 onolremata propius acedere fuam reliquis mammalium ordinibus, neminem fugere potest. Iline ordinem Monotrematum, Edentala secuentem, statuendum esse, judico.

§. 44

Onum sic locum, quem Monotremata tencre debent, slabilire studucrimus, breviler tantum quacstionem de differentia specifica ()ruilhorhynchi langamus, ad linem operis consilio neghlectam.

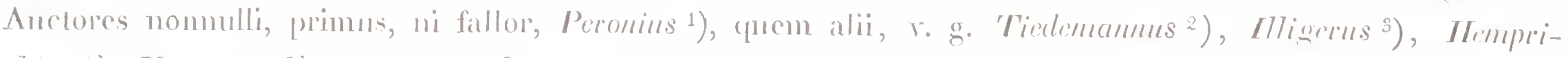

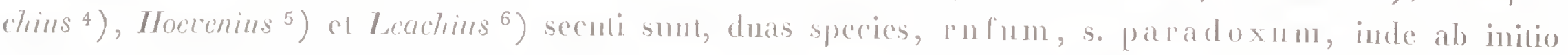
descriptam el disscertam, fusenm, serius detectam admisere, contra alii, pracertim ol. Curierius ${ }^{7}$ ) el Olie-

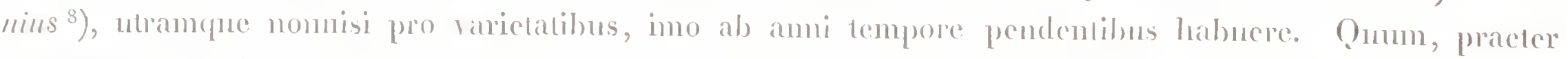

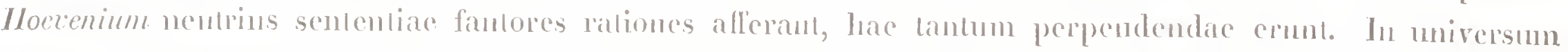
de his monendmm est, characteres, quos alfert, specificos, nomuisi de duobns individnis masculis exsiccalis, in el. Temminctii collectione examinalis, esse desumptos. Iline jure inferimus, quac de rostri et candac forma, nec non diversa pedum alliudine infert, pro maxime ambignis esse lubenda. Neque majoris pretii diversa calcaris ratio ad ungulas videhr, fuam in rufo his majorem, in fusco minorem di-

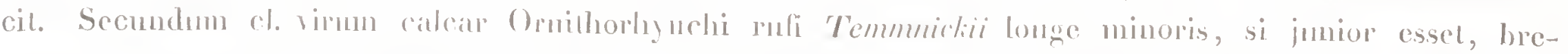
vius, contra fusci, longe majoris, longins foret. Eyuidem omnino contrarim dixcrim, et ex hac ipsa differentia rufum nomnisi juniorem, fuscum adultiorem esse conchuserim, qunm neminem fugiat, partes corneas omnes usn el progrediente aetate diminui, imo eranescere. Neque majoris momenti sunt, quac de epiphysibus Ornillorly nchi rufi, haud in apophyses mulandis, dicil, ymum ipse, nounisi hane speriem hucusque fuisse dissectam, perhibeat, hic igilu sceleli habitus ommino ad genus perlinere pos-

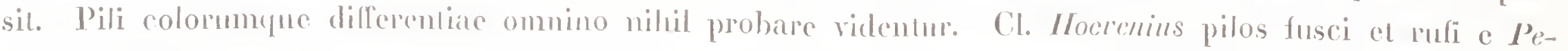
ronio reddidil, ingeme, se nihil ejusmodi detegere valuisse, fatens. Idem jam et ego de utroque specimine jam supra monni. Coloris varielates ommino aut individuas esse ant ab actate vel ammi lempore

1) Voyage de déce aux terres antsales. 'Tah. 34.

2) Zoologie I. 590 .

3) Prodr. syst. Mlimminal. et Ar. 115

4) Grundr. d. Nal.-Ciesch. 1820. 49.

5) Móm. sur le genre (rnithorinque. Nor. Acl. ph. med. X1.2. p. 362. sequ.

6) \%ool. miscellimy II. 1815 .

7) Règne animal. 1. $22 \%$

8) Lehrluch der Zoologie. I1. 957. 
pendere, asserere hand dubito. Ceterum Peronii picturac, praecipue Ornithorhynchi fusci, pessimac sunt, quum color haud fuscus sil, sed fere cyaneus. Adde quod et Peronii rufus longe lucidior sil IIocveniano el specimine siccato, henevole mecum a cl. Nilzschio communicato, contra mea specimina inter haec et IIoevenii Ornithorhynchum fuscum ommino ponantur. Hinc, quamvis cl. Leachius, ut supra monui, colorem Ornithorhynchi fusci melius exhihuisse videatur, de valore hujus characteris valdopere dubilo.

Hace omnia impediunt, quo minus al corum sententia recedam, qui umam tantum Ornithorhynchi assumuut speciem, quac, donec firmioribus aliae probentur, praceunte cl. Blumenbachio, Ornithorhyuchus mihi crit paradoxus. 
T A B U L A R U M EX P L I C A T I O.

TABULA I.

Animalis integri, hand siccati, maris facies rentralis,

TABILA II.

Ejusclem facies dorsalis.

TABLLA III.

Idem. natantis specie a latere visum

TABLLA IV:

Jis. I. Sceleton a facie inferiore visum, qunnm sic optime res maxime memorabiles ob oculos ponantur.

4. Cartilago na:o-labialis. - b. 0.: intermavillare internum et $-c$. extermum. - c. os maxillare snperius et palatinum. - $f$. apophyses pterggoideae, mobiles. - $g$. alveolus dexter. - h. dens molaris sini-ter. - i. cloania. - h. poIns acusticus, in sinistro latere adstant annulus et os posticmun, aut stylum aut mallemm referens. $-l$. scapula. $-l^{*}$. cartilago devtrat inideris. - m. clasicula acromialis. n. clavicula coracoidea anterior. Scapula dectra a facie exterua, reliçna hujus artus onira a facie flecoria spectantur, in sinistro latere al etensoria. Ln utroyne humero tuber", nonbile nexu cunn relipno onse junctum esie patet. Extrenita posterior dextra extelláa, a latere anteriore sinistra, flexa ab interno et posteriore spectatur, nt appareat planta. - p.ossiculum. os calcaris ferens.

Fig. H. Cranium apertum, falcem osseam, perforatam sistens.

Fıg. III. Mlavilla iuferior, cum cartilagine labiali $a$, dente antico, cl. Rutolphio viso, sinitro, $b$, alveolo molar dextro $c$, dente molari simistro $d$.

Fig. IV. Cranii facies posterior, praecipue ad demonstrandan duplicem procensus zygomatici ralicem, superiorem " inferiorcm $b$, unae foranine $c$ dividuntur.

Fig. V. Ossicula ad anditus organon spectantia.

a. externum anticmm, sine dubio anmulıs. - b. externum posticum. Mallens? stylus ? c c et d interna. c. mallent? incens? ntrumene?. al. Stapes.

Fic. VI. Dens molaris ad perpendiculum dissectus.

Fic. VII. Chuae sinistrae facies anterior:
T.IBUTLA V.

Animal a facie ventrali visum, ablatis integumentis et mus culo cutaneo. cujnis partes tantum uomullae, reflexae, a facie interina spectatace ad: cunt.

Varia systemata et hic et in sequente tabula diversis noti indicavi.

$$
O \text { s } s \quad a \text {. }
$$

I. Vavilla inferior. - II. Maxilla superior, vel potins cartilage eam cingeens. - III. Sterni vertebra prima, s. appendix. - Ir. Sterui vertebra secunda. - $r$. Costac cum cartilaginibns. - I I. Osa marsmpialia. - III. Os hyoides. - VIII. Humeriss sinister.

$$
\text { Musculi. Truncus et caput. }
$$

1, 1, 1 etc. Cutaneus, solutus et extrorsum flexus, ut facies rjus interna pateat. -1 **. Musculus risorio comparandus. finniculus, a cutaneo ad os hyoides et lnunc musculnum ten-

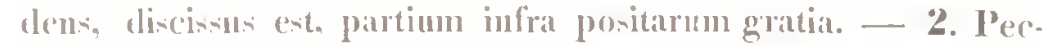
toralis. - 3. Descendems abdominis, in devtro latere integer, in sinistro discisus ot reflecus, nt infra positi pateant. t. Pyranidalis. - 5. Mectus. - 6. Adscendens. - T Tran-rersilw. - 8. Vutator capitis intermus, superficialis, matjor. - 9. Cucullaris. - 10. Omomylohoideus - 11. Siterno-tlyrio-lyo-genio-glosists. - 12. Geniohyoidento. - 13. Stvlo-ligoidens. - It. Biventer mavillae inferioris. - 15. Reetus capitis anterior major. - 16. Levator scapulae. - 17. Obliqunts capitis inferior. - 18. Portio serrati antici unajoris.

F.rtremilas superior.

19). Pars deltoidlis anterior. - 20. Infraspinatus. _ 21. Deltoides - 22. Coracobrachialis snperior. - 23. 2+. Bi(ep brachiii. - 25. Coracobrachialis iuferior. - 26. Ex-

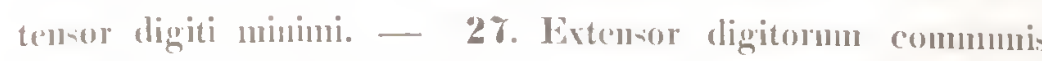
longus. - 28. Indicator. - 29. Extensor pollicis. 30. Radialis longus. - 31. Brachiens internus. - 32. Pro nator teres. - 33. Flexor ratlialis.

$$
\text { Extremilas inferior. }
$$

34. Gracilis. - 35. Sartorils. - 36. Rectus cruris. - 37. Tibialis anterior. - 38. Letentior hallucis longus. - 39. Peronaens Longus. - 40. Peronaems brevis. - +1. Extensor digitorum communis profundn:, brevi analogns. -

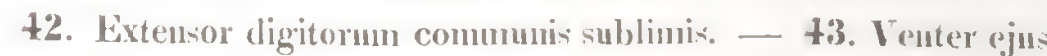




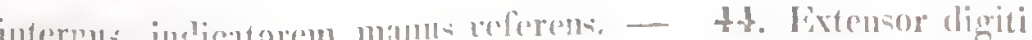

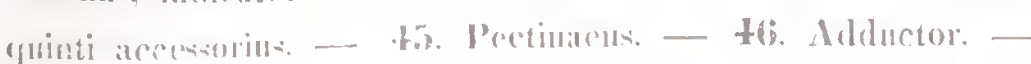

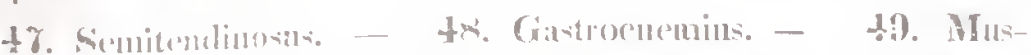

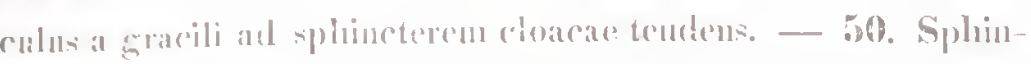

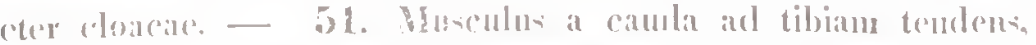

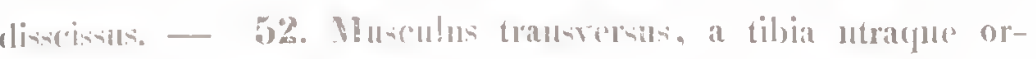

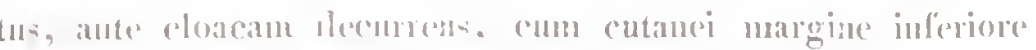

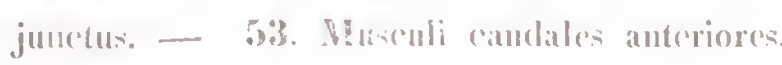

$$
\text { vis cer } \cos
$$

1. Laysix - I. Arteria aspera. - C. Glandula thyroidea. - D. Glandula mavillaris. - L. Glandula pa rotis. - V. Saccus baecalis, in dextro latere clantalle in sinistro apertus. $-G$. VIeatus anditorius. - H. Orilicium cloacale. - I. Corpus caremosmu peris. - hi. Gilamdulate Cowperiauac. - L. Ductus excreterius glandulae femoralis.

$$
N \in \boldsymbol{v}, i
$$

a. Ramuli rami inframavillaris Nerri disisi, habium inlerius

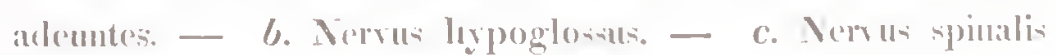
accessorius. - d. P'nenuogatitrichs. - e. Recurrent. f. Sympathicus. - g. Norrus cervicalis tertius. - $h$. Nervis crumalis. - i. Nervi camblales anteriores.

$$
\boldsymbol{V} \text { a } s \text { a. }
$$

a. Carotis. - $\beta$. Jugulanis interua. - $\gamma$. Vasat cruraliat.

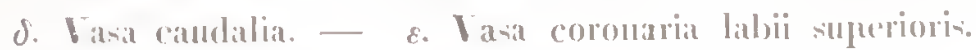

\section{TABULA I.}

Animalis dissecti facies doralis. Ossal hice signis non indicavi, quun capnt ossenn per se patrat, Cartilago seapularis, cujus pag. 13. ot 'Tab. I1. Irig. I. I* mentionem feci, facile ad is perpiciatur. Eandem ols rationem neque nersos, neque vasil literis insignivi, semper offendentibus.

$$
\text { M us culi. Truncus et erput. }
$$

1. Nusculıs cutanens, magma a parte reflecus, - 2. Cucullaris pars inferior. - 3. Cuenllaris pars smperior. -

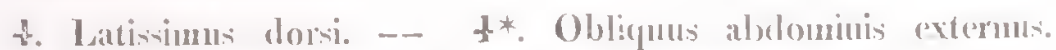
- 5. Rhomboidents. - 6. Seminguinalis et spinalis. - J. Lom-

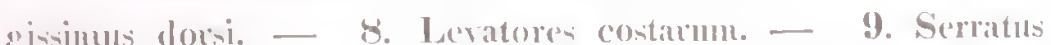
ponterior. - 10. Splenins. - 11. 'Trantermalis cerricis 11*. Vitcouli candace dorsales. - I2. Temploralis.

\section{Fistremilas suprerion.}

13. Deltoides. - 1+. Infiaspinatus. - 15. 'Teres ma-

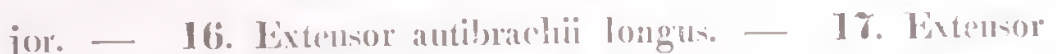
antibrachii extermus. - 18. Flexor uhuaris. - 19. Litensor pollieis. - 20. Extensor digiti minimi. - 21. Flexor digitomn communis. - 22 et 23. Lumbricales, fasciculis tendineis crassis ad membranam interdigitalem tendentes

\section{Firfemilas inferior}

21. Glutacus mavimu, in sinsitro latere quoad partem stperiorem, in destro suoad inferiorem. - 25. Glutacess medius. - 26. Gilutaens unimus - 27. Iliacus 28. Bicep) - 28*. Rectur. Notandum, hic incisotis literarum vitio deene * facile adjiciendum. - 29. Flevor arecrsorius al candla aul tibian tendens. - 30. Semitendinosus. - 31. Semimembranonn. - 32. Tatuls axtermins. -

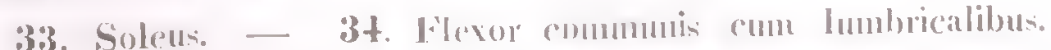
- 33. Peronatens longus. — 36. Evtensor laallucis longus.

$$
\text { I is c c o r }
$$

1. Orificinum narimu sini-trum. - B. Cutis labii superioris reflexa. - C. Neatis auditorius. - D D. Vialsula ad ip-ins initimn. - $\quad \therefore$. Oculn. - $F$. Cartilago ante ocu-

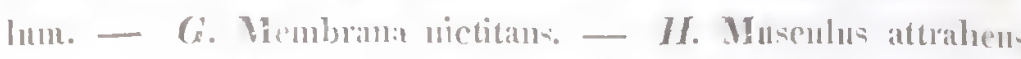
falpehran. - I. Glandula femoralis. - J. Ductus excretorius. - I. Vesicnta iu bari aculei. - M. Ductus acenlemu permeans: - N. Glandula, thymo similis, cucullari et lativima sepposita. - O. Glandula lymphatica humeralis.

\section{TABLLA VII}

Fic. I. Situs partium in collo, thorace et abdomine.

I. Cordis rentrienli cum pericandio, - 2. Ntrinu dertrun. - 3. Atrinu sinistrum. - 4. 4. Aorta cums ramis tribur, ex areu prodeuntibur. - 5. Yena cava dextra sitperior. - 6. Maclem sinistra. - 7. Tena colli transera. - 8. Vena cara inlerior. - 9. Nervi ragi, cum recurrentibu*, - 10. Plexus brachialis. - I1. 11. Nevi plureniei. - 12. 'Trachea, in broncho: divisa. - 13. Pulmo dexter. - 14. sinister. - 15. Oestphlagne. 16. Diaphragna. - 18. Hepar com cretide 18. - 19. Ventriculıs. - 20. Lien. - 221. Hpiploon. - 22. Coеcum cum intertino ileo 23 et colo 2t. - 25. rectum. - 2ti. Testis sini-ter cum ductu excretorio. - 2T. Vesica urinaria cum uretere destro.

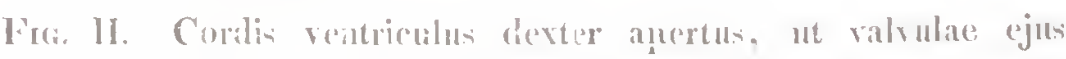
remose structura maxima ox parte carnea pateat. $\boldsymbol{\Lambda}_{\mathrm{p}}$ -

parent simul valualae arteriate pulmonalix.

lis. III-VII. Cerebrum

lin. HII. Facies superior.

Firi. IV. Basis,

1. Neslulla oblongata. - 2. Pous Varolii. - 3. Funi-

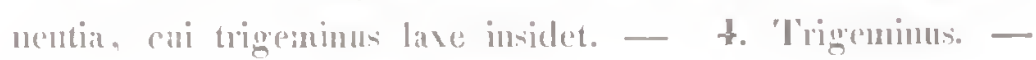
5. Opticus. - 6. Olfactorils:

Ine. I. Ventriculus lateralis sinister, desuper visus

1. Pars melullac cerebri transersim dississa. - 2. Corpus striatuns. - 3. Emineutia quadrigemina.

lis. VI. Facies lateralis.

3 et 6 . nti in Fig. IV.

Fig. Vil. Cerebrum ad perpendiculum linea mediana divismm. 1. Corpus callosum. - 2. Septum prellucidmu. 3. 'Thalinnus. — t. Euninentia quadrigemina. — 5. Commissura anterior: - 6. Nerrus opticus. - T. Eminentia trigenini.

Fici. IIII. Praecipue all organon olfactus.

1. Facies inlerior cutis ficiei dinidii dextri, e sede mota, sinistrorsum flexa, nt pateat cavi narium pars anterior. 2. Nervis ethuoidalis suister. - 3. Nervi infraorbitalis partis superiocis ramus posterior. - + t. Fijuslem anterior. 5. Foramen, quo nerves ethnoidalis dexter exit. - 6. Launina cartilaginea, ossa mavillae superioris cingen- - 7.7. Carmu nasi dextrum apertum. - 8. Parietis superioris sinistronsum retsi facies inferior. - 9. Naris devtra. - 10. Talunla superior. - 11. inferior. - I2. laninae cartiagineae, longitudinales, basi insidentes.

Fac. IX. Lingua cum palato molli

1. fisso, larynge et plarynge. 2. - 3. 3. Pars cartilaginis thyroideae posterior, pharyngen cingens. - t. Cartilagiues ary taenoideac.

Fic. X. Paries cavi oris superior, fars inferioris dextra, cum sacco buccali.

1. Papillat incisiva s. palatina mavinta - 2. Orilicia ad uasum ducentia. - 3. 3. Dentes anteriores. - +. Striat palati transversac. - 5. Membranae palati pars posterior. 
- 6. Mavillac inferion is pars sillistra cum rugis transecris. 7. Saccur: lnecalis apertus.

Fig. XI. Caput. desmper vismun, praecipue ad nervos de sensunu organa. Cranimu apertum, cavi nasalis pars anterior ablatia.

In cranii facie inleriore patent forancen occipitale, ad latera loranina maxima, pars petrosa cum canali senuicirculari superiore et poro acutico interuo, foranen orale ct rotundum, fi-mina sphenoidera, lossa cribrosia in dextro latere. In suistro nervus trigeminus, totum tractum a foramine ovali at fossim cribrosan implens, in ramos tres luculeuter divisns. Ramm: anterior in

ethunoidalem l. et ocularem 2. denno divisn. - 3. Foramen inter radicem superiorem et iuferiorem processils zagomatici. - 4. Arcus zygematicus. - 5. Cartilago et stratum filorosum supra orbitum. - 6. Bavis orbitae, dentem molarem contimens. - T. Foramina uervi ethunoidalis. - 8. $\Lambda$ pertura nasi ositi anterior cum septo. - 9. Os incivirum exter-

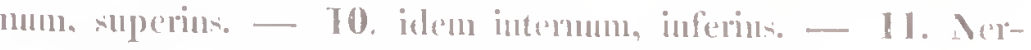
uls septi Scarpae, in papillis palatina diramatu-

Fig. All. Casmu oris a parte inferiore risum, pracripue act yitema nerrominn.

1. mavilla inferior. - 2. Lannina ipsins rartilagines. 3. Pars cutis inframaxillaris sini-tra e secle mota, refleca, ut facies ipsills allperior pateat. — t. Norves inframavillaris

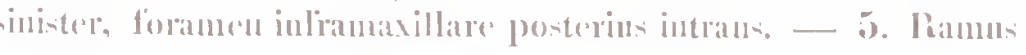

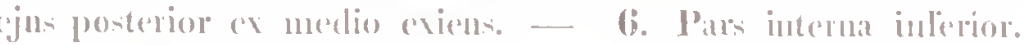
C 7. extermil superior, late praecipme parti sulcatae labii

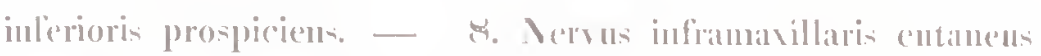

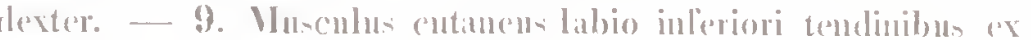
parte fiswis insertus. - 10. Palatum. - II. O) ptergegoideenn, remota membrana palatini apparens. - lo. Ipertura narinu ponterior. at tympana I3. 13. duceens. - It. Heatus anditorius cartilaginens sinister, in fine interno apertus. 15. Saccus buccalis siniter. antrorsum flexus. - $\mathbf{I}$ (6. Condyli occipitales. - 17. Cap-ula articularis communis.

Fis. MllI-XI. Ml comalis intestinalis faciem internam.

Fic. XIII. Jejunum.

Fig. MIV. 'Tran-itus valvularum transtersimu in longitudina$10 \cdots$

Fig. XI. Colon

Fis. XVI. Lachs muionis ilei cum rolo et coecunt.

Fis. AVII-XIX. Larin.

Fir. XVH. Facies inferior s. interior.

Fis. XVII. Anperior s. posterior.

Fis. Mix. lateralis.

I. Epiglottis. - 2. Cormu cartiliginis thy roideae anterim— 3. posterins. - 3*. Lamina snperior. — + Cartilago cricoidea. - 5. Cartilago arytaemidea. - 6. Trachea.

\section{TABILIVIII.}

Fig. I. Genitalia lemuinina.

a. Vesica. - b. I rethro-ragina. — c. Orificia rormum nteri - d. quorum devtrum apertum. - $\because$. Tubate et ovaria - f. rectum. - g. cloaca. - h. Orificinn methro-rat gimar cloacale - i. Clitoris. - i. Pracputiun. - 1. Fins orificiun cloacale. - m. Cloacae orifieimn. - n. Sphincter. - o. Gilandulac anales.

Fis. II-IV. Genitalia masculina.

Fig. II. Totum systema genitale et uropoeticum.

a. Renes. - b. Renes succenturiati. - c. Ureteres. d. Verica. - c. 'Te-tes - f. Epididymides - g. I rethra. - h. (ilaudula Cowperi dextra, aperta. - i. Penis. - li. Glans bifila, crevtis aculeio - l. P'racputimum apertnm. - m. Cloacil apertis. - n. Stratum musculosimm eloacare. - o. Orificimn cloacae. - p. Retractor penis. q. Rectum.

Fig. Ill. Item, exceptis retuibus et ureteribus, canales fere omies aperti.

(1. Vesica. - b. Dustus deferentes - r. Urethra. d. rectum. - c. Gilauthlate anales. $-f$. Cloacae. $-g$. Orificimm nurethate in cloacan hians. - h. P'onis. i. Procputium, protensa glande bilida $k$. -1 . Orificimm praseputii cloacale. - m. Stratun cloacae musculosum.

Fig. II. Penis Tabrica.

a. Pars urethate etima. - b. Meatus seminulis, in r. bificlus. - d. Cilandis dextrae extremun retraetum. - e. sinistrae exsertun, aperto meatn. - $f$. praceputimm apertum a ficie inleriore visum.

Fig. V. Mammae, quarmun dextra

a. refleva cute ommino patet, sinistra nomisi Ioco manmillie b. indicatur. - c. Orificium cloacac. - d. d. Orilicia lovearnu plantarimn calcaris miacenli situm obtine'ntinun. In latere sinistro fovea inversa conspicitur, calcaris cullimentum e funto cxerens.

Fus. Ir. Forea pedis foninini, ossi lenticulari insidens. Vide Fic. III.

Fig. VII. Pes sinister feminims a facie inleriore visns, ad demonstranda antoga calearis misculi.

a. Forca aperta. - b. Calcar in ipsins fundo. - c. foramimulun coecomm, ad calcar tenderls. - d. Ossiculum bavilare. - e. Osiculum pisiforme, oxiculum batilare fulcieas, attragalo $f$. insiclents. - g. coutis plautae, reflera h. plintia. - i. Gilutatens maximus atl plintan, et appanatum calcaris temele'lls.

Fis. VIII-XIII. Apparatus glandular femoralis

Fra. Vhlo. Toths appinatus.

a. Cilauthlia cum ductu, in parte superiore aperto. b. Vericulit. - e. Pirs ductus in aculeo contentus. d. Os calcaris. - e. Calcar apertum.

Fiti. IX. Ot a cum calcare a facie inferiore visum.

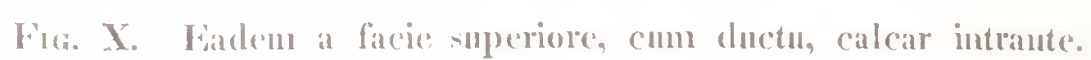
Fig. XI. Facies superior owis calcaris et dimidium superius baseros calcarin, ut pateat modus mionis utrinseques. Caritas, (quo os calcaris unitur cum osiculo pisiformi.

Fig. XII. Bar-is calcaris. In basi calcaris optime apparent for veate, ligamenta recipientes et ductuls glandulae femoralis.

lig. Xlll. Facies interua omis calearic, qua cum calcare IIIIt!I! 



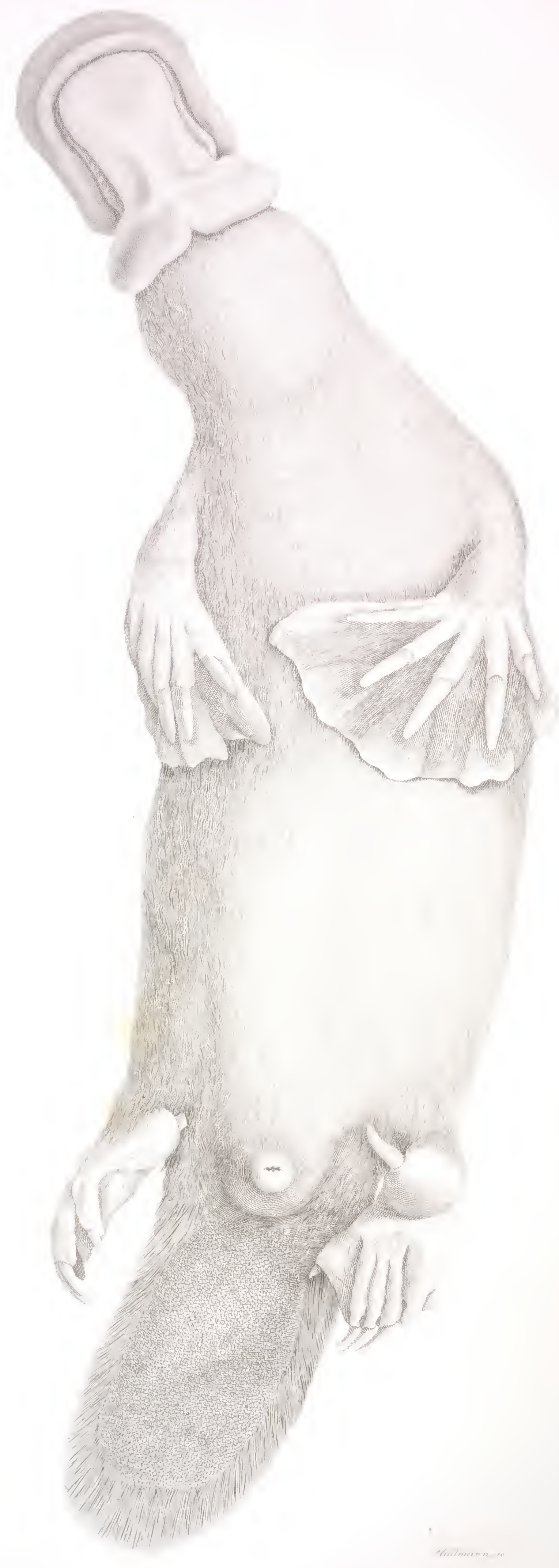




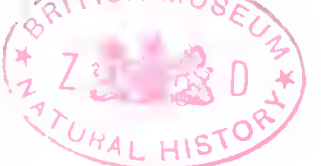




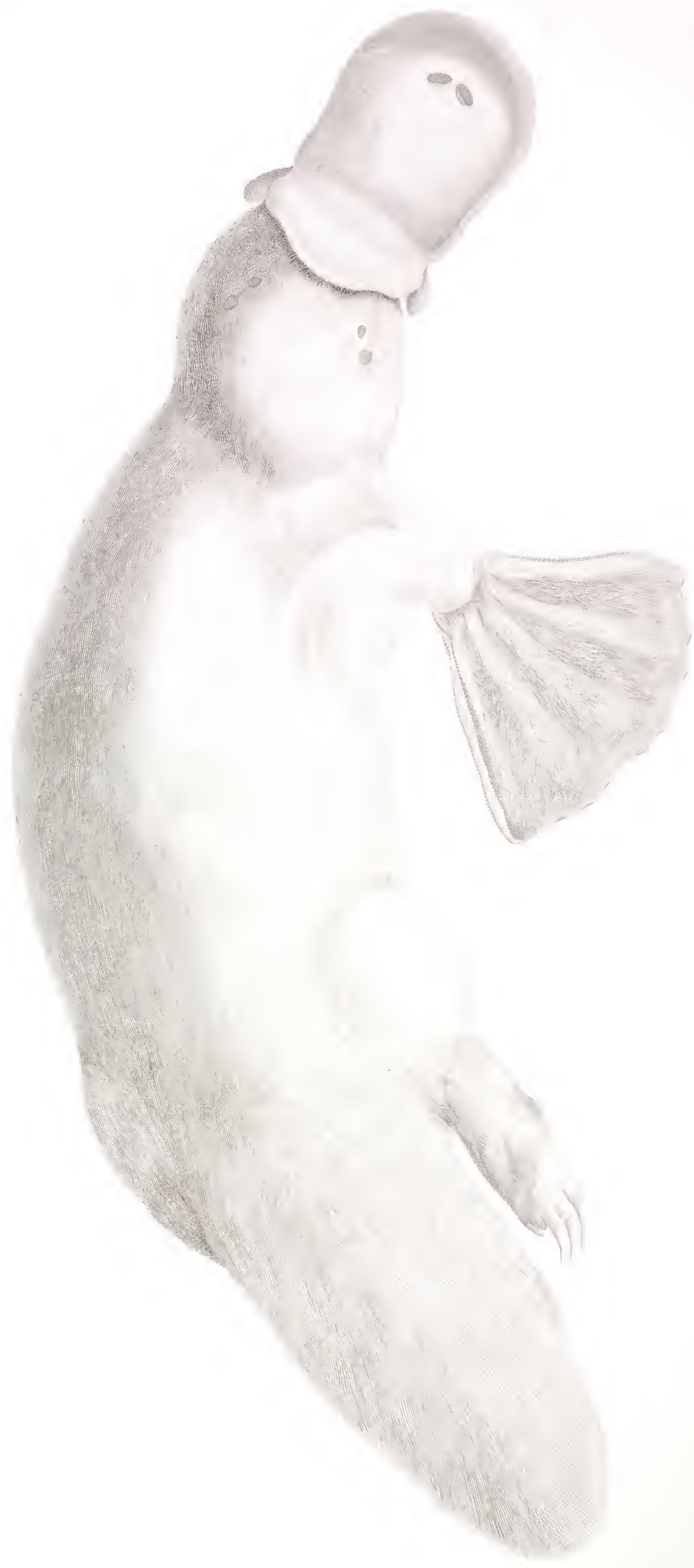




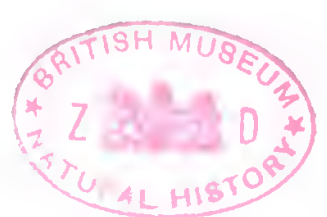





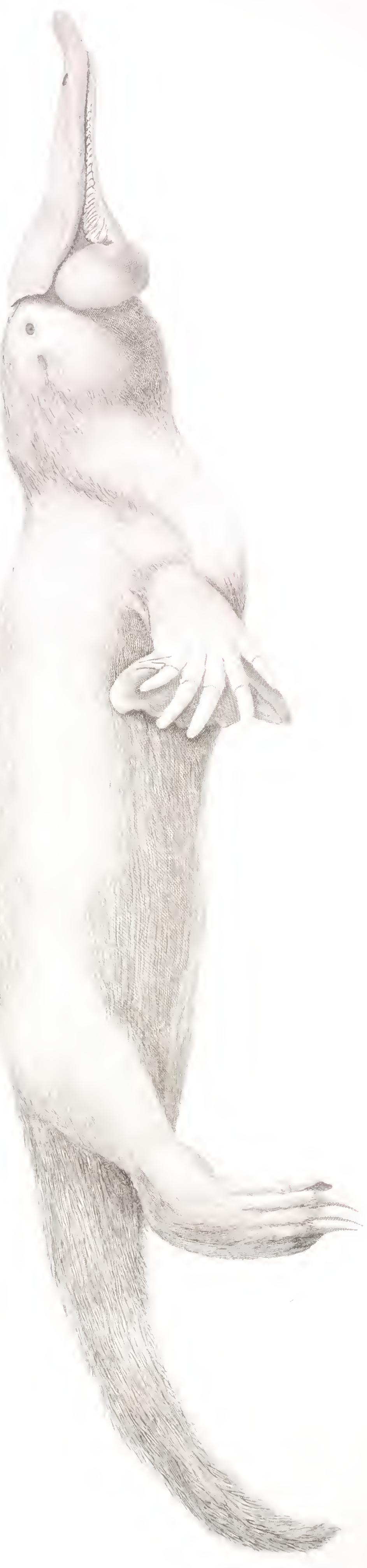




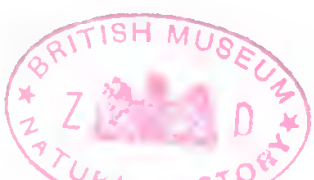

X 



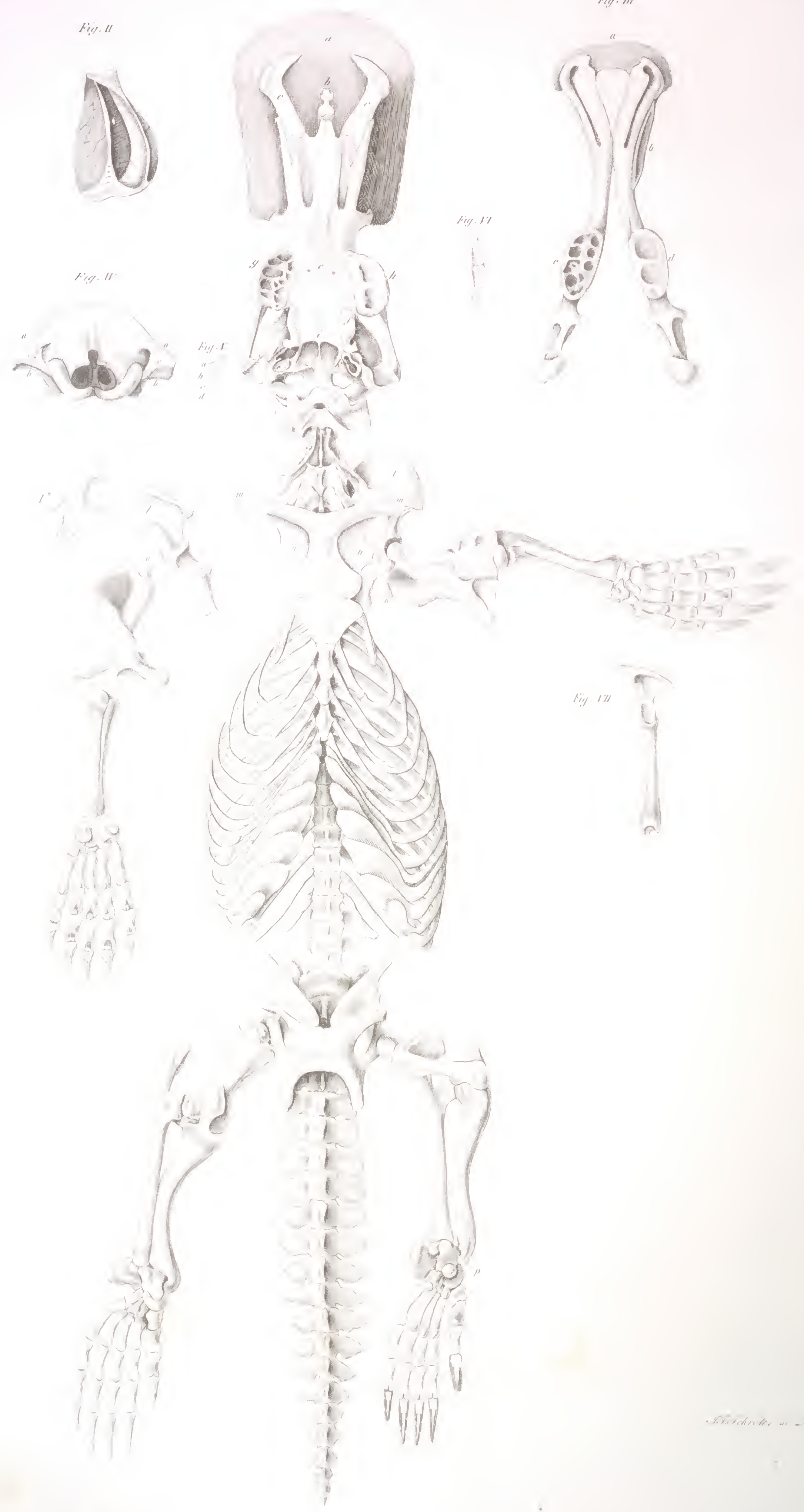




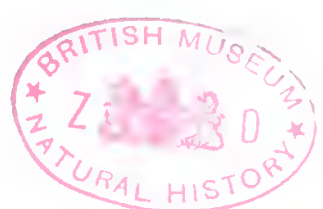






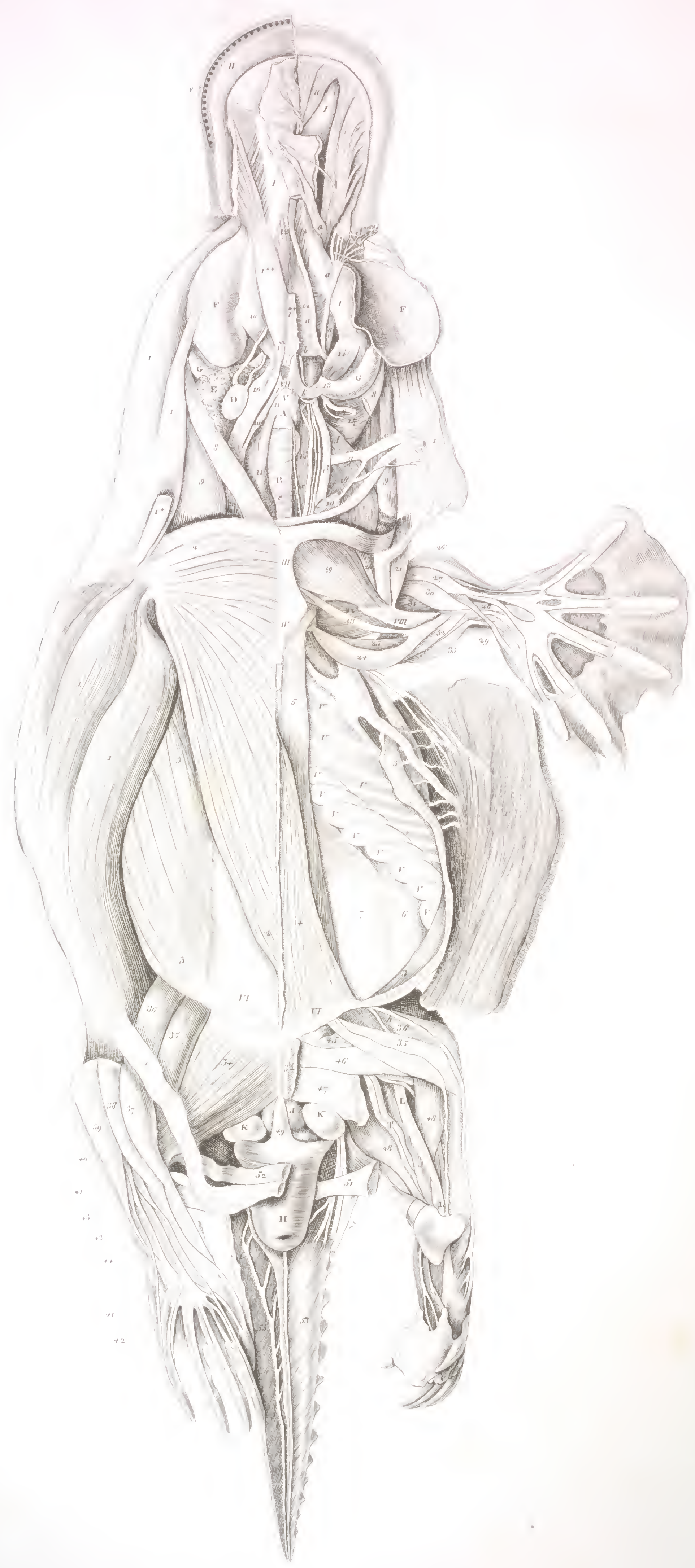




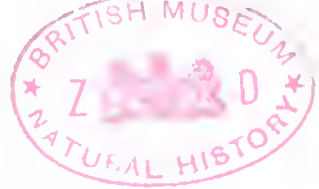





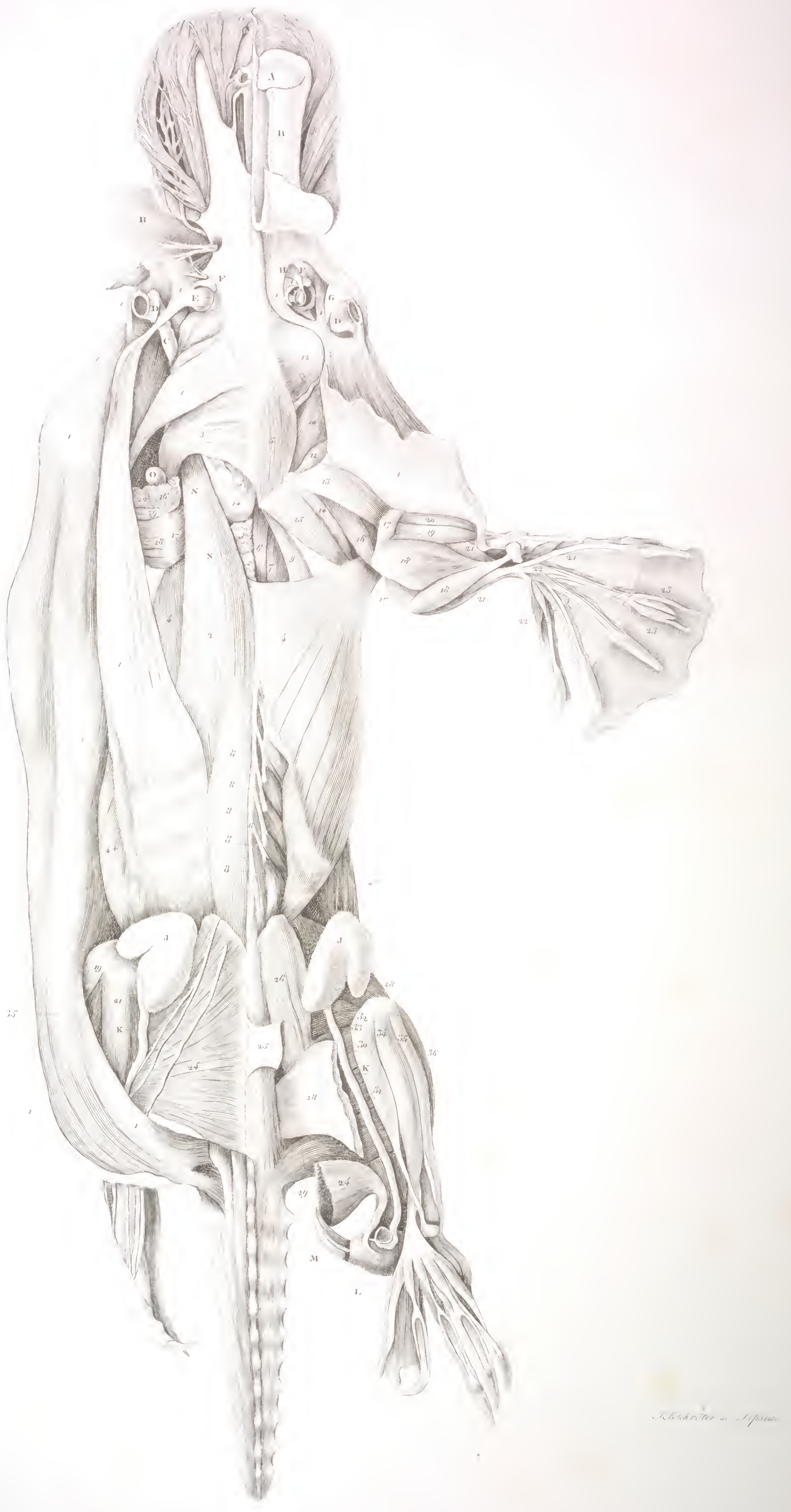





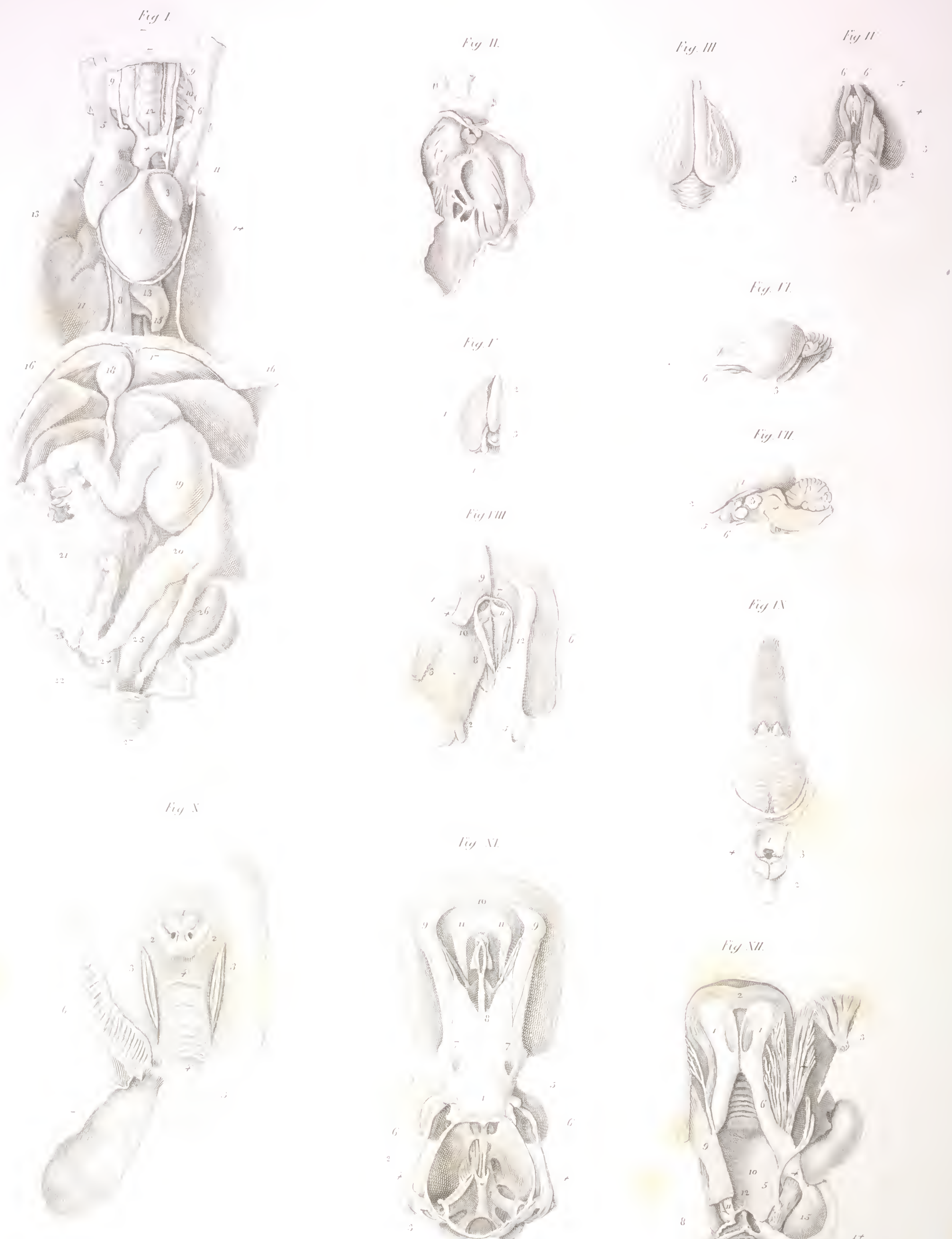
2 工 


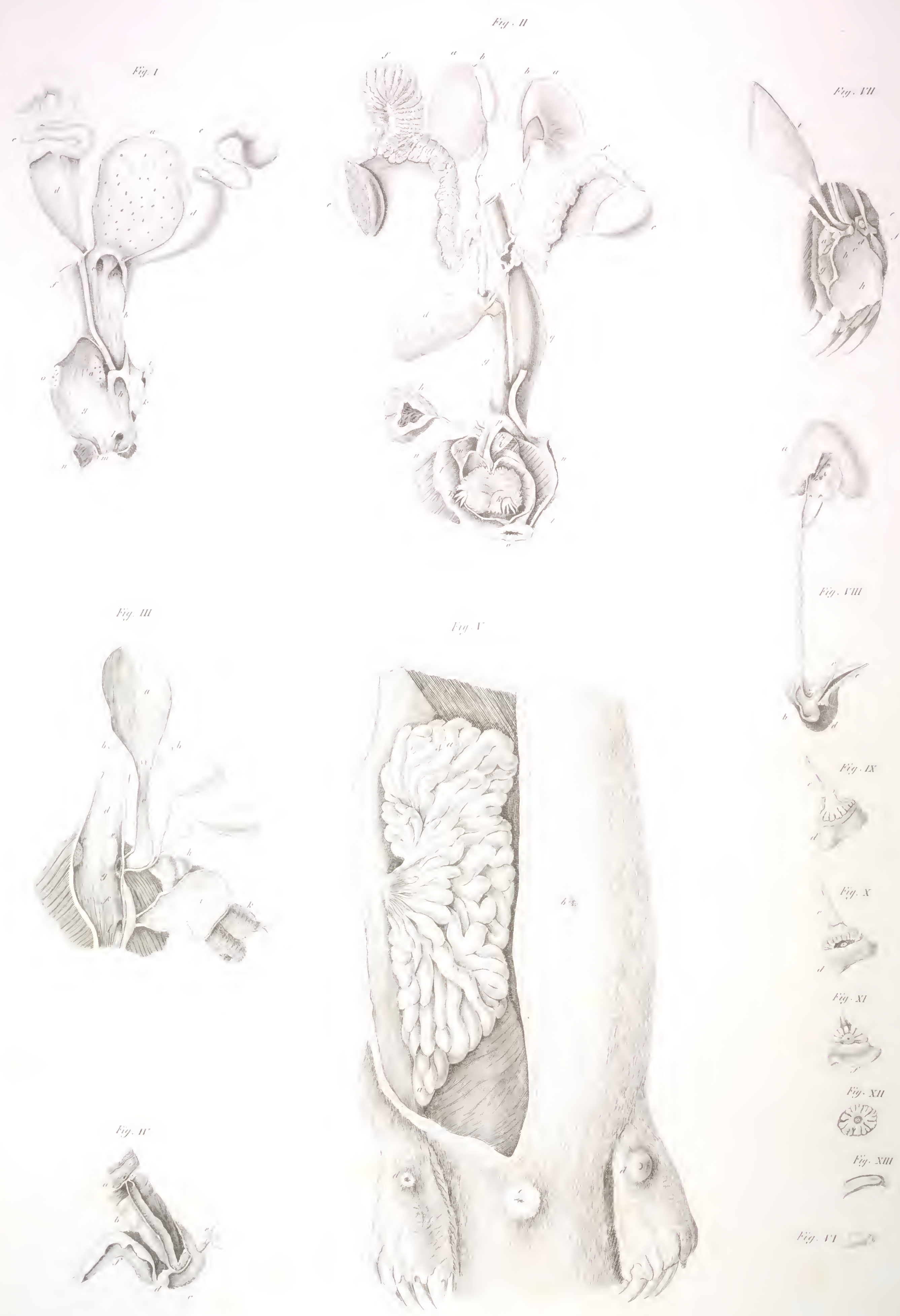


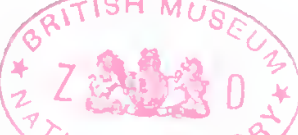

N 


